\title{
Import of Presequence-Containing \\ Precursor Proteins into Mitochondria
}

\author{
PhD Thesis \\ in partial fulfillment of the requirements \\ for the degree "Doctor of Philosophy (PhD)" \\ in the Molecular Biology Program \\ at the Georg August University Göttingen, \\ Faculty of Biology
}

submitted by

Jonathan Melin

born in

Memphis, Tennessee

United States of America 



\section{Thesis Committee Members}

(First Reviewer)

Prof. Dr. Peter Rehling

Institute for Cellular Biochemistry

University Medical Center, Göttingen

Göttingen, Germany

(Second Reviewer)

Prof. Dr. Ivo Feussner

Department of Plant Biochemistry

University of Göttingen

Göttingen, Germany

\section{Prof. Dr. Christian Griesinger}

Department of NMR-based Structural Biology

Max Planck Institute for Biophysical Chemistry

Göttingen, Germany 


\section{Affidavit:}

I herewith declare that this thesis has been written independently and with no other sources and aids than quoted.

Jonathan Melin

Göttingen, 21 May 2014 


\section{LIST OF PUBLICATIONS}

Data presented in this dissertation have been previously published or are in revision:

Melin, J., Schulz, C., Wrobel, L., Bernhard, O., Chacinska, A., Jahn, O., Schmidt, B., Rehling, P. (2014). Presequence recognition by the Tom40 channel promotes precursor translocation towards the inner membrane translocase. In revision.

Lytovchenko, O., Melin, J., Schulz, C., Kilisch, M., Hutu, D.P., and Rehling, P. (2013). Signal recognition initiates reorganization of the presequence translocase during protein import. EMBO J. 32, 886-898. 


\section{TABLE OF CONTENTS}

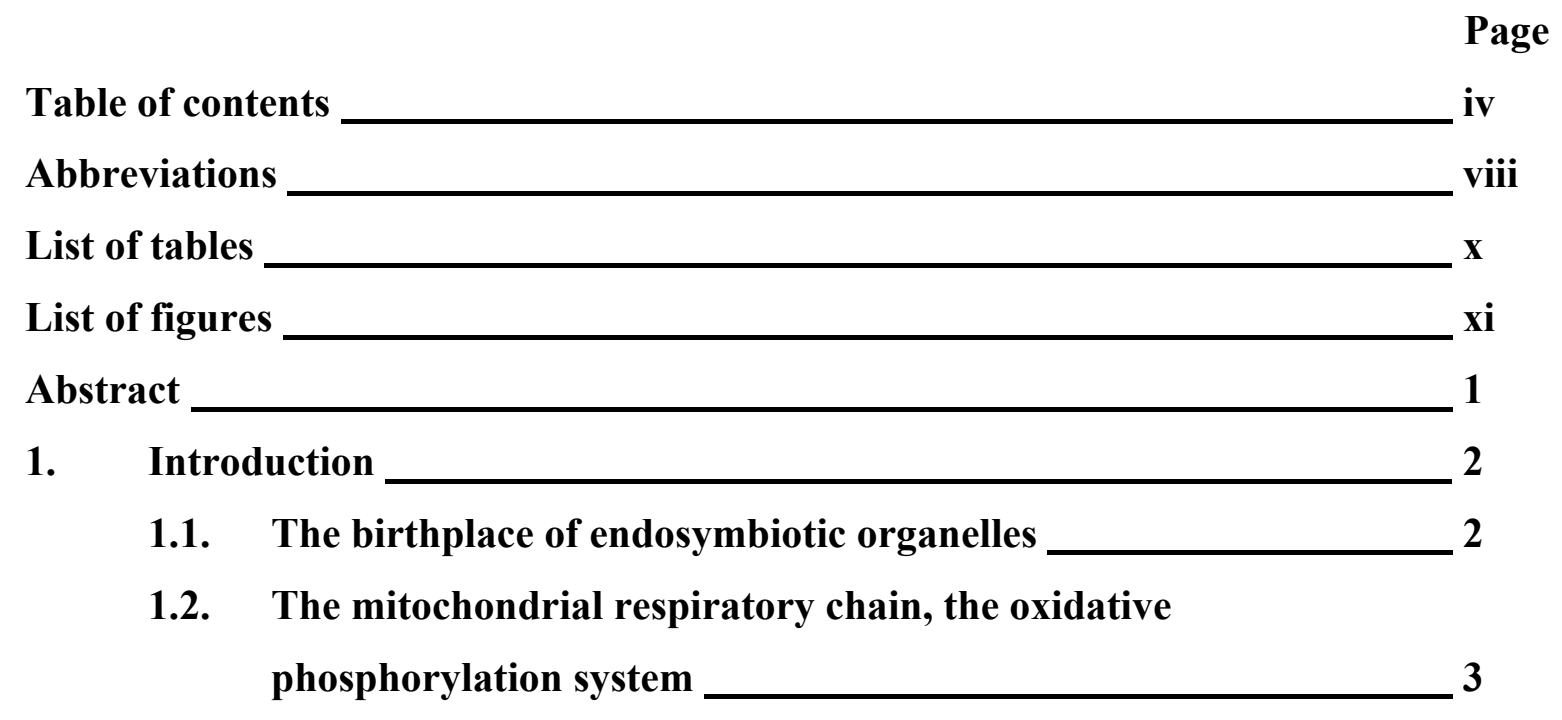

1.3. Mitochondrial protein import

1.3.1. Import of presequence-containing proteins, the presequence pathway 8

1.3.1.1. The presequence translocase associated motor, PAM 13

1.3.1.2. Presequence translocase dynamics, TIM23 $^{\text {MOTOR }}$ and TIM23 ${ }^{\text {SORT }}$ 14

1.3.1.3. Presequence import, the TOM-TIM23 supercomplex and TOM energetics 16

1.3.2. Mitochondrial import of metabolite carrier proteins, the carrier pathway 17

1.3.3. Mitochondrial import and assembly of outer membrane $\beta$-barrel proteins 20

1.3.4. Mitochondrial intermembrane space import and assembly pathway 22

1.3.5. Mitochondrial import of $\alpha$-helical outer membrane proteins 24

1.4. Research objectives 26

2. Materials and Methods 28

2.1. Materials 28

2.1.1. Chemicals 28

2.1.2. Consumables 29 
2.1.3. Kits 30

2.1.4. Enzymes _ 30

2.1.5. Peptides 31

2.1.6. Antibodies _ 31

2.1.7. Plasmids _ 32

2.1.8. Yeast Strains __ 33

2.1.9. Laboratory Equipment __ 34

2.1.10. Software 36

2.1.11. Buffers, Solutions and Media _ 36

2.2. E. coli manipulation _ـ 39

2.2.1. Preparation of chemically competent $E$. coli 39

2.2.2. Transformation of chemically competent $E$. coli _ 40

2.3. Molecular Biology 40

2.3.1. Plasmid isolation from XL-1 Blue E. coli _ 40

2.3.2. Polymerase chain reaction $(\mathrm{PCR}) \longrightarrow 40$

2.3.3. Agarose gel electrophoresis __ 41

2.3.4. Site-directed mutagenesis __ 41

2.3.5. Sequencing of DNA constructs

2.3.6. Peptide synthesis __ 42

2.4. S. cerevisiae methods _ 42

2.4.1. S. cerevisiae growth conditions _ 42

2.4.2. 5-FOA plasmid shuffling _ 43

2.4.3. Transformation of $S$. cerevisiae _ 43

2.4.4. Subcellular fraction of yeast, mitochondrial isolation __ 44

2.5. Purification of recombinant proteins from $E$. coli

2.5.1. BL21 E coli protein expression __ 44

2.5.2. Ion exchange chromatography _ـ 45

2.5.3. Metal affinity chromatography of recombinant proteins $\_45$

2.5.4. Size exclusion chromatography _ 46

2.6. PAGE protein analysis __ 46

2.6.1. SDS-PAGE

2.6.2. BN-PAGE 46

2.6.3. Coomassie Brilliant Blue staining _ 47

2.6.4. Colloidal Coomassie staining _ 47 
2.6.5. Western-blotting and immunolabeling of PVDF membrane

2.6.6. Digital autoradiography 48

2.6.7. Mass spectrometric analysis of proteins 48

2.7. In organello mitochondrial assays 49

2.7.1. In vitro transcription/translation and ${ }^{35} \mathrm{~S}$ labeling of proteins 49

2.7.2. In vitro import into isolated mitochondria 50

2.7.3. Mitochondrial membrane solubilization 50

2.7.4. In organello crosslinking 50

2.7.5. Immunoprecipitation 51

2.7.6. Metal affinity chromatography of the TOM complex 52

2.8. In vitro recombinant mitochondrial protein interaction assay 53

2.9. Homology modeling 54

3. Results - The presequence active Tom40 55

3.1. Tom40 is a major contributor to TOM presequence interaction $\_55$

3.2. Tom40 displays two discrete presequence-interacting regions 59

3.3. TOM complex stability is unaltered in Tom40 phosphomimetic (T220E) and phosphoblock (T220V) mutants 65

3.4. Import analysis of Tom 40 phospho-mutants 67

3.5. Functional characterization of TOM translocation intermediates within Tom40 phospho-mutants 69

3.6. Investigating the functional relationship between the presequence-binding site of Tom40 and Tom $22^{\mathrm{IMS}}$ 71

3.7. Tom40 phosphorylation at $\mathbf{T 2 2 0}$ allows for the manipulation of import kinetics along the presequence pathway 74

3.8. The characterization of a novel TIM23 interaction between Tim50 and Tim21 77

3.9. Presequences trigger the dissociation of Tim50 from Tim 21 and prime the presequence translocase for translocation 79

4. Discussion 84

4.1. The Tom40 channel recognizes presequences and augments their translocation kinetics in route to the inner membrane 84

4.2. Presequence association at the TOM complex, Tom40's role 85 
4.3. Regulation of mitochondrial import via posttranslational modification at the TOM complex

4.4. Tom40's roles within the context of the posttranslationally mediated TOM import regulatory system 88

4.5. The dissociation of the Tim23 coordinated Tim21-Tim50 interaction is functionally linked to presequence translocase priming 90

4.6. A presequence priming model of matrix import by the presequence translocase 91

4.7. TIM23 dynamics _ 93

5. Summary and future perspectives _ 96

References 99

Acknowledgments 119

Curriculum Vitae 120 


\section{ABBREVIATIONS}

ATP

BN

BSA

${ }^{\circ} \mathrm{C}$

CSM

DMP

DNA

dNTP

DTT

ECL

EDTA

$\mathrm{FADH}_{2}$

hr

HCl

HEPES

HRP

IgG

IM

IMS

IP

IPTG

kDa

$\min$

MOPS

MPP

NADH

NMR

OD

OM

PAGE

PAM
Adenosine triphosphate

Blue native

Bovine serum albumen

Degree Celsius

Complete supplement mixture

Dimethyl pimelimidate

Deoxyribonucleic acid

2'-deoxynucleoside-5'-triphosphate

Dithiothreitol

Enhanced chemiluminescence

Ethylenediaminetetraacetic acid

Flavin adenine dinucleotide

Hour(s)

Hydrochloric acid

2-[4-(2-hydroxyethyl)piperazin-1-yl]ethanesulfonic acid

Horseradish peroxidase

Immunoglobulin $\mathrm{G}$

Inner membrane

Inner membrane space

Immunoprecipitation

Isopropyl $\beta$-D-1-thiogalactopyranoside

Kilodaltons

Minute(s)

3-morpholinopropane-1-sulfonic acid

mitochondrial processing peptidase

Nicotinamide adenine dinucleotide

Nuclear magnetic resonance

Optical density

Outer membrane

Polyacrylamide gel electrophoresis

Presequence translocase associated motor 
PCR

PMF

PMSF

ProtA

PVDF

RNA

ROS

rpm

SAM

SA-HRP

SDS

sec

SEM

SEM buffer

TAE

TBS

TCA

TIM

TIM22

TIM23

TOM

Tris

UV

$\Delta \psi$
Polymerase chain reaction

Proton motive force

Phenylmethylsulfonyl fluoride

Protein A

Polyvinylidene fluoride

Ribonucleic acid

Reactive oxygen species

Revolutions per minute

Sorting and assembly machinery of the outer membrane

Streptavidin horseradish peroxidase

Sodium dodecyl sulfate

Second(s)

Standard error of the mean

Sucrose EDTA MOPS buffer

Tris acetate EDTA

Tris buffered saline

Trichloroacetic acid

Translocase of the inner membrane

Carrier translocase of the inner membrane

Presequence translocase of the inner membrane

Translocase of the outer membrane

2-Amino-2-hydroxymethyl-propane-1,3-diol

Ultraviolet

Membrane potential 


\section{LIST OF TABLES}

Page

Table 1: List of non-standard chemicals and their respective producers 28

Table 2: List of consumables, together with producers 29

Table 3: List of kits and suppliers 30

Table 4: List of enzymes and manufactures 30

Table 5: List of peptides, sequences and producers/literature reference 31

Table 6: List of primary antibodies and corresponding epitopes 31

Table 7: List of plasmids 32

Table 8: List of yeast strains 33

Table 9: List of laboratory equipment, along with the corresponding suppler 34

Table 10: List of software, along with the corresponding use and developer 36

Table 11: List of buffers and solutions 37 


\section{LIST OF FIGURES}

Page

Figure 1: A schematic representation of the mitochondrial respiratory chain 5

Figure 2: Mitochondrial targeting signals and corresponding import and sorting routes 7

Figure 3: The mitochondrial presequence pathway 8

Figure 4: The mitochondrial carrier pathway 18

Figure 5: The mitochondrial sorting and assembly (SAM) pathway 21

Figure 6: The mitochondrial intermembrane space import and assembly (MIA) pathway 23

Figure 7: The import of mitochondrial $\alpha$-helical outer membrane (OM) proteins 25

Figure 8: A schematic representation of the presequence probes 55

Figure 9: In organello photo-crosslinking in isolated wild-type mitochondria 56

Figure 10: Immunoisolation of the TOM complex via Tom 22 antiserum from digitonin-solubilized wild-type mitochondria, previously subjected to in organello photo-crosslinking 57

Figure 11: In organello photo-crosslinking in mutant mitochondria 58

Figure 12: Workflow of joint photo-crosslinking/mass spectrometry approach to Tom40 presequence interaction mapping 59

Figure 13: Ni-NTA isolation of the TOM complex from digitonin-solubilized Tom $22^{\text {HIS }}$ mitochondria, followed by in vitro TOM complex photo-crosslinking 60

Figure 14: Large-scale Ni-NTA isolation of the TOM complex from digitonin-solubilized Tom $22^{\mathrm{HIS}}$ mitochondria, followed by in vitro TOM complex photo-crosslinking 61

Figure 15: Fragment ion mass spectra of presequence-probe crosslinked Tom40 peptides 62

Figure 16: Tom 40 homology model based upon the mouse VDAC structure 64

Figure 17: $\operatorname{tom} 40^{E m u t}$ and tom $40^{V m u t}$ alleles display no growth phenotype and wild-type-like TOM stability 66

Figure 18: Tom40 phospho-mutants exhibit wild-type-like mitochondrial protein levels and TOM stability 67

Figure 19: Photo-crosslinking in isolated Tom $40^{\mathrm{E}}$ and $\operatorname{Tom} 40^{\mathrm{V}}$ mitochondria 68 
Figure 20: Carrier and MIA pathway import analysis in Tom40 phospho-mutant mitochondria

Figure 21: Presequence mediated TOM translocation analysis in Tom40 phospho-mutant mitochondria 70

Figure 22: $\operatorname{tom} 40^{E m u t}$ and tom $40^{V m u t}$ alleles display unaffected growth, protein levels and TOM stability in the tom22-2 background 72

Figure 23: $\operatorname{tom} 40^{E m u t}$ and tom $40^{V m u t}$ alleles equally exhibit reduced TOM presequence translocation in the tom 22-2 background 73

Figure 24: $\operatorname{Tom} 40^{\mathrm{E}}$ and $\operatorname{Tom} 40^{\mathrm{V}}$ mitochondria exhibit opposing alterations in presequence mediated matrix import kinetics 75

Figure 25: $\operatorname{Tom} 40^{\mathrm{E}}$ and $\operatorname{Tom} 40^{\mathrm{V}}$ mitochondria display sensitivity to the loss of Tom $22^{\mathrm{IMS}}$ in presequence mediated matrix import 76

Figure 26: $\operatorname{Tim} 21$ can be crosslinked to $\operatorname{Tim} 50$ in a presequence sensitive manner _ 78

Figure 27: $\operatorname{Tim} 23^{\mathrm{IMS}}$ establishes the Tim21-Tim50 interaction 79

Figure 28: Tim 50 and Tim 21 dissociate upon signal sequence binding at the presequence translocase, leading to Pam17 association

Figure 29: Tim50's presequence-binding domain is dispensable in the presequence-triggered dissociation of Tim 21 from Tim50 82

Figure 30: Schematic representation of presequence-passage through Tom 40 of the TOM complex 89

Figure 31: Schematic representation of the dynamic stages of the presequence translocase (TIM23) during matrix import 92 


\section{ABSTRACT}

The presequence pathway is utilized by over $70 \%$ of all cytosolically translated proteins destined to the mitochondria, emphasizing the significance imparted by presequence import on mitochondria translocation. Presequence docking at the main mitochondrial entry gate, the translocase of the outer mitochondrial membrane (TOM), has been the target of a vast number of publications throughout the past two and a half decades. However, the dynamic docking events leading up to the engagement of the presequence with the TIM23 complex (also known as the presequence translocase) is still poorly understood.

This study presents Tom40 as a presequence-active TOM complex subunit, and maps its presequence-interacting sites employing presequence probes in a joint photocrosslinking/mass spectrometry based approach. Moreover, the interacting regions of Tom40 were placed within a recently published, biochemically grounded Tom40 homology model, where two presequence-active regions were identified. A posttranslationally active residue was identified within one of the defined presequenceinteracting surfaces. Phospho-mimetic and phospho-block Tom40 point mutants were shown to selectively augment local presequence import kinetics at the TOM complex, resulting in alteration of overall import kinetics. Finally, the established intermembrane space (IMS) domain of Tom 22 was functionally linked to Tom40 presequence interaction.

Examining the following stages of presequence import, this study presents a series of dynamic subunit exchange events leading to presequence presentation at the channel of the presequence translocase. A novel presequence translocase interaction between Tim50 and Tim 21 was characterized and shown in vitro to be coordinated by the IMS domain of Tim23 via a Tim50 interaction mechanism. Moreover, the interaction of Tim50 and Tim21 was shown in organello to be signal sequence sensitive, as Tim21 is demonstrated to dissociate from Tim50 within the presequence translocase in a presequence-"priming" dependent manner. Finally, the aforementioned presequence translocase priming event is linked to matrix translocation as the early presequence associated motor (PAM) subunit Pam17 was shown to be recruited to the TIM23 complex concomitantly with Tim 21 dissociation during TIM23 priming.

Taken together, these data shine new light on the presently elusive transfer mechanism of presequence-containing substrates from the outer membrane resident TOM channel to the inner membrane bound TIM23 complex. 


\section{INTRODUCTION}

\subsection{The birthplace of endosymbiotic organelles}

The eukaryotic cell is set apart from its prokaryotic counterpart by the presence of intracellular membrane-enclosed organelles (Palade, 1964). Virtually all of these organelles are part of the characteristic endomembrane system, which facilitates the partitioning of the cell interior, allowing for the segregation of various biochemical processes. Mitochondria and plastids are the only omissions to this intracellular membrane system due to their non-host origin. These evolutionarily distinct organelles arose through systematic endosymbiotic events with the conception of mitochondria preceding that of the plastids (Gross and Bhattacharya, 2009). In the case of the mitochondrion, strong evidence exists illustrating the occurrence of an endosymbiotic event in which a Gram-negative purple non-sulfur bacteria ( $\alpha$-proteobacteria) was phagocytosed by a protoeukaryote host cell (Gray et al., 1999). Following engulfment, the $\alpha$-proteobacteria likely escaped from the food vacuole and was free to incorporate fitness-conferring host cell proteins through protomitochondrial resident outer membrane (OM) proteins (Cavalier-Smith, 2006). This endosymbiotic event is believed to have occurred approximately two billion years ago (Bhattacharya et al., 2007; de Duve, 2007; Dolezal et al., 2006; Dyall, 2004). Following the initial engulfment event, endosymbiont resident genes were transferred to the host genome through four predominant gene transference mechanisms; endosymbiotic gene transfer, de novo gene construction, horizontal gene transfer and co-option of existent host functions (Gross and Bhattacharya, 2009). It has been suggested on numerous occurrences that the selection pressure for the migration of protomitochondrial genes to the nucleus was due to advantages conferred to the host regarding $\alpha$-proteobacterial energy production via oxidative phosphorylation (Andersson et al., 2003; Kurland and Andersson, 2000). Therefore, fitness-conferring novel gene transference events leading to increased energy production efficiency rewrote the protomitochondrial genome. This process resulted in the shrinking of its size from an estimated 603 distinct genes (Gabaldón and Huynen, 2003) to the mitochondrial genome as we know it today, encoding 8 proteins in yeast (S. cerevisiae) and 13 proteins in humans. The retention of these select few mitochondria-encoded genes demanded the preservation of a complete array of transcriptional and translational systems. Importantly, this mandated the coevolution of a highly sophisticated network of dynamic import translocases, competent in the concomitant intraorganellar sorting of an enormous 
number of nuclear-encoded protein and RNA substituents within the mitochondrion. This apparent energetics conundrum, in which several orders of magnitude more anabolically active biomolecules must be transcribed and translated outside of the organelle and subsequently imported to give rise to the seemingly insignificant number of mitochondriaencoded genes, has baffled researchers for nearly a century.

Succinctly, evolution has betrothed these endosymbiotic organelles with a vital and unique set of functions (energy production, amino acid and lipid metabolism, biosynthesis of Fe-S clusters and apoptosis) required by all forms of eukaryotic life, thereby instilling an essential metabolic role upon these extraordinary organelles.

\subsection{The mitochondrial respiratory chain, the oxidative phosphorylation system}

Arguably the most recognized metabolic function of the mitochondrion is the production of energy-rich biologically active compounds by way of oxidative phosphorylation (OXPHOS) to meet the cellular energy demands. OXPHOS mediated energy production in the mitochondria can be separated into two major events: (i) the production of a proton based electrochemical gradient (membrane potential $[\Delta \psi]$ ) across the inner mitochondrial membrane through the enzymatic oxidation of reducing equivalents (NADH and $\mathrm{FADH}_{2}$ ) obtained from central catabolic metabolic processes, e.g. the tricarboxylic acid cycle, (ii) the utilization of the $\Delta \psi$ to drive the production of the stable high energy molecule, ATP.

The first of the aforementioned steps is performed by the respiratory chain complexes of the mitochondrial inner membrane (IM) (Fig. 1). In S. cerevisiae, the respiratory chain contains three complexes which mediate the oxidation of reducing equivalents, these being succinate dehydrogenase (II), the cytochrome $b c_{1}$ complex (III) and the cytochrome $c$ oxidase complex (IV), and two electron-shuttling entities (coenzyme Q and cytochrome $c$ ) (Stuart, 2008) (Fig. 1A). Moreover, in yeast, no true complex I exists and subsequently the oxidation of NADH is carried out by three membrane-bound proteins, Nde1, Nde2 and Ndj1. In yeast, complexes III and IV serve to produce the proton motive force (PMF), which is utilized by the $\mathrm{F}_{1} \mathrm{~F}_{\mathrm{o}}$-ATP synthase (also called complex V) (Fig. 1A). Additionally, the S. cerevisiae mitochondrial genome encodes for eight proteins, seven core catalytic subunits of the respiratory chain and one component of the mitochondrial ribosome (Lipinski et al., 2010) (Fig. 1A). 
The OXPHOS situation in the human mitochondrial IM is slightly different from yeast, with the respiratory chain containing a true NADH dehydrogenase (complex I), which in combination with complex III and IV contribute to the $\Delta \psi$ across the IM (Papa et al., 2012) (Fig. 1B). Another major discrepancy from yeast seen in the human respiratory chain is the number of mitochondria-encoded respiratory chain subunits. The human mitochondrial genome encodes 13 different proteins, all of which are compounds of the respiratory chain, with a majority being key subunits of the NADH dehydrogenase, complex I.

Refuting the original conception that the mitochondrial respiratory chain complexes were randomly distributed in the IM (the fluid model) (Hackenbrock et al., 1986; Hatefi, 1985), the discovery of the mild non-ionic detergent digitonin in conjunction with the establishment of blue native polyacrylamide gel electrophoresis (BN-PAGE) shook up the field (Cruciat et al., 2000; Schägger and Pfeiffer, 2000). In these key early publications, it was shown that the respiratory chain is organized in higher order supercomplexes, and the concept of the mitochondrial respirasome was introduced. Moreover, later studies utilized negative stain or cryo-electron microscopy to image detergent solubilized higher order respiratory chain structures (referred to as respirasomes) (Althoff et al., 2011; Dudkina et al., 2011; Schäfer et al., 2006). Today, the existence of respirasomes is widely accepted and known to contain complexes I (in higher eukaryotes), III and IV (Winge, 2012). However, it should be noted that there are a few skeptics in the field (Barrientos and Ugalde, 2013). Recent findings have provided genetic evidence for respiratory chain supercomplexes, as respirasome dynamics were shown to dictate the electron flux in response to different substrates (Lapuente-Brun et al., 2013). 

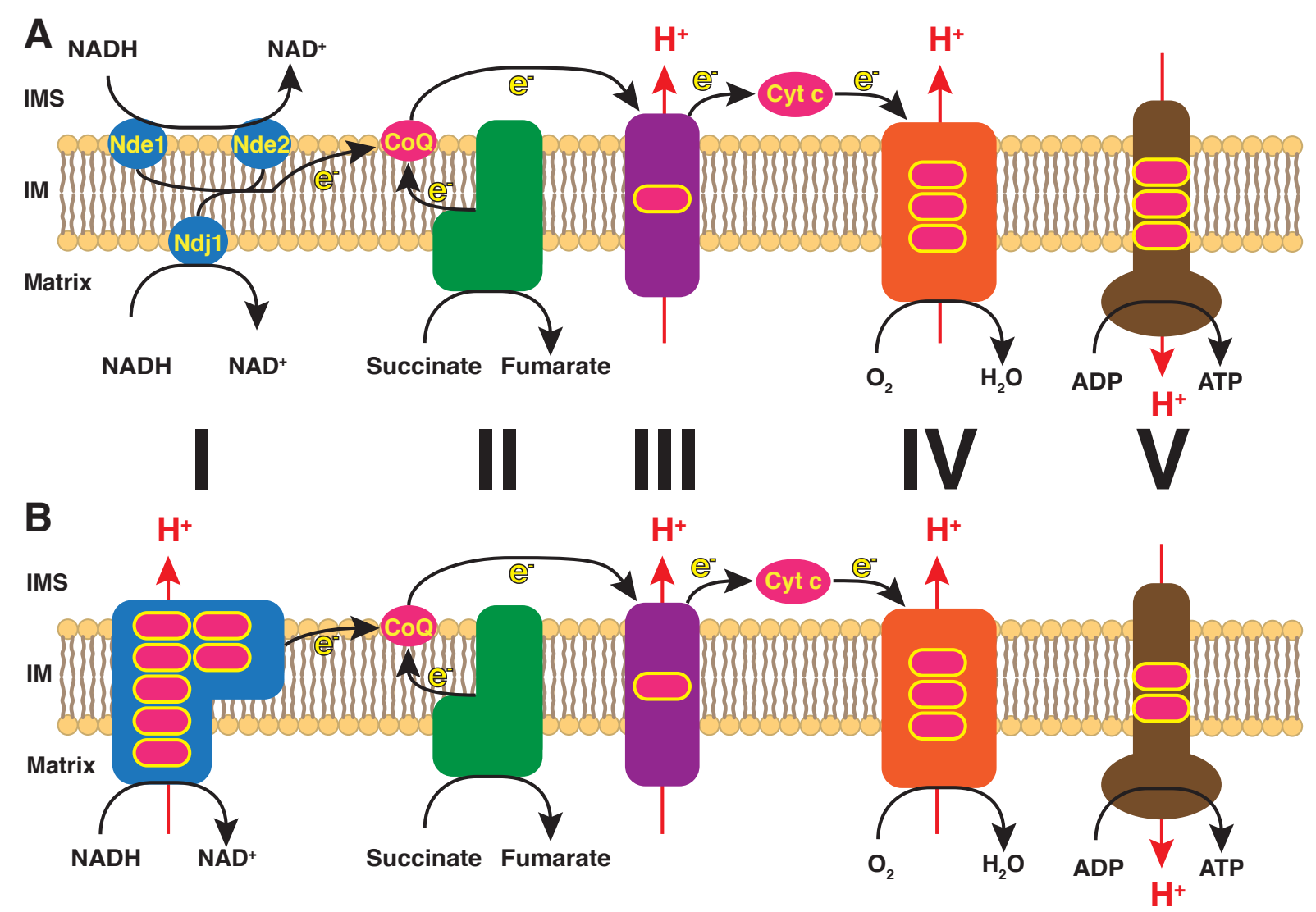

Figure 1. A schematic representation of the mitochondrial respiratory chain, illustrating the electron flux through the individual respiratory complexes. Red arrows indicate the flow of protons throughout the respiratory chain. (A) Cartoon depiction of the $S$. cerevisiae respiratory chain consisting of complexes II - V with all of the mitochondria-encoded subunits in pink with a yellow outline. (B) Cartoon depiction of the human respiratory chain consisting of complexes I - V with all of the mitochondria-encoded subunits in pink with a yellow outline. Complex I (human only) NADH dehydrogenase, complex II - succinate dehydrogenase, complex III - cytochrome $b c_{l}$ complex, complex IV - cytochrome $c$ oxidase and complex V - $\mathrm{F}_{1} \mathrm{~F}_{\mathrm{o}}-\mathrm{ATP}$ synthase. $\mathrm{e}^{-}$, electron. CoQ, coenzyme Q. Cyt c, cytochrome $c$. IMS, intermembrane space. IM, inner membrane.

\subsection{Mitochondrial protein import}

Over $99 \%$ of all mitochondrial resident proteins are nuclear-encoded, translated on cytosolic ribosomes and posttranslationally imported into one of the organelle's four subcompartments (Pfanner et al., 2004). Due to the multitude of different intraorganellar targeting destinations in conjunction with highly divergent substrate characteristics, a set 
of unifying mitochondrial-targeting signals has evolved (Chacinska et al., 2009). These signals have been divided into two major classes, these being the well-defined Nterminally located mitochondrial presequence (Fig. 2A) and the highly heterogeneous class of internal-targeting signals (Fig. 2B - E). The class of internal-targeting signals consists of all mitochondrial-orienting signals that cannot be characterized as a presequence. In brief, these signals are subclassified as the IM targeting signal of the carrier pathway (Ryan et al., 1999; Wagner et al., 2008; Wiedemann et al., 2001), the C-terminally located $\beta$ signal (present in OM $\beta$-barrel proteins) (Kutik et al., 2008), the intermembrane space (IMS) directing cysteine-containing signals of the mitochondrial intermembrane space import and assembly (MIA) pathway (Gabriel et al., 2007; Milenkovic et al., 2007; 2009) and the $\mathrm{N}$ - or $\mathrm{C}$-terminal (signal or tail anchor sequence, respectively) $\alpha$-helical type present in $\alpha$-helical OM proteins (Becker et al., 2011; Papic et al., 2011; Setoguchi et al., 2006; Stojanovski et al., 2007). 


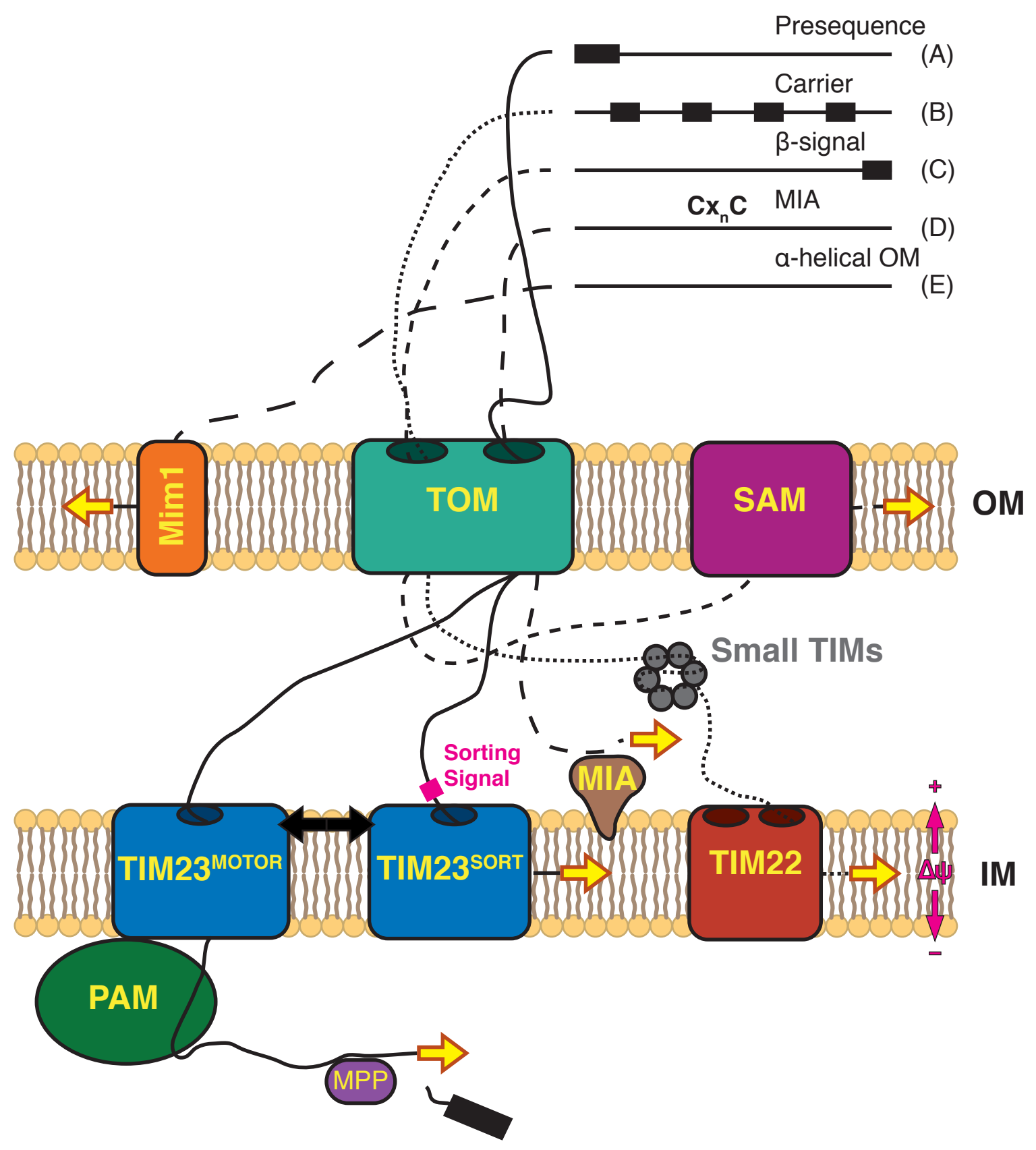

Figure 2. Mitochondrial targeting signals and corresponding import and sorting routes. (A) Presequence-containing substrate enters the mitochondrion via the translocase of the outer membrane (TOM). At the inner membrane (IM), presequence-containing substrates interact with the presequence translocase (TIM23) in either the SORT (sorting signal containing substrates) or MOTOR form, which contains the presequence associated motor (PAM). Matrix processing peptidase, MPP. (B) Substrates of the carrier pathway enter the mitochondrion through TOM and interact with small TIMs after entering the intermembrane space (IMS), shuttling the substrate to the TIM22 complex. (C) $\beta$ signal containing substrates ( $\beta$-barrel proteins of the outer membrane $[\mathrm{OM}])$ are imported first via TOM and then handed off to the sorting and assembly machinery (SAM). (D) $\mathrm{Cx}_{\mathrm{n}} \mathrm{C}$ containing substrates cross the OM at TOM and follow the mitochondrial intermembrane space import and 
assembly (MIA) pathway. (E) Single and multiple transmembrane domain containing $\alpha$-helical OM proteins are integrated into the OM via the Mim1 complex.

\subsubsection{Import of presequence-containing proteins, the presequence pathway}

Presequences are characterized as N-terminally located, net positively charged, amphipathic $\alpha$-helices. Mass spectrometry based analyses have estimated that approximately $70 \%$ of mitochondrial substrates contain cleavable presequences (Vögtle et al., 2009), however, many noncleavable presequence-containing substrates have already been characterized, significantly increasing the size of the presequence-containing substrate class. Therefore, the presequence pathway represents the single most important mitochondrial entry route. The presequence pathway allows for substrates to be fully imported into the mitochondrial matrix or for a single transmembrane span to be inserted into the mitochondrial IM (Fig. 3). Presequence mediated import commences at the main mitochondrial entry gate, TOM complex.

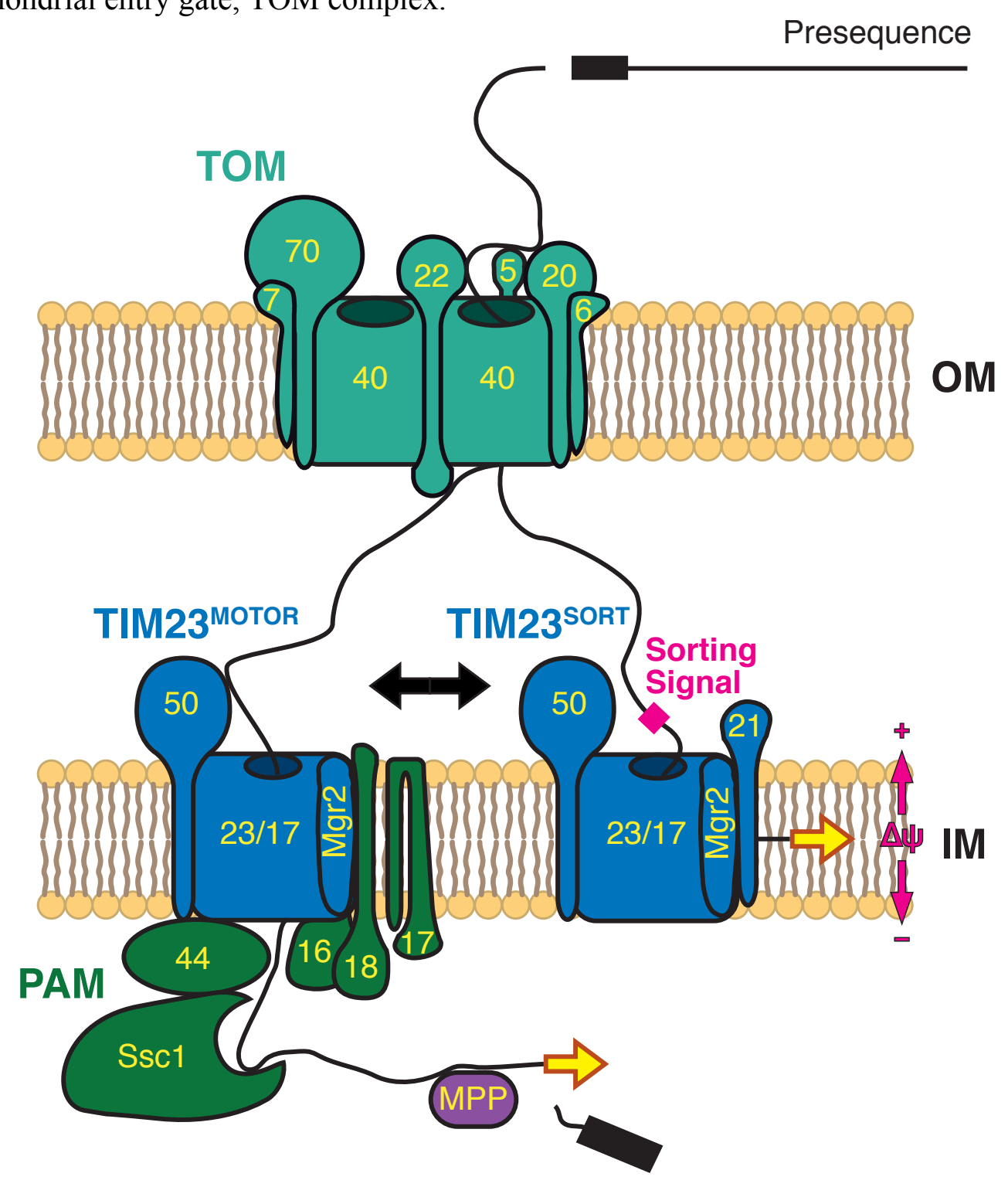


Figure 3. The mitochondrial presequence pathway. (A) Presequence-containing substrate entering the mitochondrion via the translocase of the outer membrane (TOM), functionally interacting with Tom20, Tom22, Tom5 and Tom40. At the inner membrane (IM), presequence-containing substrates interact with the presequence translocase (TIM23) in either the SORT (sorting signal containing substrates) or MOTOR form for translocation of single transmembrane domain containing precursors into the inner membrane or matrix translocation utilizing the presequence associated motor (PAM) followed by potential presequence removal by the matrix processing peptidase (MPP), respectively. OM, outer membrane.

The TOM complex is the main entry gate into the mitochondrion, translocating all mitochondrial substrates with the exception of several OM integrated proteins (see section 1.3.5. for details) (Endo and Yamano, 2010). Cryo-EM analysis has resolved the core structure to a resolution of $18 \AA$ and $3 \mathrm{D}$ reconstruction produced a near three-fold symmetrical complex with three pores (each with the dimensions $15 \AA$ x $25 \AA$ ) on one side which converge to a single cavity on the opposite side (Model et al., 2008). The core of the TOM complex is extremely stable (Meisinger et al., 2001) and comprised of the essential Tom40, the $\beta$-barrel pore-forming subunit (Becker et al., 2005; Hill et al., 1998; Suzuki et al., 2004), and Tom22, the central presequence receptor which exposes presequence recognition domains to both the cytoplasm as well as the IMS (Brix et al., 1997; Moczko et al., 1997). Additional subunits of the TOM complex are Tom70 (serves as a receptor for hydrophobic substrates such as carrier and ß-signal substrates [see section 1.3.2. and 1.3.3. for details, respectively]), Tom20 (the initial presequence receptor [Saitoh et al., 2007]), Tom71 (a low abundant Tom70 homolog [Schlossmann et al., 1996]), Tom 5 (a TOM assembly factor and presequence receptor [Dietmeier et al., 1997]), Tom6 (a TOM assembly factor [Kassenbrock et al., 1993]) and Tom7 (a TOM disassembly factor involved in TOM dynamics [Hönlinger et al., 1996]).

Mitochondrial association with presequence-containing substrates commences with their interaction with Tom20 (Saitoh et al., 2007). The obtainment of structural data of Tom20 in complex with a presequence has imparted the field of mitochondrial import with a wealth of data, as it represents the first published structurally based receptor presequence interaction information (Abe et al., 2000). This study confirmed the suspected hydrophobic mode of interaction between the presequence and the Tom 20 receptor domain (Brix et al., 1997). Following the interaction with Tom20, the presequence is transferred to the cytosolic receptor domain of the central receptor of the TOM complex, Tom22 (van Wilpe et al., 1999). The interaction of the presequence with Tom 22 has been shown to be 
mediated by ionic forces (Brix et al., 1997), leading to the conception of the possible simultaneous binding, tertiary complex formation, of the presequence by both Tom 20 and Tom22 (Shiota et al., 2011; Yamano et al., 2008). After the presequence has reached the central receptor, it is passed to Tom40, with the assistance of the small protease resistant TOM receptor, Tom5 (Dietmeier et al., 1997). At this point, the presequence is believed to be passed through the Tom 40 pore and passed to the trans-binding site of the TOM complex, the presequence-binding IMS domain of Tom22 (Komiya et al., 1998; Moczko et al., 1997; Shiota et al., 2011). The IMS domain of Tom22 has been shown to play an important presequence-anchoring role under suboptimal import conditions such as increased ionic stress, a reduction in $\Delta \psi$, the addition of a tightly folded substrate on the cis side of the TOM complex or the protease mediated removal of cytosolically exposed presequence receptors (Chacinska et al., 2003; Komiya et al., 1998; Moczko et al., 1997). However, it should be mentioned that the exact OM translocation mechanism is presently elusive, as the deletion of the lone established trans-binding site at the TOM complex has no growth phenotype in yeast and only a minute retardation in import kinetics (Chacinska et al., 2003; Frazier et al., 2003; Moczko et al., 1997). This finding strongly suggests the existence of additional post Tom5 presequence-binding sites at the TOM complex.

Tom40, positioned functionally downstream of Tom5, has long been observed to interact with presequence-containing substrates. In 1989, Tom40 was the first identified mitochondrial translocation related protein (Vestweber et al., 1989). Vestweber and colleagues used an arrestable artificial chimeric mitochondrial substrate containing a photo-reactive crosslinker, and obtained strong photo-adduct formation to a protein of 42 $\mathrm{kDa}$. Shortly after, the deletion of the corresponding gene in yeast was shown to lead to the cytosolic accumulation of mitochondrial substrates, resulting in death (Baker et al., 1990). Today, it is known that Tom40 is the key channel forming subunit in the TOM complex (Neupert and Herrmann, 2007). Moreover, the TOM complex is the main entry gate into the mitochondrion, with all substrates destined past the OM passing through its translocation pore, largely explaining the early observation by Vestweber et al.

Ever since the discovery of Tom40's central role in mitochondrial protein translocation, it has been suggested to play an active role in mediating the translocation of presequence-containing substrates. Case in point, in 1997 Rapaport and others observed direct interaction of Tom40 with a presequence-containing substrate (Rapaport et al., 1997). Specifically, Tom40's interaction with presequence-containing substrates was shown to be independent of other cytosolically exposed, trypsin sensitive (Tom20, Tom22 
and Tom70), receptors of the TOM complex. Additionally, Tom40 was shown to make contact with the translocating substrate both at the cis and trans surfaces, utilizing a previously published differential salt wash technique (Mayer et al., 1995). Since the initial, aforementioned publications, postulating Tom40's direct interaction with mitochondrially targeted substrates, numerous additional studies have attempted to demonstrate its direct functional interaction in regards to presequence import (Gaikwad and Cumsky, 1994; Gordon et al., 2001; Juin et al., 1997; Kanamori et al., 1999; Rapaport et al., 1998a; 1998b; Stan et al., 2000). However, the characterization of a dedicated presequence-binding domain within the Tom40 channel has only been theorized (Ahting et al., 2001; Gabriel et al., 2003; Gessmann et al., 2011; Hill et al., 1998; Künkele et al., 1998; Mahendran et al., 2012; Sherman et al., 2006).

When the presequence emerges from the trans side of the TOM complex it can interact with the IMS domain of Tom22 as mentioned above. However, it is known that this interaction is not necessary for efficient import (Chacinska et al., 2003; Frazier et al., 2003; Moczko et al., 1997) and that a key essential presequence receptor of the translocase of the inner mitochondrial membrane (TIM23, presequence translocase) participates in the stabilization of late stage TOM intermediates (Chacinska et al., 2005). This early acting, primary presequence-interacting, IM bound presequence translocase receptor is Tim50 (Schulz et al., 2011). Tim50 contains a large IMS domain, which has been shown to possess two presequence-binding domains; one of which, at the C-terminus, was shown to be essential (Qian et al., 2011; Schulz et al., 2011). When Tim50 is bound by a presequence, it triggers key initial translocation steps within the presequence translocase (Lytovchenko et al., 2013; Meinecke et al., 2006; Mokranjac et al., 2009; Qian et al., 2011; Rahman et al., 2014).

The presequence translocase consists of three essential proteins, Tim23, Tim17 and Tim50 (Dudek et al., 2013). Tim23 is the major pore-forming subunit of the complex and its gating has been shown to be dependent on both the $\Delta \psi$ and presequences (Bauer et al., 1996; Truscott et al., 2001). Moreover, it contains a very large N-terminal IMS domain which was shown to interact with presequences and mediate import conferring initial translocase rearrangements (Komiya et al., 1998; Lytovchenko et al., 2013). Tim17 is involved in the stability of the Tim23 channel as well as acting as a sensor for various substrate types (Chacinska et al., 2005; Martinez-Caballero et al., 2007; Meier et al., 2005). However, an exhaustive description of Tim17's functions is still elusive. Tim50, in addition to the aforementioned role, has been shown to be functionally associated with the 
regulation of the TIM23 channel (Meinecke et al., 2006). In a study by Meinecke et al., Tim23 was reconstituted and its electrophysical characteristics were monitored, leading to the observation that the primarily open channel would spontaneously remain closed for extended periods of time after the addition of the IMS domain of Tim50 (Meinecke et al., 2006). This study highlighted Tim50's key role in maintaining the PMF over the IM.

Additional, nonessential components of the presequence translocase are Tim21 and the newly established Mgr2. Tim21 is known to couple the presequence translocase to the respiratory chain, specifically complexes III and IV (van der Laan et al., 2010). This association was shown to assist the insertion of presequence-containing substrates into the $\mathrm{IM}$, a process known not to require the import motor, but specifically dependent on the $\Delta \psi$ (Gambill et al., 1993; van der Laan et al., 2007; 2006). Moreover, Tim21 has been implicated in the early steps of IM based translocation; specifically it has been speculated to play a role in the removal of the presequence from the TOM trans-binding site (Tom22 $2^{\mathrm{IMS}}$ ) as it competitively binds (Albrecht et al., 2006; Chacinska et al., 2005; Mokranjac et al., 2005). Mgr2 was recently assigned as a presequence translocase component, as it was found to attach $\operatorname{Tim} 21$ to the presequence translocase as well as play a role in the transfer of presequence-containing proteins from the TOM complex to the presequence translocase (Gebert et al., 2012).

As exemplified by the complexity of interactions listed above, the precise path the presequence takes upon its exit from the TOM channel to the passage of the IM at the presequence translocase; namely the exact sequence of interactions with the presequence, is still not completely understood. However, the generally accepted route is as follows: (i) The emerging presequence from the Tom40 channel is captured by Tim50 through the possible assistance of Tom $22^{\mathrm{IMS}}$ and Tim21; (ii) Presequence-bound Tim50 associates with Tim23, causing the release of Tim21 from the presequence translocase and the hand-over of the presequence to the Tim23 channel, initiating channel opening in a $\Delta \psi$ dependent manner; (iii) The release of Tim21 causes the subsequent association of a key presequence associated motor (PAM) subunit, Pam17, triggering the subsequent association of the main translocation conferring PAM subunits, required for the full translocation of the substrate into the matrix. 


\subsubsection{The presequence translocase associated motor, PAM}

After the Tim23 channel has been opened, the presequence is initially pulled through the IM and into the matrix due to the electrophoretic force exerted on the presequence by the $\Delta \psi$ (Martin et al., 1991). Following the passage of the presequence into the matrix, the forward driving motion of the substrate is conferred by the PAM complex (Fig. 3, PAM constituents in green) (Chacinska et al., 2009; van der Laan et al., 2010; Wiedemann et al., 2004a). The PAM complex consists of the force conveying, ATPdependent subunit, mtHsp70 (Ssc1) (Kang et al., 1990; Liu et al., 2003; Ungermann et al., 1994; Voisine et al., 1999), and the cochaperones Pam18, Pam16, Pam17, Tim44 and Mge1 (van der Laan et al., 2010). Until 2003, the PAM complex was thought to contain only the mtHsp70, Tim44 and Mge1 (Miao et al., 1997; Schneider et al., 1994; 1996), in which Tim44 served to tether mtHsp70 to the presequence translocase and the nucleotide exchange factor Mge1 served to replenish the mtHsp70 import cycle. The discovery of several key PAM components quickly led to the first models on how the motor conveyed inward driving force. Two motor models arose; one of which, the Brownian ratchet model, advocated that the major precursor interacting subunit, mtHsp70, trapped the translocating polypeptide in the matrix and thereby prevented backsliding, solely relying on Brownian motion for inward movement (Ungermann et al., 1994). The second mechanistic PAM model, the power stroke model, hypothesized that the mtHsp70 actively pulled the substrates into the matrix and discarded the functionality of a forceless import model (Voisine et al., 1999). Although both models have now been discussed for nearly two decades, no study has yet to concretely discredit the other. Moreover, a compromise of both models, the Brownian ratchet model for loosely folded substrates and the power stroke model for tightly folded precursors, has been presented (van der Laan et al., 2010).

In the early 2000's, the discovery of three additional PAM components, Pam18, Pam16 and Pam17, strongly suggested that any simple mechanistic explanation of the PAM complex is likely not accurate. Pam 18 contains a single transmembrane span with a small IMS domain and a highly conserved matrix localized J-domain (D'Silva et al., 2003; Mokranjac et al., 2003; Truscott et al., 2003). The J-domain (named after prokaryotic protein DnaJ) interacts with mtHsp70 and stimulates the ATPase activity (Walsh et al., 2004). Pam16 was found in a stable complex with Pam18 and is required for the association of the heteroligomer to the presequence translocase (D'Silva et al., 2008; 2005; Frazier et al., 2004; Kozany et al., 2004; Mokranjac et al., 2007; 2006), however, interestingly Pam16 was found to inhibit the ATPase activity of mtHsp70 via its J-like 
domain and therefore likely acts in the fine-tuning of the import motor activity (Li et al., 2004). Furthermore, the crystal structure of the Pam $18 /$ Pam 16 complex clearly illustrates the tight association of the two proteins (Mokranjac et al., 2006), a complex strangely also shown to directly interact with the respiratory chain (Wiedemann et al., 2007). Pam17 is a membrane-integrated protein with two membrane-spanning domains, and was shown to be vital for the association of the Pam18/Pam16 complex to the presequence translocase (Popov-Celeketić et al., 2008a; van der Laan et al., 2005). Finally, Pam17, was recently shown to exhibit dynamic association with the presequence translocase, in direct response to presequence mediated priming events at the cis side of the TIM23 complex (Lytovchenko et al., 2013). These key translocase priming steps have long been observed (Dudek et al., 2013; van der Laan et al., 2010), and have led to the highly debated proposal of two functionally distinct TIM23 complexes (Chacinska et al., 2005).

\subsubsection{Presequence translocase dynamics, TIM $23^{\text {MOTOR }}$ and TIM23 ${ }^{\text {SORT }}$}

The presequence translocase is a unique translocase of the inner mitochondrial membrane as it is competent in both the matrix translocation of presequence-containing substrates as well as the membrane insertion of single transmembrane domain-containing substrates. The fulfillment of these distinct tasks is performed by two compositionally different presequence translocases, these being the TIM $23^{\text {MOTOR }}$ for the matrix translocation of precursors and TIM $23^{\text {SORT }}$ for the membrane insertion of transmembrane domain-containing precursors (Fig. 2) (Chacinska et al., 2005; 2010). TIM23 ${ }^{\text {SORT }}$ has been shown to migrate more slowly than other TIM23 complexes on BN-PAGE and consists of the core essential TIM23 subunits, Tim23, Tim17 and Tim50, as well as Mgr2 and Tim21. TIM23 ${ }^{\text {MOTOR }}$ contains all subunits of the TIM23 ${ }^{\text {SORT }}$ isoform, with the exception of Tim21, as well as the PAM (Dudek et al., 2013). The existence of multiple presequence translocase pools requires that the translocase possesses sensor-acting subunits, able to distinguish between a matrix and an IM integrated precursor. Moreover, the translocase must appropriately respond to the incoming sensors, gearing up for the proper translocation of the incoming substrate. The exact nature of the translocase associated sensor-acting subunits is still unknown; however, many studies have demonstrated the dynamics of the presequence translocase in the response to different substrate-based targeting signals (Chacinska et al., 2005; 2010; Lytovchenko et al., 2013; Popov-Celeketić et al., 2008a). Nearly all presequence translocase targeted substrates have a presequence, however, exceptions such as Sym1 have been characterized (Reinhold et al., 2012). Yet what 
distinguishes whether or not they are laterally released from TIM $23^{\text {SORT }}$ or translocated fully into the matrix by TIM $23^{\text {MOTOR }}$ is the presence or absence of a stop-transfer signal (Glick et al., 1992). The stop-transfer signal is located downstream of the presequence and consists of a hydrophobic stretch, which stalls the matrix translocation and then initiates the lateral release from TIM $23^{\text {SORT }}$ preceding through a presently elusive mechanism, known to involve Tim17 (Bömer et al., 1997; Chacinska et al., 2005). Interestingly, the composition of the stop-transfer signal has been shown to be enriched in tyrosine and phenylalanine residues, and that the addition of a proline within the stop-transfer sequence leads to complete matrix translocation of IM targeted proteins (Miller and Cumsky, 1993; Neupert and Herrmann, 2007).

The true existence of two separate presequence translocases was strongly supported by the publication of a motor-free in vitro reconstituted TIM23 import system (van der Laan et al., 2007). van der Laan and colleagues were able to demonstrate the minimal TIM23 import system for the import of membrane integrated substrates which they showed to be dependent upon a voltage-potential and a cardiolipin-rich membrane. Importantly, this system utilized isolated translocase from yeast mitochondria, free of PAM constituents (van der Laan et al., 2007). Moreover, Tim21 was shown to play a major role in differentiating the motor and sort isoforms, as it was found only in TIM23 ${ }^{\text {SORT }}$ (Chacinska et al., 2005; van der Laan et al., 2006; 2007). The multiple isoform TIM23 model is not unanimously supported by the field, as other publications have shown the association of PAM subunits with TIM23 ${ }^{\text {SORT }}$ and have advocated for a single translocase model (PopovCeleketić et al., 2008a; Tamura et al., 2009; 2006), however, only substoichiometric amounts were found to be associated.

Finally, cooperation between both presequence translocase isoforms has been observed (Bohnert et al., 2010). Bohnert et al. have uncovered the presequence translocase substrate Md11, a member of the $\mathrm{ABC}$ transporter family, which contains three pairs of two transmembrane spans. Interestingly, the first and third transmembrane spans are inserted into the IM via TIM $23^{\text {SORT }}$, and the second set is first completely translocated into the matrix via TIM $23^{\text {MOTOR }}$ where it is inserted into the IM through an Oxa1, a YidC homolog (van der Laan et al., 2003), dependent mechanism. The characterization of the Md11 import pathway presents a novel mechanistic description of the presequence translocase, namely its capacity to functionally interconvert between its two functional states during the import of a substrate. 


\subsubsection{Presequence import, the TOM-TIM23 supercomplex and TOM energetics}

The translocation across the mitochondrial OM via the TOM complex is believed to occur independent of direct ATP hydrolysis (Asai et al., 2004; Endo and Yamano, 2010). ATP hydrolysis is known to be required to preserve import compatibility through the retention of an unfolded state in many hydrophobic mitochondrial substrates, however, the direct requirement of ATP hydrolysis for OM passage was never directly shown (Endo and Yamano, 2010). This predicament would inevitably lead one to question the energetics of OM passage. An exhaustive description of TOM energetics is still elusive (Neupert and Herrmann, 2007), yet in the case of a particular subset of presequence-containing substrates an explanation has been presented (Dudek et al., 2013; van der Laan et al., 2010). The existence of two-membrane-spanning translocation supercomplex intermediates has long been observed (Pon et al., 1989; Rassow et al., 1989; Schleyer and Neupert, 1985; Schülke et al., 1997; Schwaiger et al., 1987), even predating the discovery of the first mitochondrial import-mediating protein in 1989 (Vestweber et al., 1989). Approximately a decade after translocation supercomplexes were observed, they were isolated and biochemically shown to consist of a tertiary complex of the precursor, TOM and TIM23 (Dekker et al., 1997; Sirrenberg et al., 1997). Today, we know that presequence-containing precursors of a particular size range are capable of translocase supercomplex formation when a stable fold is induced in the C-terminal portion of the substrate, preventing TOM passage (Dekker et al., 1997; Krayl et al., 2007). In fact, these translocase supercomplex intermediates are stable enough to survive digitonin solubilization and BN-PAGE and require a $\Delta \psi$, ATP and a C-terminal stable fold (Chacinska et al., 2003; 2005; 2010). The absolute dependence on an intact $\Delta \psi$ for the isolation of a translocation supercomplex is due to the functional coupling of both TOM and TIM23 passage (Chacinska et al., 2010), therefore presenting a possible explanation, in a defined precursor subset, to the energetics predicament of OM passage by TOM. This model couples the energy requirements of IM translocation to the "pulling" of the substrate through the OM. However, it is known that for short precursors, less than $\sim 80$ residues, a translocation supercomplex is not formed (Krayl et al., 2007), exemplifying our lack of understanding in OM passage and presenting a large area for further study. 


\subsubsection{Mitochondrial import of metabolite carrier proteins, the carrier pathway}

Mitochondrial substrates targeted to the mitochondrial IM have two possible routes, the presequence pathway (see section 1.3.1. for a detailed description) and the carrier pathway (Fig. 4). Their common destination, the mitochondrial IM, is one of the most, if not the most, protein-rich membranes known (Dudek et al., 2013). This fact is certainly due to the energy production demands of the mitochondrial respiratory chain (see section 1.2. for a description), fulfilling the majority of cellular energy needs in almost all human cell types. The term "carrier pathway" was coined due to the observation that multiple metabolites are transported across the mitochondrial IM through channels that share a common import and assembly pathway. The carrier pathway is utilized by proteins destined for the mitochondrial IM, lacking presequences and containing multiple transmembrane domains (Dudek et al., 2013). The structure of carrier proteins is best described by three pairs of membrane-spanning segments, each of which connected by a hydrophilic loop, with the even transmembrane domains forming salt bridges during each transport cycle (Ruprecht et al., 2014). This group of substrates is best exemplified by two well-characterized proteins, the ADP/ATP carrier (AAC) and the phosphate carrier (PiC); however, it is known that other proteins, such as Tim22, Tim17 and Tim23, also utilize this pathway. 


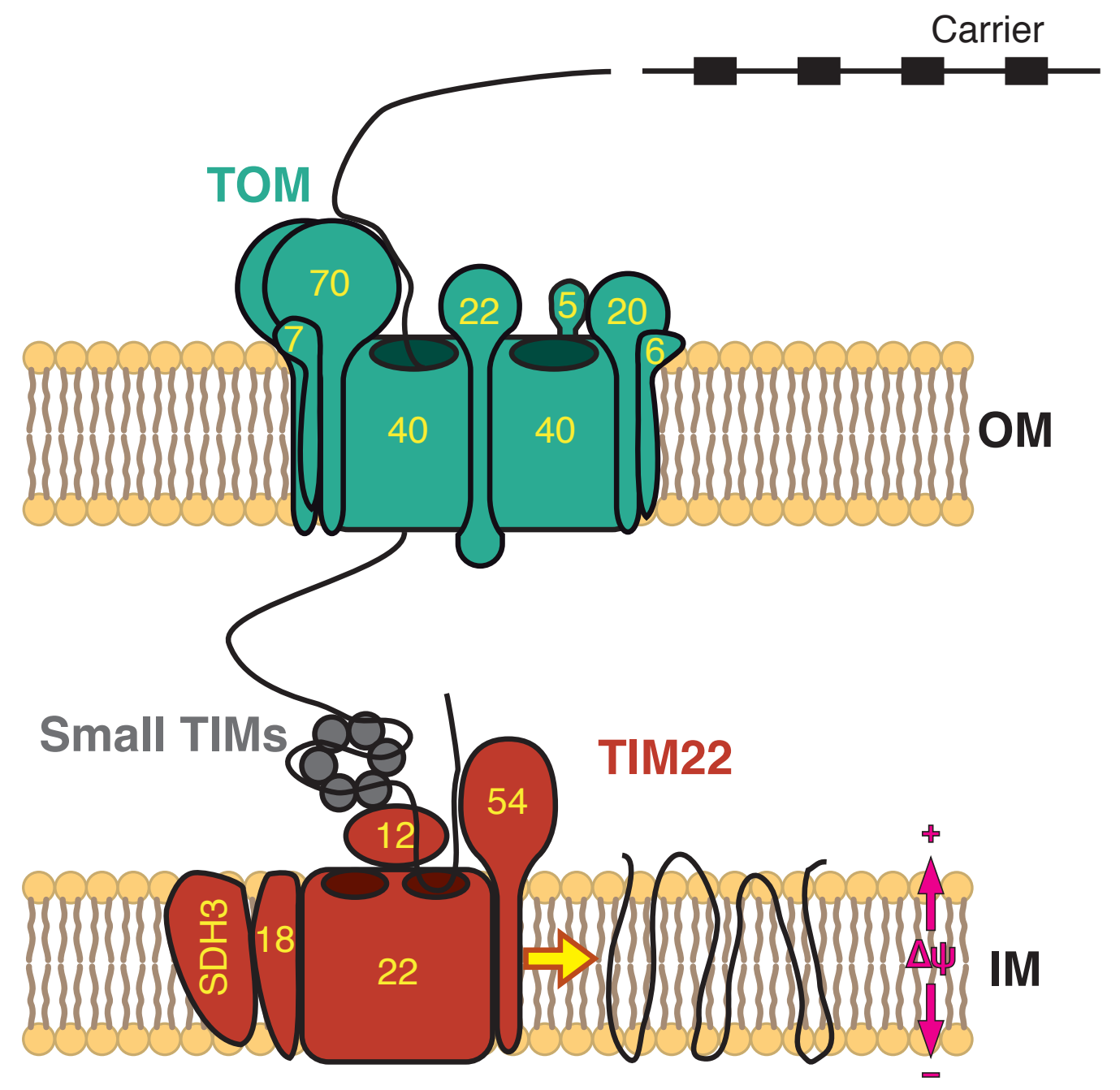

Figure 4. The mitochondrial carrier pathway. Substrates of the carrier pathway enter the mitochondrion through TOM in a Tom70 dependent manner. After entering the intermembrane space (IMS), small TIMs bind the hydrophobic substrate and shuttle it to the TIM22 complex via interaction with Tim12. The substrate is positioned via the receptor Tim54 and the transmembrane domains are inserted loop-wise into the inner membrane (IM) in a $\Delta \psi$ dependent manner. OM, outer membrane.

The import of carrier proteins into the mitochondrion has been characterized to exhibit five distinct and isolatable import stages (Pfanner et al., 1987; Ryan et al., 1999). During stage one, the highly hydrophobic nascent substrate is kept in a soluble state via the concerted activity of Hsp90 and Hsp70 in mammals (in yeast, Hsp70 alone is utilized) (Young et al., 2003; Zara et al., 2009; Zimmermann and Neupert, 1980). Following chaperone binding, stage two entails the substrate-chaperone complex being targeted to the outer mitochondrial membrane via chaperone interactions with the TOM complex receptor, a Tom70 dimer, utilizing its tricopeptide repeat (TPR) domains (Young et al., 2003). A study by Young and colleagues (2003) demonstrated that a key, highly conserved (from 
yeast [R171] to human [R192]) Tom70 residue mediates its interaction with substrateloaded chaperones. In a peptide scanning approach, Tom70 was found to exhibit affinity to several peptides of internal-targeting-signal containing carrier pathway substrates (Brix et al., 1997; 1999; 2000). Moreover, other studies have found strong evidence for the interaction of a single carrier substrate with three copies of a Tom70 dimer as the substrate is prepared for OM passage (Wiedemann et al., 2001).

Stage three of the carrier pathway is observed in in vitro import reactions under conditions in which the $\Delta \psi$ has been depleted and when ATP is exogenously added (Ryan et al., 1999). Stage three has been divided into two steps, the first of which involves the binding of the IMS chaperone Tim9-Tim10 heterohexamer complex when loops of the substrate transverse the OM through the TOM channel (Curran et al., 2002; Endres et al., 1999; Koehler et al., 1998; Luciano et al., 2001; Sirrenberg et al., 1998). This chaperonelike complex association with the translocating substrate allows for the release of the substrate from the trans side of the TOM complex (Truscott et al., 2002). Moreover, a homologous IMS chaperone complex, Tim8-Tim13, has been implicated in the translocation of $\operatorname{Tim} 23$ and therefore likely functions similarly to the Tim9-Tim10 complex during the import of non-carrier proteins which utilize the carrier pathway for IM import and assembly (Beverly et al., 2008; Davis et al., 2007; Hoppins and Nargang, 2004). The second step of stage three involves the association of Tim 12 , allowing for the small TIM bound translocation intermediate to interact with the translocase of the inner mitochondrial membrane (TIM22, carrier translocase) (Gebert et al., 2008; Sirrenberg et al., 1998). It was later noticed that the accumulation of the protease resistant stage three intermediate, seen in conditions with a depleted $\Delta \psi$ and exogenous ATP after BN-PAGE, represented the small TIM bound substrate tethered to the carrier translocase (Rehling et al., 2003).

The TIM22 translocase was shown via electron microscopy (EM) to contain two pores, each capable of passing two $\alpha$-helices simultaneously, formed by its pore-forming subunit Tim22 (Rehling et al., 2003). The carrier translocase is comprised of the central channel-forming subunit Tim22 and the assembly and receptor subunits Tim54, Tim18 and Sdh3 (Dudek et al., 2013). Tim54 consists of an extended IMS domain which has been shown to be the docking point for small TIM bound carrier translocase substrates (Hwang et al., 2007; Wagner et al., 2008). Tim18, an Sdh4 homolog of complex II (succinate dehydrogenase) of the respiratory chain, was observed to function as a Tim54 assembly 
factor, assisting its integration into the TIM22 complex (Wagner et al., 2008). Sdh3, a subunit of the respiratory chain succinate dehydrogenase, was recently added to the list of TIM22 components as it forms a subcomplex with Tim18 during the assembly of the carrier translocase (Gebert et al., 2011). During stage four of carrier import, the twin-pore channel is opened in a $\Delta \psi$ and signal-dependent manner (Kovermann et al., 2002), at which point loops of the substrate are inserted into the IM, likely due to the electrophoretic force imparted on the positive charges in the loop regions by the $\Delta \psi$. Stage five entails the lateral release of the substrate into the IM by a presently elusive mechanism and, in the case of metabolite carriers, subsequent homodimer formation (Dudek et al., 2013).

\subsubsection{Mitochondrial import and assembly of outer membrane $\beta$-barrel proteins}

Gram-negative bacteria, a distant ancestor of modern day endosymbiotic organelles, are surrounded by two encompassing membrane envelopes. The bacterial outer envelope is residence to over 70 different $\beta$-barrel-type proteins (Wimley, 2003). A hallmark, retained uniquely within eukaryotic organelles of endosymbiotic origin, are $\beta$ barrel OM proteins (Dolezal et al., 2006). Thus far, mitochondrial researchers have uncovered four $\beta$-barrel OM proteins (Tom40, Sam50, Mdm10 and porin [voltagedependent anion-selective channel, known as VDAC1 in humans]) which contain a distinct motif, known as the $\beta$-signal, and utilize a partially evolutionary conserved import and assembly pathway (Fig. 5) (Kutik et al., 2008). The established C-terminal OM import and assembly targeting motif (Polar, x, Glycine, x, x, Large hydrophobic, x, Large hydrophobic $[\mathrm{x}=$ any residue $]$ ) of these proteins commences at the main mitochondrial entry gate, TOM (Kutik et al., 2008). After being translated on cytosolic ribosomes, the $\beta$-barrel-type protein is inserted into the IMS via the TOM complex, utilizing all three major TOM receptors (Tom20, Tom22 and Tom70). Moreover, Tom40 (the pore-forming subunit of the TOM complex), has been shown to exhibit chaperone-like functions when translocating hydrophobic substrates (Esaki et al., 2003), likely preventing the aggregation of $\beta$-barrel membrane proteins during outer membrane passage. 


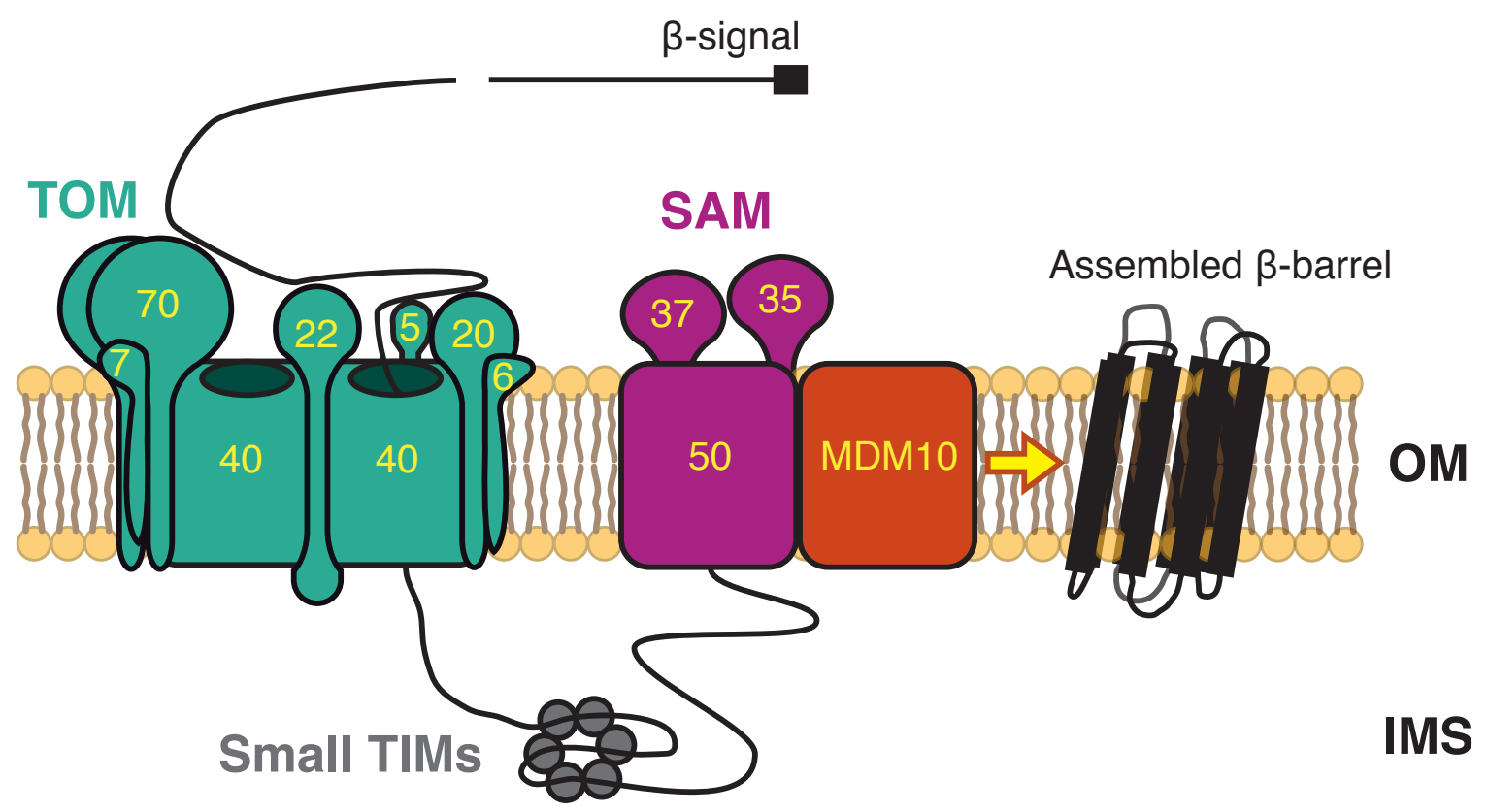

Figure 5. The mitochondrial sorting and assembly (SAM) pathway. $\beta$-signal-containing substrates $(\beta$-barrel proteins of the outer membrane $[\mathrm{OM}])$ are imported first via TOM through the actions of Tom70, Tom22 and Tom20. Upon entering the intermembrane space (IMS) they interact with the heterohexamer chaperone complex, known as the small TIMs, and are directly handed off to the sorting and assembly machinery (SAM). Through the actions of the SAM complex, the $\beta$-barrel substrate is inserted into the OM and assembled into its target complex.

When the $\beta$-signal-containing substrate enters the IMS, local chaperones (Tim9Tim10 heterohexamers) prevent aggregation in route to the sorting and assembly (SAM) of the OM (Webb et al., 2006; Wiedemann et al., 2004b). The SAM complex is conserved among eukaryotes and comprised of two essential core components Sam50 and Sam35, as well as Sam37 and Mdm10 (Chacinska et al., 2009; Dudek et al., 2013; Meisinger et al., 2004; Waizenegger et al., 2004). Sam50 is a homolog of the bacterial Omp85 (BamA) and a $\beta$-barrel protein with channel conducting activity corresponding to a large pore diameter of 40 - $50 \AA$ (Kutik et al., 2008). Sam50's N-terminus contains a single polypeptide transport associated (POTRA) domain, as opposed to Omp85, which has five. In bacteria, the most C-terminal POTRA domain of Omp85 (retained in the mitochondrial homolog Sam50) was shown to be essential, as opposed to the N-terminal four which elicited only mild growth effects upon their deletion (Bos et al., 2007). In mitochondria, the POTRA domain of Sam50 was shown to bind specifically to $\beta$-barrel precursors, hinting on its receptor function (Habib et al., 2007). However, shortly after this finding, Kutik et al. demonstrated that this domain is not only non-essential, but also not required for SAM 
complex activity, uncovering Sam35 as the IMS facing $\beta$-signal receptor (Kutik et al., 2008). In the study by Kutik and others, Sam 35 was shown to act as a receptor and exhibit $\beta$-signal affinity, specifically when expressed and purified from E. coli. Sam37 (Mas37), initially identified as a TOM constituent (Wiedemann et al., 2003) (likely due to the recent discovery of the association of TOM and SAM [Qiu et al., 2013]), is now known to be the substituent of the SAM complex, involved in the release of substrates for the SAM complex (Chan and Lithgow, 2008). Mdm10, associated with both the SAM and the endoplasmic reticulum-mitochondria encounter structure (ERMES), is known to promote late stages of Tom40 assembly (Meisinger et al., 2004).

Moreover, recent investigation into the functionally elusive POTRA domain of Sam50 has brought to light its underlying evolutionary fitness-conferring role. Stroud et al. utilized a wheat germ lysate system to produce $\beta$-barrel substrates for use in subsequent import assays utilizing mitochondria which lacked the POTRA domain of Sam50 (Stroud et al., 2011). In their assay, they were able to stress the SAM complex with an abundance of incoming substrate, which allowed for the visualization of precursor accumulation within the SAM complex. This data then allowed for the assignment of a substrate release role of the highly evolutionary conserved POTRA domain of Sam50.

It should be noted that other $\beta$-barrel-type proteins have been characterized to reside in the outer mitochondrial membrane, such as Mmm2 (Mdm34) (Youngman et al., 2004). Mmm2, an established ERMES component (Kornmann et al., 2009; Wideman et al., 2013), has a $\beta$-signal distal to the C-terminus (Imai et al., 2008), suggesting a divergent or slightly modified import and/or assembly mechanism exists for mitochondrial $\beta$-barrel OM proteins.

\subsubsection{Mitochondrial intermembrane space import and assembly pathway}

The mitochondrial intermembrane space import and assembly (MIA) pathway is a key import pathway for substrates destined for the IMS that contain a defined multiple cysteine motif, $\mathrm{Cx}_{\mathrm{n}} \mathrm{C}$ (Fig. 6) (Milenkovic et al., 2009; Sideris et al., 2009). The core MIA machinery consists of the essential subunits Mia40 and Erv1 which are involved in the formation of transient disulfide bonds with incoming substrates and subsequently function in a disulfide relay system which is responsible for the both the assembly and retention of many IMS substrates (Allen et al., 2005; Chacinska et al., 2004; Grumbt et al., 2007; Mesecke et al., 2005; Milenkovic et al., 2007; Naoé et al., 2004). Mia40 engages with its 
substrates at the trans side of the TOM channel, positioned by Fcj1 of the mitochondrial inner membrane organizing system (MINOS) (Malsburg et al., 2011), by forming intermolecular disulfide bonds in its redox-active cysteine - proline - cysteine motif hydrophobic groove (Banci et al., 2011; 2009; Kawano et al., 2009; Terziyska et al., 2009). Immediately following the intermolecular disulfide bond between Mia40 and its substrate, Mia40 catalyses intramolecular disulfide bridge formation within the substrate (Chacinska et al., 2004; Müller et al., 2008). This mode of disulfide bridge formation is now known to trigger the retention of various dual-localized proteins such as superoxide dismutase 1 and its copper chaperone Ccs1 (Gross et al., 2011; Kawamata and Manfredi, 2010; Klöppel et al., 2011; Reddehase et al., 2009), thereby instilling a key regulatory role within MIA pathway.

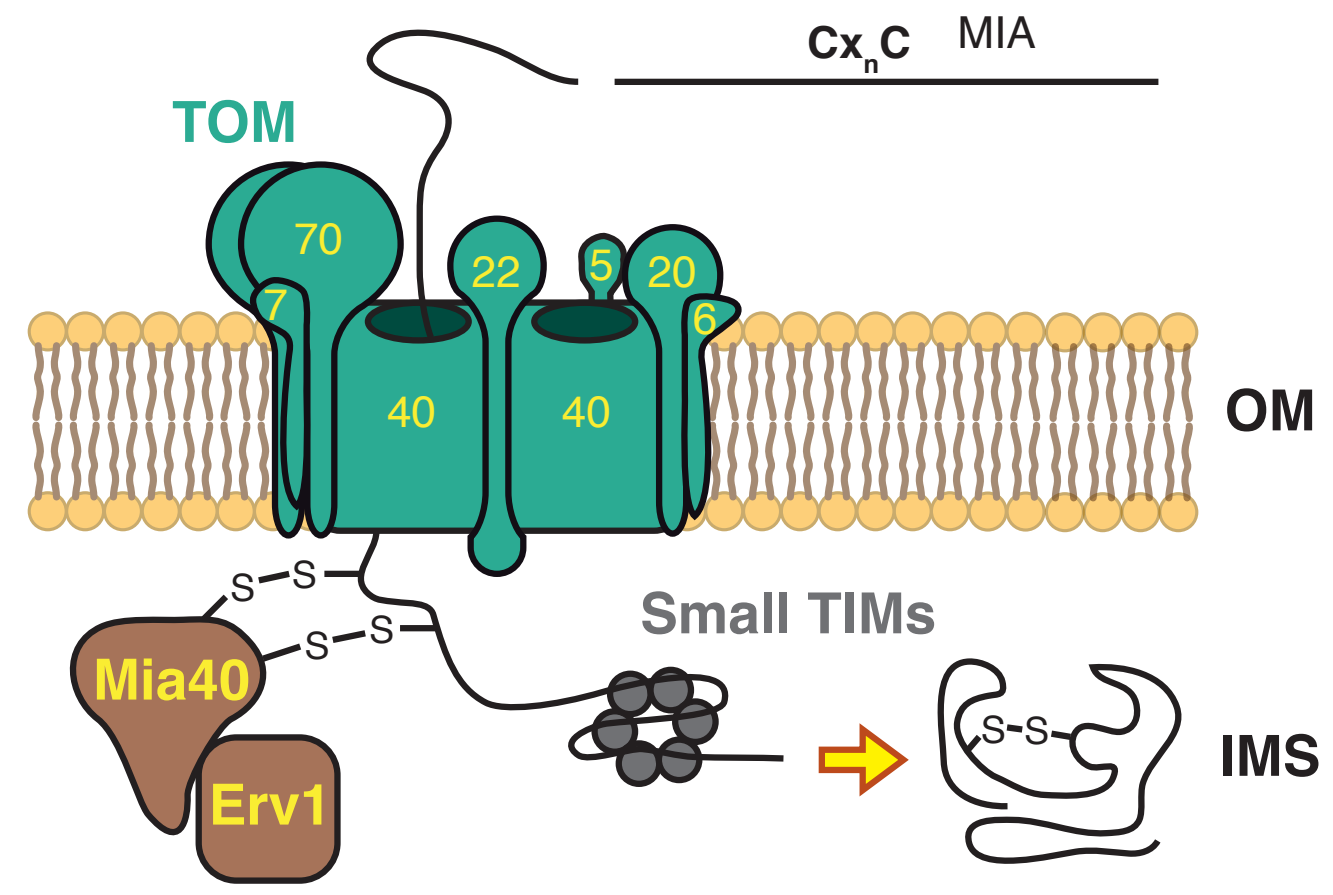

Figure 6. The mitochondrial intermembrane space import and assembly (MIA) pathway. $\mathrm{Cx}_{\mathrm{n}} \mathrm{C}$ signal-containing substrates cross the outer membrane $(\mathrm{OM})$ at TOM and are oxidized in the IMS via Mia40 and allowed to form intramolecular disulfide bridges and proceed to the small TIMs for final folding. Erv1 associates with Mia40 in complex with a substrate and allows for the reoxidation of Mia40.

Following the catalysis of the substrate intramolecular disulfide bridge by Mia40, its reoxidation is carried out by Erv1 (Bien et al., 2010; Dabir et al., 2007). Erv1 has been previously shown to form a ternary complex with Mia40 and its substrate (Stojanovski et 
al., 2008), streamlining the Mia40 import cycle. The active Erv1 homodimer shuttles electrons from Mia40 to molecular oxygen via cytochrome $c$ and cytochrome $c$ oxidase of the respiratory chain (Banci et al., 2011; Bihlmaier et al., 2007; Mesecke et al., 2005). Thus, the coupling of the MIA disulfide relay system to the respiratory chain ensures the maintenance of oxidized Mia40, thereby allowing for continuous import rounds and the suppression of toxic reactive oxygen species (ROS). The now intramolecular disulfide bridge containing substrate then can interact with the chaperone acting small TIMs of the IMS, allowing for proper folding to take place and subsequent release of the soluble protein (Chacinska et al., 2009).

\subsubsection{Mitochondrial import of $\alpha$-helical outer membrane proteins}

The sorting of $\alpha$-helical OM proteins containing either an $\mathrm{N}$-terminal anchor sequence (Becker et al., 2008; Hulett et al., 2008) or, in some cases, multiple transmambrane domains (Becker et al., 2011; Papic et al., 2011) is carried out by the Mim1 complex (Fig. 7). Mim1, originally identified to be involved in assembly of $\beta$-barrel OM proteins (Ishikawa et al., 2004), has been recently shown to mediate the OM integration of $\alpha$-helical, single and multiple transmembrane-containing proteins (Becker et al., 2011; 2008; 2010; Hulett et al., 2008; Lueder and Lithgow, 2009; Papic et al., 2011; PopovCeleketić et al., 2008b; Thornton et al., 2010). Moreover, Tom70 has been implicated in the Mim1 import pathway, serving as a receptor for incoming substrates and being a Mim1 substrate itself (Becker et al., 2011; 2008). Therefore, the Mim1 complex has been firmly established to play a crucial role in the OM integration of many $\alpha$-helical proteins. In particular the biogenesis of the translocase of the outer mitochondrial membrane (TOM) complex as Tom5, Tom6, Tom7, Tom20 and Tom70 require Mim1 for efficient OM integration (Becker et al., 2008; Hulett et al., 2008). Following the identification of Miml's intimate role in the OM integration of the majority of TOM proteins, the original finding of its role in $\beta$-barrel sorting was shown to be the result of an indirect effect (Becker et al., 2010), as Tom40 (a $\beta$-barrel protein consisting of the pore-forming TOM subunit) is known to display assembly defects in the absence of the small TOM subunits (Tom5, Tom6 and Tom7) (Alconada et al., 1995; Dekker et al., 1998; Dembowski et al., 2001; Dietmeier et al., 1997; Hönlinger et al., 1996; Kato and Mihara, 2008; Schmitt et al., 2005; Sherman et al., 2005). 


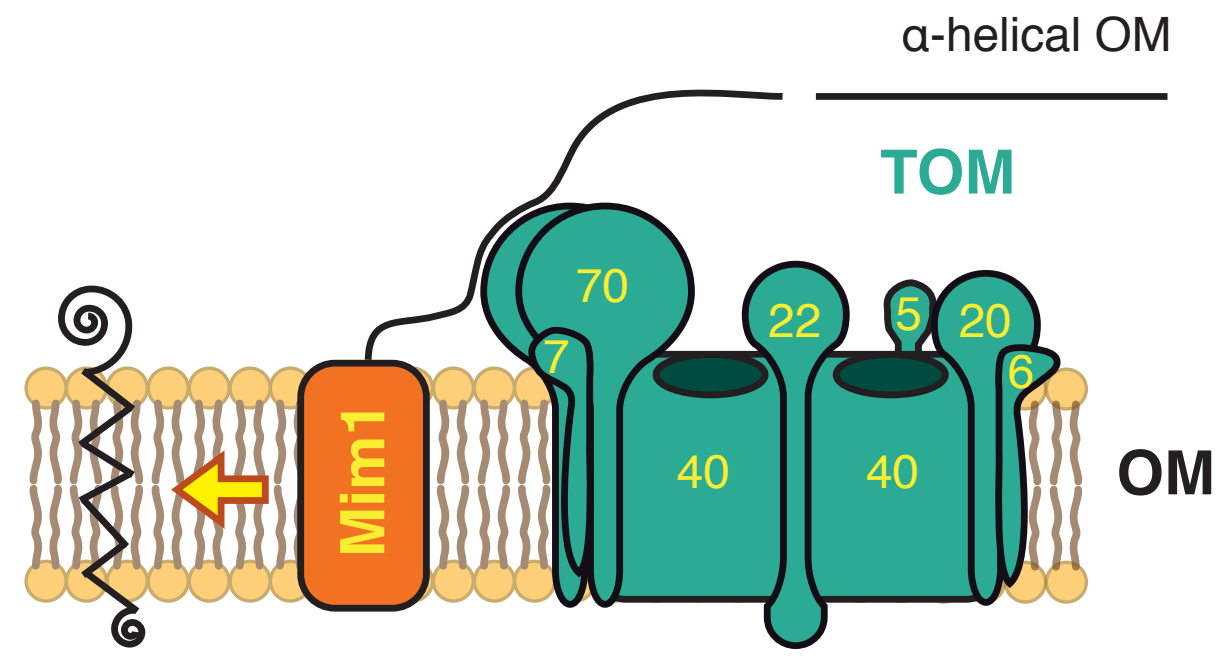

Figure 7. The import of mitochondrial $\alpha$-helical outer membrane (OM) proteins. Single and multiple transmembrane domain containing $\alpha$-helical OM proteins, containing an $\mathrm{N}$ - or $\mathrm{C}$-terminal anchor, are integrated into the OM via the Mim1 complex in a Tom70 dependent manner.

Finally, the last variant of $\alpha$-helical OM proteins, single transmembrane domain containing C-terminally anchored proteins, appear to be integrated into the mitochondrial $\mathrm{OM}$ without a proteinaceous integration machinery (with the exception of Tom22) (Kemper et al., 2008; Setoguchi et al., 2006). Kemper et al., utilizing the model single transmembrane containing C-terminally anchored $\alpha$-helical OM protein Fis1, observed that a large number of characterized $\mathrm{OM}$ translocation mutants had no impact on Fis1 integration and that Fis 1 was able to efficiently integrate in lipid vesicles (Kemper et al., 2008). Moreover, they observed vesicular ergosterol content to negatively impact membrane integration of Fis1, hinting on a non-proteinaceous lipid regulated import system. In conclusion, there appear to be multiple avenues for the insertion of $\alpha$-helical OM proteins, segregated by a combination of the position of the anchor sequence and the number of membrane spanning domains. 


\subsection{Research objectives}

This work aims to advance our understanding of mitochondrial OM passage of presequence-containing substrates. In brief, our current knowledge of presequence translocation at the TOM complex is lacking a step-by-step delineation of presequence interactions at the $\mathrm{OM}$, leading to presequence engagement with IM bound presequence receptors. Presently, a wealth of information exists in regard to the presequences' interaction with cytosolically-oriented TOM receptors. However, in stark contrast, very little is known about the mechanistic steps made by the presequence-containing precursor after it has been handed over to the pore-forming subunit of the TOM complex, Tom 40 . Moreover, the lone trans-acting presequence receptor, the IMS domain of Tom22, was shown to exhibit a remarkably insignificant impact on presequence mediated import (Frazier et al., 2003; Moczko et al., 1997), yet no other TOM presequence-interacting site has been characterized downstream of the cytosolically exposed receptors. This gap in OM translocation understanding is strongly preventing the establishment of a general functional TOM model. This predicament is highlighted by the utter lack of understanding as to where substrates pass the OM, specifically if the Tom40 pore is directly used or if the formation of higher ordered TOM ${ }^{\mathrm{CORE}}$ (consisting of Tom40 and Tom22) structures allow for channel formation within the OM, an idea previously suggested, which allows for the lateral release of TOM substrates (Harner et al., 2011). A more complete understanding of the sequential steps taken by the presequence-containing substrate, particularly the interactions within the Tom40 pore, would allow for the more accurate interpretation of future thought-provoking OM passage observations. Here, an in-depth structural and functional examination of Tom 40 presequence interactions is undertaken with the aims of better understanding presequence mediated TOM passage.

The second objective of the present work examines the steps in presequence transport immediately following TOM mediated OM passage, described above. After the presequence-containing substrate exits the TOM channel, it must be recognized and brought to the presequence translocase. At the presequence translocase, IMS exposed domains of its constituents must sense the incoming substrate and then trigger dynamic translocase rearrangements in response to the intended destination of the substrate, lateral release into the IM or translocation into the matrix by way of the associated PAM complex. This process of translocase priming due to an incoming substrate is presently not fully understood, however, numerous publications have demonstrated the dynamic nature of TIM23 subunits, specifically Tim21 and Tim50 (Chacinska et al., 2005; Mokranjac et 
al., 2005; Schulz et al., 2011). The present investigation seeks to advance upon the previously published knowledge of TIM23 dynamics and attempts to link presequence presentation in the IMS with the sequence of events that takes place at the presequence translocase during the early translocation priming stages. 


\section{MATERIALS AND METHODS}

\subsection{Materials}

\subsubsection{Chemicals}

All commonly used chemicals were purchased from the following companies; AppliChem (Darmstadt, Germany), Roth (Karlsruhe, Germany), Sigma-Aldrich (Taufkirchen, Germany), Merck (Darmstadt, Germany) and SERVA (Heidelberg, Germany). All chemicals obtained from the aforementioned companies were of analytical grade. Chemicals considered non-standard are listed in Table 1 along with the corresponding producer.

Table 1. List of non-standard chemicals and their respective producers used in this study.

\begin{tabular}{l|l} 
Chemical & Producer \\
\hline Antimycin A & Sigma Aldrich \\
ATP & Roche \\
BSA (fatty acid free) & Sigma Aldrich \\
CNBr-Activated Sepharose 4B & GE-Healthcare \\
Coomassie brilliant blue G250/R250 & Serva \\
Complete EDTA free protease inhibitor & Roche \\
Creatine phosphate & Roche \\
Digitonin & Calbiochem \\
Dimethyl pimelimidate (DMP) & Sigma Aldrich \\
Dropout mixes; CSM-Trp, CSM-URA & MP Biomedicals \\
Enhanced chemiluminescence detection reagent & GE-Healthcare, Millipore, \\
5-Fluoroorotic acid (5-FOA) & Pierce/Thermo Scientific \\
GeneRuler DNA Ladder Mix & Fermentas \\
Herring sperm DNA & Fermentas \\
High molecular weight calibration kit & Promega \\
IgG protein standard (bovine) & GE-Healthcare \\
& Biorad \\
\end{tabular}


Leupeptine

$\mathrm{NADH}$

Ni-NTA agarose

Oligomycin

Oligonucleotides

Pefablock

PEG-4000

Peptone, yeast extract and yeast nitrogen base w/o AAs

Phenylmethylsulfonyl fluoride (PMSF)

ProteinA-sepharose

ProteinG-sepharose

Roti-Quant

SDS marker

$\left[{ }^{35} \mathrm{~S}\right]$ Methionine

Streptavidin horseradish peroxidase (SA-HRP)

Triton X-100

Valinomycin
Sigma Aldrich

Roche

Qiagen

Sigma Aldrich

Metabion

Roche

Applichem

$\mathrm{BD}$

Roth

GE-Healthcare

GE-Healthcare

Roth

Serva

Hartmann Analytics

Dianova

Serva

Calbiochem

\subsubsection{Consumables}

All consumables used in this study are listed in Table 2, along with their respective producers.

Table 2. List of consumables used in this study, together with producers.

\begin{tabular}{l|l} 
Consumable & Producer \\
\hline CELL STAR $®$ centrifuge tubes $15 \mathrm{ml}$ and $50 \mathrm{ml}$ & Greiner \\
Centrifugal filters & Millipore \\
Medical x-ray films & Foma \\
Micro tube $1.5 \mathrm{ml}$ and $2.0 \mathrm{ml}$ & Sarstadt \\
Minisart ${ }^{\circledR}$ syringe filters & Sartorius \\
Mobicol spin columns & MoBiTec \\
PD G10 desalting columns & GE-Healthcare \\
Pipette tips & Sarstadt
\end{tabular}


Polyvinylidene fluoride (PVDF) Membrane

SnakeSkin dialysis tubing 7k MWCO
Millipore

Thermo Scientific

\subsubsection{Kits}

All Kits used in this work are listed in Table 3 with their suppler. All listed kits were used according to the manufactures' instructions.

Table 3. List of kits and suppliers used in this work.

\begin{tabular}{l|l} 
Kit & Suppler \\
\hline KOD Hot Start DNA polymerase & Novagen \\
mMESSAGE mMACHINE® SP6 Kit & Ambion \\
QuikChange® Lightning site-directed mutagenesis & Stratagene/Agilent \\
TNT® Quick coupled Transcription/Translation system & Promega \\
Wizard $®$ Plus SV Minipreps DNA Purification system & Promega \\
Wizard® SV Gel and PCR Clean-up system & Promega
\end{tabular}

\subsubsection{Enzymes}

All enzymes used in this work are listed in Table 4, in addition to the corresponding manufacturer.

Table 4. List of enzymes and manufactures used in this work.

\begin{tabular}{l|l} 
Enzyme & Manufacturer \\
\hline & \\
Creatine Kinase & Roche \\
DNase I & Roche \\
Trypsin & Sigma Aldrich \\
Proteinase K & Roche \\
Zymolyase 20T & Seikagaku Biobusiness
\end{tabular}




\subsubsection{Peptides}

The peptides, corresponding sequences and producers are listed in Table 5. Purchased peptides were synthesized as an $\mathrm{N}$-terminal amine and a $\mathrm{C}$-terminal amide. When the peptide was self-synthesized a corresponding reference is listed.

Table 5. List of peptides, sequences and producers/literature reference.

\begin{tabular}{l|l|l} 
Peptide & Sequence & Producer/Reference \\
\hline HA & YPYDVPDYA & \\
Synb2 & MLSRQQSQRQSRQQSQRQSRYLL & JPT Peptide Technologies \\
pCox4 & MLSLRQSIRFFKPATRTLSSSRYLL & (Allison and Schatz, 1986) \\
pL ${ }_{19} \mathrm{~B}$ & $\begin{array}{l}\text { MLRAALSTARRGPRLSRLBpaSAAARKbioHH } \\
\text { HHHH }\end{array}$ & $\begin{array}{l}\text { (Allison and Schatz, 1986) } \\
\text { (Schulz et al., 2011) }\end{array}$ \\
pS $_{16} \mathrm{~B}$ & $\begin{array}{l}\text { MLRAALSTARRGPRLBpaRLLSAAARKbioHH } \\
\text { HHHH }\end{array}$ & (Schulz et al., 2011) \\
& HHHidechnologies
\end{tabular}

\subsubsection{Antibodies}

All primary antibodies directed against yeast and mouse proteins were produced though antigen injection into rabbits and are listed in Table 6. Antigens were either in the form of a purified recombinant protein or a synthesized peptide. HA antibody was obtained from 12CA5 hybridoma supernatant. Secondary antibodies for use in Western blot detection, goat anti rabbit HRP and goat anti rabbit IR680 were purchased from Dianova (Hamburg, Germany) and LI-COR (Bad Homburg, Germany), respectively.

Table 6. List of primary antibodies and corresponding epitopes used in this study.

\begin{tabular}{l|l|l} 
Directed against & Epitope discription & Reference \\
\hline & & \\
Aco1 & Peptide: sequence not available & AG Rehling \#945 \\
Atp5 & Peptide: CDLSISTKIQKLNKVLEDSI & AG Rehling \#1546 \\
Atp20 & Peptide: CSVGEIIGRRKLVGYKHH & AG Rehling \#1517
\end{tabular}




\begin{tabular}{l|l|l} 
HA & YPYDVPDYA & Mouse monoclonal 12CA5 \\
Mcr1 & Whole protein & AG Pfanner \#613 \\
mDHFR & Peptide: EEKGIKYKFEVYEKKD & AG Rehling \#1846 \\
Mdm38 & Peptide: IPADQAAKTFVIKKD & AG Rehling \#342 \\
Pam17 & Information not available & AG Rehling \#279 \\
Por1 & Information not available & AG Rehling \#B94-E \\
Rip1 & Peptide: LEIPAYEFDGDKVIVG & AG Rehling \#543 \\
Ssc1 & Whole protein & AG Rehling \#119 \\
Tim17 & Peptide: PLPEAPSSQPLQA & AG Rehling \#302 \\
Tim21 & IMS domain & AG Rehling \#3111 \\
Tim23 & IMS domain & AG Rehling \#3845 \\
Tim44 & Peptide: EGWKILEFVRGGSRQFT & AG Rehling \#127 \\
Tim50 & IMS domain & AG Rehling \#3314 \\
Tom5 & Peptide: MFGLPQQEVSEEEKRAC & AG Rehling \#3635 \\
Tom20 & Cytosolic domain & AG Rehling \#3534 \\
Tom22 & Cytosolic domain & AG Rehling \#3533 \\
Tom40 & Whole protein & AG Rehling \#4901 \\
Tom70 & Cytosolic domain & AG Rehling \#3530 \\
& &
\end{tabular}

\subsubsection{Plasmids}

The plasmids used in this study are listed in Table 7, along with the originating vector, insert and relevant reference.

Table 7. List of plasmids used in this study.

\begin{tabular}{l|l|l|l} 
Plasmid & Backbone & Insert & Reference \\
\hline A01 & pGEM4X & N. crassa AAC & (Steger et al., 1990) \\
B07 & pUHE 73-1 & $\mathrm{b}_{2}(167)_{\Delta}-$ DHFR & (Koll et al., 1992) \\
pAF1 & OXA1 & (Frazier et al., 2003) \\
pYM10 & pYM10 & HIS3MX6 & (Janke et al., 2004) \\
Tim9 & pGEM4Z & TIM9 & (Wrobel et al., 2013) \\
Tom40-Ura & pFL39 (Ura) & TOM40 & (Kutik et al., 2008) \\
Tom40-Trp & pFL39 (Trp) & TOM40 & (Kutik et al., 2008)
\end{tabular}




\begin{tabular}{|c|c|c|c|}
\hline tom $40^{\text {Emut }}$ & pFL39 (Trp) & tom $40^{\text {Emut }}$ & This study \\
\hline tom $40^{V m u t}$ & pFL39 (Trp) & tom $40^{\text {Vmut }}$ & This study \\
\hline- & pFL39 (Trp) & - & $\begin{array}{l}\text { (Bonneaud et al., } \\
\text { 1991) }\end{array}$ \\
\hline $\operatorname{Tim} 21^{\mathrm{IMS}}$ & pProExHTa & $\operatorname{tim} 21^{I M S}$ & $\begin{array}{l}\text { (Albrecht et al., } \\
\text { 2006) }\end{array}$ \\
\hline $\operatorname{Tim} 23^{\mathrm{IMS}}$ & pET10C & $\operatorname{tim} 23^{I M S}$ & $\begin{array}{l}\text { (Truscott et al., } \\
2001)\end{array}$ \\
\hline $\operatorname{Tim} 23^{\text {YL70AA }}$ & $\mathrm{pET} 10 \mathrm{C}$ & $\operatorname{tim} 23^{I M S-Y L 70 A A}$ & $\begin{array}{l}\text { (Lytovchenko et al., } \\
\text { 2013) }\end{array}$ \\
\hline $\operatorname{Tim} 50^{\mathrm{IMS}}$ & pProExHTc & $\operatorname{tim} 50^{I M S}$ & (Schulz et al., 2011) \\
\hline
\end{tabular}

\subsubsection{Yeast Strains}

Yeast strains utilize in this work, along with the corresponding genotypes and relevant references are listed in Table 8 .

Table 8. List of yeast strains used in this study.

\begin{tabular}{|c|c|c|}
\hline Strain & Genotype & Reference \\
\hline YPH499 & $\begin{array}{l}\text { MATa, ade2-101, his3- } \Delta 200, \text { leu2- } \Delta 1, \text { ura3-52, } \\
\text { trp1- } \Delta 63, \text { lys } 2-801\end{array}$ & $\begin{array}{l}\text { (Sikorski and Hieter, } \\
\text { 1989) }\end{array}$ \\
\hline $\operatorname{tim} 21 \Delta$ & $\begin{array}{l}\text { MATa, ade2-101, his3- } \Delta 200, \text { leu2- } \Delta 1, \text { ura3-52, } \\
\operatorname{trp} 1-\triangle 63, \text { lys2-801; tim } 21:: H I S 3 M X 6\end{array}$ & $\begin{array}{l}\text { (Chacinska et al., } \\
2005)\end{array}$ \\
\hline $\operatorname{Tim} 21^{\text {ProtA }}$ & $\begin{array}{l}\text { MATa, ade2-101, his3- } \triangle 200, \text { leu2- } \Delta 1, \text { ura3-52, } \\
\text { trp1- } \Delta 63, \text { lys 2-801; tim } 21:: \text { TIM } 21^{\text {ProtA }}-\text { HIS } 3 M X 6\end{array}$ & $\begin{array}{l}\text { (Chacinska et al., } \\
\text { 2005) }\end{array}$ \\
\hline TOM40А & $\begin{array}{l}\text { MATa, ade2-101, his3- } \triangle 200 \text {, leu2- } \Delta 1, \text { ura3-52, } \\
\text { trp1- } 663 \text {, lys } 2-801 ; \text { tom } 40:: A D E 2 ; \text { [pFL39URA3- } \\
\text { TOM40] }\end{array}$ & (Kutik et al., 2008) \\
\hline TOM40 & $\begin{array}{l}\text { MATa, ade2-101, his3- } \triangle 200, \text { leu2- } 1 \text { 1, ura3-52, } \\
\text { trp1- } \triangle 63 \text {, lys2-801; tom } 40:: A D E 2 ; \text { [pFL39TRP1- } \\
\text { TOM40] }\end{array}$ & (Kutik et al., 2008) \\
\hline tom $40^{E \text { mиt }}$ & $\begin{array}{l}\text { MATa, ade } 2-101, \text { his } 3-\triangle 200, \text { leu2- } \Delta 1, \text { ura3-52, } \\
\text { trp1- } \triangle 63 \text {, lys } 2-801 ; \text { tom } 40:: A D E 2 ; \text { [pFL39TRP1- } \\
\text { tom } 40^{E \text { mut }]}\end{array}$ & This study \\
\hline tom $40^{V m u t}$ & $\begin{array}{l}\text { MATa, ade2-101, his3- } \triangle 200, \text { leu2- } \Delta 1, \text { ura3-52, } \\
\text { trp } 1-\triangle 63 \text {, lys } 2-801 ; \text { tom } 40:: A D E 2 ;[\mathrm{pFL} 39 T R P 1- \\
\text { tom } 40^{\text {Vmut }} \text { ] }\end{array}$ & This study \\
\hline
\end{tabular}




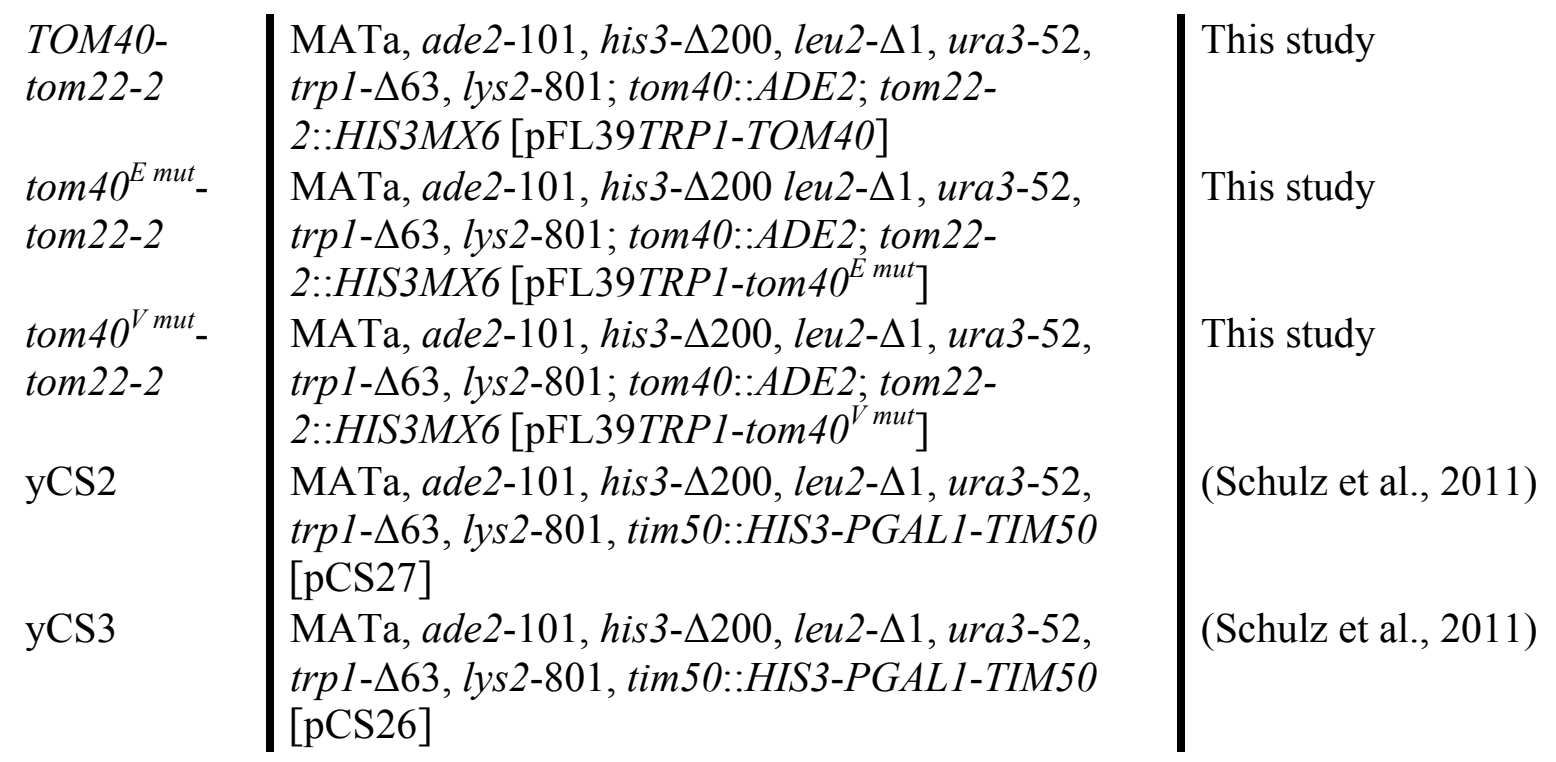

\subsubsection{Laboratory Equipment}

This study was performed using the laboratory equipment listed in Table 9.

Table 9. List of laboratory equipment used throughout the study, along with the corresponding suppler.

Product Supplier

Centrifuges and Rotors

Centrifuge 5417R

Centrifuge 5424

Eppendorf

Centrifuge 5804R

Eppendorf

Rotor F45-30-11

Eppendorf

Rotor F45-24-11

Eppendorf

Eppendorf

Rotor A-4-44

Eppendorf

Sorvall® RC 6TM Plus Superspeed Centrifuge

Sorvall® RC12BPTM Low-Speed Centrifuge

Sorwall H-12000

Rotor Sorwall F14S-6x250Y

Rotor Sorwall F10S-6x500Y

Rotor Sorwall SS-34

Thermo Scientific

Thermo Scientific

Thermo Scientific

Thermo Scientific

Thermo Scientific

Thermo Scientific 


\section{Electrophoresis and Blotting}

EPS 601 power supply

GE-Healthcare
GE-Healthcare
Bio-Rad
Bio-Rad
Bio-Rad
PEQLAB Biotechnologie

FPLC Equipment

ÄKTA Purifier 10

HiLoad 16/60 Superdex 75

HisTrap HP, $1 \mathrm{ml}$ and $5 \mathrm{ml}$

Resource $^{\mathrm{TM}} \mathrm{S} 1 \mathrm{ml}$

GE-Healthcare
GE-Healthcare
GE-Healthcare
GE-Healthcare

\section{Imagining Equipment}

Agfa Curix 60 Developing machine

Autoradiography storage phosphor screen

LAS 3000

Starion FLA-9000

Storm 820 phosphorimager

UVsolo TS transilluminator

AGFA

GE-Healthcare

FujiFilm

FujiFilm

GE-Healthcare

Biometra

Miscellaneous

Autoclave Systec DX-200

Systec

Balance BP 3100P

Sartorius

Electronic Digital Balance Kern ABJ 220-4M

EmulsiFlex C5

Excella ${ }^{\circledR}$ E10 platform shaker

KERN \& Sohn

Avestin

New Brunswick Scientific

G 25 Shaker Incubator

New Brunswick Scientific

iMarkTM Microplate Absorbance Reader

Bio-Rad

Innova ${ }^{\circledR} 44$ Incubator Shaker

GeneQuantTM 1300 Spectrophotometer

New Brunswick Scientific

GE-Healthcare

Magnetic stirrer MR 3001

Heidolph 
Milli-Q-water purification system

NanoVueTM Spectrophotometer

$\mathrm{pH}-\mathrm{meter}$

Pipettes

Potter S Homogenizer

SpeedVac concentrator

Thermomixer Comfort

TPersonal 48 thermocycler

Vortex-Genie 2
Millipore

GE-Healthcare

inoLab

Gilson

Sartorius

Savant

Eppendorf

Biometra

Scientific Industries

\subsubsection{Software}

All software used throughout the course of the study is listed in Table 10.

Table 10. List of software used in the study, along with the corresponding use and developer.

\begin{tabular}{l|l|l} 
Software & Use & Developer \\
\hline & & \\
Adobe Illustrator CS4 & Image processing & Adobe \\
Adobe Photoshop CS4 & Image processing & Adobe \\
DataGraph & Graphing & Visual Data Tools \\
Geneious Pro 4.8.3 & $\begin{array}{l}\text { In silico cloning and mutagenesis } \\
\text { Protein homolog detection }\end{array}$ & Biomatters Ltd. \\
HHpred & $\begin{array}{l}\text { Quantification } \\
\text { ImageQuant TL v 7.0 }\end{array}$ & GE-Healthcare \\
MODELLER & Homology modeling & (Soding et al., 2005) \\
Pymol & Creation of model images & Schrödinger, LLC.
\end{tabular}

\subsubsection{Buffers, Solutions and Media}

All buffers, solutions and culture media used in this work are listed in Table 11 along with their respected composition. 
Table 11. List of buffers and solutions together with their respective composition.

\begin{tabular}{|c|c|}
\hline Buffer or Solution & Composition \\
\hline Acetate buffer & $0.5 \mathrm{M} \mathrm{NH}_{4} \mathrm{CH}_{3} \mathrm{COOH} / \mathrm{CH}_{3} \mathrm{COOH} \mathrm{pH} 3.4$ \\
\hline AVO & $1 \mathrm{mM}$ antimycin $\mathrm{A}, 0.1 \mathrm{mM}$ valinomycin, $2 \mathrm{mM}$ oligomycin \\
\hline \multirow[t]{3}{*}{$\mathrm{b}_{2}$ lysis buffer } & $20 \mathrm{mM}$ MOPS/KOH pH 8.0, $1 \mathrm{mM}$ EDTA, $10 \mathrm{mM}$ \\
\hline & dithiothreitol, $0.1 \%$ Triton X-100, $1 \mathrm{mM}$ PMSF and Complete \\
\hline & EDTA-free protease inhibitor (Roche) \\
\hline $\mathrm{b}_{2}$ buffer $\mathrm{A}$ & $20 \mathrm{mM}$ MOPS/KOH pH 8.0, $1 \mathrm{mM}$ EDTA \\
\hline$b_{2}$ buffer B & $20 \mathrm{mM}$ MOPS/KOH pH 8.0, 1 mM EDTA, $500 \mathrm{mM} \mathrm{NaCl}$ \\
\hline Blotting buffer & $20 \mathrm{mM}$ Tris, $0.02 \%$ SDS, $150 \mathrm{mM}$ glycine, $20 \%$ ethanol \\
\hline BN acrylamide solution & $48 \%$ acrylamide, $1,5 \%$ bisacrylamide $(32: 1)$ \\
\hline BN Anode buffer & $50 \mathrm{mM}$ Bis-Tris/HCl $\mathrm{pH} 7.0$ \\
\hline \multirow[t]{2}{*}{ BN Cathode buffer } & $50 \mathrm{mM}$ tricine, $15 \mathrm{mM}$ Bis-Tris/HCl, with or without $0.02 \%$ \\
\hline & Coomassie Brilliant Blue G250 \\
\hline BN gel buffer $(3 x)$ & $200 \mathrm{mM}$ 6-aminohaxanoic acid, $150 \mathrm{mM}$ Bis-Tris/HCl pH 7.0 \\
\hline \multirow[t]{2}{*}{ BN sample buffer $(10 x)$} & $5 \%$ Coomassie brilliant blue G250, $500 \mathrm{mM}$ 6-aminohaxanoic \\
\hline & acid, 100 mM Bis-Tris/HCl pH 7.0 \\
\hline Carrier DNA & Herring sperm DNA $(10 \mathrm{mg} / \mathrm{ml})$ in TE buffer \\
\hline CNBr Coupling buffer & $100 \mathrm{mM} \mathrm{NaHCO} 3 \mathrm{pH} 8.3,500 \mathrm{mM} \mathrm{NaCl}$ \\
\hline Colloidal Coomassie & $50 \%$ methanol, $2 \%$ phosphoric acid \\
\hline \multicolumn{2}{|l|}{ fixer } \\
\hline Colloidal Coomassie & 0.1\% Coomassie Brilliant Blue G250, $2 \%$ phosphoric acid, $10 \%$ \\
\hline stainer & ammonium sulfate, $20 \%$ methanol \\
\hline Coomassie destainer & $40 \%$ ethanol, $10 \%$ acetic acid \\
\hline \multirow[t]{2}{*}{ Coomassie stainer } & $40 \%$ ethanol, $10 \%$ acetic acid, $0.5 \%$ Coomassie Brilliant Blue \\
\hline & $\mathrm{R} 250$ \\
\hline CSM & $0.67 \%$ yeast nitrogen base, $0.07 \%$ complete synthetic mixture \\
\hline \multirow[t]{2}{*}{ Digitonin buffer } & $20 \mathrm{mM}$ Tris/ $\mathrm{HCl} \mathrm{pH}$ 7.4, $80 \mathrm{mM} \mathrm{NaCl}, 10 \%$ glycerol, $0.5 \mathrm{mM}$ \\
\hline & EDTA, 2 mM PMSF, $1 \%$ digitonin \\
\hline \multirow[t]{2}{*}{ Digitonin wash buffer } & $20 \mathrm{mM}$ Tris/ $\mathrm{HCl} \mathrm{pH}$ 7.4, $80 \mathrm{mM} \mathrm{NaCl}, 10 \%$ glycerol, $5 \mathrm{mM}$ \\
\hline & EDTA, 2 mM PMSF, $0.3 \%$ digitonin \\
\hline
\end{tabular}


DNA loading dye
DTT buffer
5-FOA plates
Glycine elution buffer
Homogenization buffer

Import buffer

\section{LB}

\section{LiAc/PEG}

Protein loading buffer

In vitro pull-down

binding buffer

Resolving buffer (5x)

Rf1 buffer

Rf2 buffer

Running buffer

SEM

SH buffer

Stacking buffer (10x)

TAE buffer

TBS-T

TE

$\operatorname{Tim} 21^{\mathrm{IMS}} / \operatorname{Tim} 23^{\mathrm{IMS}}$

lysis buffer
$10 \%$ sucrose, $0.25 \%$ OrangeG

$10 \mathrm{mM}$ DTT, $100 \mathrm{mM}$ Tris $/ \mathrm{H}_{2} \mathrm{SO}_{4} \mathrm{pH} 9.4$

CSM media, 5.2 mM 5-FOA, $450 \mu \mathrm{M}$ uracil, $2 \%$ sucrose, $2 \%$

agar

$100 \mathrm{mM}$ glycine/HCl pH 2.5

0.6 M sorbitol, $10 \mathrm{mM}$ Tris/ $\mathrm{HCl} \mathrm{pH}$ 7.4, $1 \mathrm{mM}$ EDTA, 0.2\%

fatty acid free BSA, $1 \mathrm{mM}$ PMSF

3\% BSA, $250 \mathrm{mM}$ sucrose, $80 \mathrm{mM} \mathrm{KCl,} 5 \mathrm{mM} \mathrm{MgCl} 2,2 \mathrm{mM}$

KH2PO4, 5 mM methionine, 10 mM MOPS/KOH pH 7.2, 2

$\mathrm{mM}$ ATP, $2 \mathrm{mM}$ NADH, with or without $100 \mu \mathrm{g} / \mathrm{ml}$ creatine

kinase and $5 \mathrm{mM}$ creatine phosphate

$1 \%$ tryptone, $0.5 \%$ yeast extract, $1 \% \mathrm{NaCl}$

$0.1 \mathrm{M}$ lithium acetate, $40 \%$ polyethylene glycol 4000

$10 \%$ glycerol, $2 \%$ SDS, $0.01 \%$ Bromphenol blue, $1 \% \beta$ -

mercaptoethanol, $20 \mathrm{mM}$ Tris/ $\mathrm{HCl} \mathrm{pH} 6.8$

$20 \mathrm{mM}$ HEPES/KOH pH 7.4, $100 \mathrm{mM} \mathrm{KH}{ }_{2} \mathrm{PO}_{4}, 10 \mathrm{mM}$

$\mathrm{Mg}\left(\mathrm{C}_{2} \mathrm{H}_{3} \mathrm{O}_{2}\right)_{2}, 10 \%$ glycerol, $0.5 \%$ Triton X-100

$1.87 \mathrm{M}$ Tris/HCl pH 8.8

$100 \mathrm{mM} \mathrm{RbCl}, 50 \mathrm{mM} \mathrm{MnCl}{ }_{2} \bullet 4 \mathrm{H}_{2} \mathrm{O}, 30 \mathrm{mM} \mathrm{CH} \mathrm{CO}_{2} \mathrm{~K} / \mathrm{C}_{2} \mathrm{H}_{4} \mathrm{O}_{2}$

$\mathrm{pH} 5.8,10 \mathrm{mM} \mathrm{CaCl}_{2} \cdot 2 \mathrm{H}_{2} \mathrm{O}, 15 \%$ glycerol

$10 \mathrm{mM}$ RbCl, $10 \mathrm{mM}$ MOPS/NaOH pH 6.8, $75 \mathrm{mM}$

$\mathrm{CaCl}_{2} \cdot 2 \mathrm{H}_{2} \mathrm{O}, 15 \%$ glycerol

$25 \mathrm{mM}$ Tris, $192 \mathrm{mM}$ glycine, 0.1\% SDS

$250 \mathrm{mM}$ sucrose, $1 \mathrm{mM}$ EDTA, $10 \mathrm{mM}$ MOPS/KOH pH 7.4

0.6 M sorbitol, $20 \mathrm{mM}$ HEPES/NaOH $\mathrm{pH} 7.2$

$0.8 \mathrm{M}$ Tris/ $\mathrm{HCl} \mathrm{pH} 6.8$

$40 \mathrm{mM}$ Tris-acetate $\mathrm{pH}$ 8.0, 2 mM EDTA

$20 \mathrm{mM}$ Tris/ $\mathrm{HCl}$ pH 7.5, $125 \mathrm{mM} \mathrm{NaCl}, 0.1 \%$ Tween 20

$10 \mathrm{mM}$ Tris/ $\mathrm{HCl} \mathrm{pH}$ 8.0, 1 mM EDTA

$50 \mathrm{mM} \mathrm{Na} \mathrm{HPO}_{4} \mathrm{pH}$ 8.0, $300 \mathrm{mM} \mathrm{NaCl}, 5 \mathrm{mM}$ Imidazole, EDTA-free complete protease inhibitor (Roche), 1\% Triton X100 
$\operatorname{Tim} 21^{\text {IMS }} / \operatorname{Tim} 23^{\text {IMS }}$

buffer A

$\operatorname{Tim} 21^{\mathrm{IMS}} / \operatorname{Tim} 23^{\mathrm{IMS}}$

buffer B

$\operatorname{Tim} 21^{\text {IMS }} / \operatorname{Tim} 23^{\text {IMS }}$

dialysis buffer

Tim50 ${ }^{\mathrm{IMS}}$ lysis buffer

$\operatorname{Tim} 50^{\mathrm{IMS}}$ buffer $\mathrm{A}$

$\operatorname{Tim} 50^{\mathrm{IMS}}$ buffer B

Tim50 ${ }^{\text {IMS }}$ dialysis

buffer

Tom22 IP

solubilization buffer

Tom $22^{\text {His }}$ solubilization

buffer (small scale)

Tom $22^{\text {His }}$ solubilization

buffer (large scale)

YP

2x YPAD

Zymolyase buffer
$50 \mathrm{mM} \mathrm{Na}_{2} \mathrm{HPO}_{4} \mathrm{pH} 8.0,300 \mathrm{mM} \mathrm{NaCl}, 5 \mathrm{mM}$ Imidazole

$50 \mathrm{mM} \mathrm{Na}_{2} \mathrm{HPO}_{4} \mathrm{pH}$ 8.0, $300 \mathrm{mM} \mathrm{NaCl}, 500 \mathrm{mM}$ Imidazole

$20 \mathrm{mM}$ HEPES/NaOH pH 7.2, $100 \mathrm{mM} \mathrm{NaCl}$

$20 \mathrm{mM}$ Tris/ $\mathrm{HCl}$ pH 8.0, $500 \mathrm{mM} \mathrm{NaCl}, 15 \mathrm{mM}$ imidazole, $0.1 \%$ Triton $\mathrm{X}-100,1 \mathrm{mM}$ PMSF

$20 \mathrm{mM}$ Tris/ $\mathrm{HCl} \mathrm{pH}$ 8.0, $500 \mathrm{mM} \mathrm{NaCl}, 15 \mathrm{mM}$ imidazole

$20 \mathrm{mM}$ Tris $/ \mathrm{HCl} \mathrm{pH} 8.0,500 \mathrm{mM} \mathrm{NaCl}, 500 \mathrm{mM}$ imidazole

$20 \mathrm{mM}$ Tris/ $\mathrm{HCl} \mathrm{pH} 8.0,500 \mathrm{mM} \mathrm{NaCl}$

$20 \mathrm{mM}$ Tris/ $\mathrm{HCl} \mathrm{pH}$ 7.4, $150 \mathrm{mM} \mathrm{NaCl}, 10 \%$ glycerol, $0.1 \mathrm{mM}$

EDTA, $1 \%$ digitonin, $2 \mathrm{mM}$ pefablock, $2 \mu \mathrm{g} / \mathrm{ml}$ leupeptin and 2 $\mathrm{mM}$ PMSF

$20 \mathrm{mM}$ Tris/ $\mathrm{HCl} \mathrm{pH}$ 7.4, $100 \mathrm{mM} \mathrm{NaCl}, 10 \%$ glycerol, $0.1 \mathrm{mM}$

EDTA, $30 \mathrm{mM}$ imidazole, $1 \%$ digitonin and $1 \mathrm{mM}$ PMSF

$50 \mathrm{mM}$ potassium acetate, $10 \mathrm{mM} \mathrm{MOPS} / \mathrm{KCl} \mathrm{pH} 7.0,20 \%$

glycerol, 1\% digitonin and $1 \mathrm{mM}$ PMSF

$1 \%$ yeast extract, $2 \%$ peptone

$2 \%$ yeast extract, $4 \%$ peptone, $4 \%$ glucose, $0.008 \%$ adenine hemisulphate

1.2 M sorbitol, $20 \mathrm{mM} \mathrm{KH}_{2} \mathrm{PO}_{4} \mathrm{pH} 7.4$

\subsection{E. coli manipulation}

\subsubsection{Preparation of chemically competent $E$. coli}

XL1-Blue and BL21 (Stratagene) E. coli strains were initially grown overnight in LB media at $37^{\circ} \mathrm{C}$ with vigorous shaking after inoculation from cryo-stock ( $20 \%$ glycerol at $-80^{\circ} \mathrm{C}$ ). The cells were made competent via the $\mathrm{RbCl}$ method according to the following protocol. Cells from the overnight culture were used to inoculate a fresh culture to 1:500 and cells were returned to $37^{\circ} \mathrm{C}$ under aeration till an $\mathrm{OD}_{600}$ value of 0.5 was reached. At that point, cells were chilled on ice for $15 \mathrm{~min}$ and centrifuged at 1,000 xg for $10 \mathrm{~min}$ at 
$4^{\circ} \mathrm{C}$. After centrifugation, the supernatant was removed and pre-cooled Rf1 buffer was used to resuspend the cell pellet, after which the cells were incubated on ice for $15 \mathrm{~min}$. Next, the cells were again pelleted as previously stated and then resuspended in pre-cooled Rf2 buffer, prior to aliquoting and flash freezing in liquid $\mathrm{N}_{2}$. After which, the cells were stored at $-80^{\circ} \mathrm{C}$ until use.

\subsubsection{Transformation of chemically competent $E$. coli}

Chemically competent E. coli (either XL1-Blue or BL21 [Stratagene]) were removed from $-80^{\circ} \mathrm{C}$ and thawed gently on ice. Subsequently, $50-200 \mathrm{ng}$ of plasmid DNA was added and the mixture as incubated for $10 \mathrm{~min}$ on ice. Following incubation, the cell mixture was heat shocked for 45 seconds at $42^{\circ} \mathrm{C}$ under gentle agitation. After heat shock, cells were left to recover for 2 min on ice and then ample LB media was added to the container. Cells in LB media were then shifted to $37^{\circ} \mathrm{C}$ and grown for $1 \mathrm{hr}$ under strong agitation. After the $37^{\circ} \mathrm{C}$ incubation, cells were pelleted lightly at $1,000 \mathrm{xg}$ and plated on solid LB media (LB plus $1.5 \%$ agar) containing ampicillin $100 \mathrm{mg} / \mathrm{ml}$ and grown at $37^{\circ} \mathrm{C}$ until single colony selection was possible.

\subsection{Molecular Biology}

\subsubsection{Plasmid isolation from XL-1 Blue $E$. coli}

Approximately $8 \mathrm{ml}$ of an overnight XL-1 Blue culture was pelleted and subjected to DNA isolation using the Wizard ${ }^{\circledR}$ Plus SV Minipreps DNA Purification system (Promega) according to the manufacture's instructions. Isolated DNA was quantified using a NanoVue spectrophotometer (GE-Healthcare), monitoring the absorbance at $260 \mathrm{~nm}$. DNA was then frozen at $-20^{\circ} \mathrm{C}$ until use.

\subsubsection{Polymerase chain reaction (PCR)}

DNA amplification via PCR was performed using a KOD Hot Start DNA polymerase kit (Novagen) according to the manufactures instructions. Each reaction contained 100 - $200 \mathrm{ng}$ of template DNA, $1.5 \mathrm{mM} \mathrm{MgSO}_{4}, 0.2 \mathrm{mM}$ dNTP mix, $0.3 \mu \mathrm{M}$ forward and reverse primers, $1 \mathrm{x}$ KOD buffer and 0.02 units/ $\mu 1 \mathrm{KOD}$ polymerase. The PCR cycling program was as follows: 2 min KOD activation $95^{\circ} \mathrm{C}$, followed by 35 cycles of denaturation for $20 \mathrm{sec}$ at $95^{\circ} \mathrm{C}$, annealing for $10 \mathrm{sec}$ at the lowest primer melting 
temperature and elongation for $20 \mathrm{sec}$ each $\mathrm{kb}$ of product. After the cycling iterations were complete, the reaction was subjected to agarose gel electrophoresis with ethidium bromide staining. For the creation of the integration cassette used to create the tom22-2 allele (Frazier et al., 2003), the following primers were used with pYM10 as the template: AEF44 (5' - GAC CAC CAC TGC TTT GTT ACT CGG TGT GCC ACT ATC CTT ATC TAT ACT TGC CGA ACA ATA GGG CGC GCC ACT TCT A - 3') and AEF45 (5' CAT GTA TGG CTC CTT TTC TAA AAC CCT CTC TTT TCT TTT ACA TCA TTA AAA TTA ATG GCA TCG ATG AAT TCG AGC TCG - 3’').

\subsubsection{Agarose gel electrophoresis}

Separation of DNA was achieved via agarose gel electrophoresis. Agarose gels were prepared by dissolving $1 \%$ agarose in TAE buffer, to which $1 \mu \mathrm{g} / \mathrm{ml}$ ethidium bromide was added, prior to gel casting. After DNA was mixed with the DNA loading dye, the gel was run at $8 \mathrm{~V} / \mathrm{cm}$ in a MINI-Sub ${ }^{\circledR}$ Cell GT Cell (Bio-Rad) filled with TAE buffer. Following the gel run, fragments were visualized through the use of a UVsolo TS transilluminator (Biometra). If the downstream application required, the approprate DNA band was excised from the gel for subsequent isolation using the Wizard ${ }^{\circledR} \mathrm{SV}$ Gel and PCR Clean-up system (Promega) according to the manufacture's instructions.

\subsubsection{Site-directed mutagenesis}

Site-directed mutagenesis was preformed using the QuikChange ${ }^{\circledR}$ Lightning kit (Stratagene) according to the manufacture's instructions in order to create the point mutants needed for the study. The following primers were used: Tom40 ${ }^{\mathrm{T} 220 \mathrm{E}} \mathrm{JM} 26$ (5' ATACTCCAGAGAAGACGGTAGCGCTCCAG - 3') and JM27 (5' TACCGTCTTCTCTGGaGtATAAAGTTTCTA - 3'); $\quad$ Tom40 $1220 \mathrm{~V} \quad$ JM28 $\quad\left(5^{\prime}-\right.$ ATACTCCAGAGTTGACGGTAGCGCTCCAG - 3') and JM29 (5' TACCGTCAACTCTGGAGTATAAAGTTTCTA - 3'). 50 ng of template DNA (Tom40Trp) was mixed with $125 \mathrm{ng}$ of each primer pair in the $50 \mu \mathrm{l}$ reaction as specified in the manufacture's instructions. The reaction was performed with the following steps: polymerase activation at $95^{\circ} \mathrm{C}$ for $30 \mathrm{sec}$ and 20 cycles $\left(95^{\circ} \mathrm{C}\right.$ for $30 \mathrm{sec}, 1 \mathrm{~min}$ primer annealing at $48^{\circ} \mathrm{C}$ and 6 min extension at $68^{\circ} \mathrm{C}$ ). After completion, $2 \mu$ of Dpn1 was added to the reaction and incubated for $1 \mathrm{hr}$ at $37^{\circ} \mathrm{C}$ in order to digest the non-methylated template DNA. Subsequently, $12 \mu \mathrm{l}$ of the reaction was added to $50 \mu 1$ of the supplied ultra 
competent XL-1 Blue cells and the transformation was performed according to the manufacture's instructions. Following transformation, all cells were plated on solid LB media containing ampicillin as stated in section 2.2.2. and DNA was isolated from a single colony as specified in section 2.3.1.

\subsubsection{Sequencing of DNA constructs}

DNA constructs were sent to GATC Biotech (Cologne) where the sequencing reaction was performed, followed by sequencing using a Sanger ABI 3730xl platform. After receipt of the sequencing data file, the sequencing reads were analyzed through the use of Geneious Pro 5.3.6 (Kearse et al., 2012).

\subsubsection{Peptide synthesis}

Presequence probes were synthesized identically to the previously published protocol (Schulz et al., 2011), through the use of a peptide synthesizer (Applied Biosystems) via standard fluorenylmethoxycarbonyl chemistry. The UV activatable amino acid derivative para-benzoyl-Phe-OH (BPA [Bachem]) was added to the polypeptide chain through the use of an Fmoc derivative, and the biotin tag was introduced through the use of a biotinylated Lys residue. Following synthesis completion, peptides were severed from their resin and deprotected with 95\% TFA, 2.5\% triisopropylsilane and 2.5\% $\mathrm{H}_{2} \mathrm{O}$ for $4 \mathrm{hr}$ at room temperature. Peptides removed from the resin were characterized via reversedphase HPLC and mass spectrometry. N-terminal amine and C-terminal amide pCox 4 and Synb2 were purchased from JPT Peptide Technologies. All peptides were resuspended in $10 \mathrm{mM}$ acetic acid, aliquoted and stored at $-20^{\circ} \mathrm{C}$ until use.

\subsection{S. cerevisiae methods}

\subsubsection{S. cerevisiae growth conditions}

S. cerevisiae were grown in both liquid and solid (addition of 2\% agar) YP media supplemented with 3\% glycerol unless plasmid selection was required. When YP media was used, it was set to $\mathrm{pH} 5.8$ with $\mathrm{HCl}$ before being autoclaved. In these cases, CSM media (lacking the appropriate component[s]) supplemented with $2 \%$ sucrose was utilized. Long-term storage of yeast was done at $-80^{\circ} \mathrm{C}$ in media containing $2 \mathrm{xYPAD}$ and $20 \%$ glycerol. 
Dilution growth tests on solid media were performed via $\mathrm{OD}_{600}$ normalization of an overnight YP culture, supplemented with 3\% glycerol. Cells were spotted on plates containing YP media containing 3\% glycerol in 10 fold serial dilutions and grown for three days at the specified temperature, at which point an image of the plate was captured with an LAS 3000.

\subsubsection{5-FOA plasmid shuffling}

S. cerevisiae containing multiple plasmids, encoding selection markers URA3 and TRP1, were first grown on CSM plates lacking both uracil and tryptophan containing 2\% sucrose. Shuffling was achieved through plating on 5-FOA containing solid media, which were shielded from light at $30^{\circ} \mathrm{C}$ for three days. After initial plasmid selection, yeast were applied to an additional round of 5-FOA based plasmid selection before being placed back on plates containing YP supplemented with 3\% glycerol.

\subsubsection{Transformation of $S$. cerevisiae}

Transformation of either plasmids or integration cassettes was done using the lithium acetate/PEG method previously established (Gietz and Schiestl, 2007), with subtle modifications. The appropriate yeast strain was grown overnight in 2xYPAD media and then reinoculated the following morning to an $\mathrm{OD}_{600}$ reading of 0.25 . After the culture reached an $\mathrm{OD}_{600}$ reading of 2 , the cells were pelleted via centrifugation at 3,000 $\mathrm{xg}$ for 10 min. Subsequently, cells were washed with sterile water and then resuspended in $0.1 \mathrm{M}$ lithium acetate. Following lithium acetate incubation, cells were either aliquoted and frozen at $-80^{\circ} \mathrm{C}$ or the transformation protocol was continued. $120 \mu \mathrm{g}$ of herring sperm DNA was denatured at $95^{\circ} \mathrm{C}$ for $5 \mathrm{~min}$ and rapidly cooled on ice, then added to $100 \mu \mathrm{l}$ of lithium acetate competent yeast, along with $200 \mathrm{ng}$ plasmid DNA or $1 \mu \mathrm{g}$ of integration cassette. With every transformation a corresponding control was performed in which a similar volume of sterile water was added instead of DNA. Immediately following DNA addition, $600 \mu \mathrm{l}$ of LiAc/PEG buffer was applied and the mixture was incubated for $1 \mathrm{hr}$ at $30^{\circ} \mathrm{C}$ under heavy agitation. After incubation, $68 \mu \mathrm{l}$ of DMSO was added and the transformation mixture was placed at $42^{\circ} \mathrm{C}$ for 15 min under strong aeration. After heatshock, the cells were pelleted gently at 1,000 xg and the supernatant removed. The cells were resuspended in $60 \mu \mathrm{l}$ of $1 \mathrm{M}$ sorbitol, plated on the corresponding solid selection media and grown for $2-3$ days at $30^{\circ} \mathrm{C}$. Single colonies were transferred to another plate 
for a second round of selection. Cells were then examined at the protein level for conformation of genetic alteration.

\subsubsection{Subcellular fraction of yeast, mitochondrial isolation}

Mitochondria were isolated from yeast through a differential centrifugation protocol as previously published (Meisinger et al., 2006). Yeast were cultured in YP media containing $3 \%$ glycerol overnight to an $\mathrm{OD}_{600}$ reading of $1-3$. Cell pellets were obtained via centrifugation $7,000 \mathrm{xg}$ for $15 \mathrm{~min}$ and washed with water. Afterwards, cells were resuspended in DTT buffer and kept under mild agitation for $30 \mathrm{~min}$ at $30^{\circ} \mathrm{C}$. Following DTT treatment, cells were recollected, washed with 1.2 M sorbitol and then placed in zymolyase buffer ( $7 \mathrm{ml}$ per g yeast pellet) to which $4 \mathrm{mg}$ of zymolyase was added per $\mathrm{g}$ of yeast pellet. Zymolyase treatment was performed for $1 \mathrm{hr}$ at $30^{\circ} \mathrm{C}$ under gentle agitation. Spheroplasts were then washed with zymolyase buffer prior to resuspension in pre-cooled homogenization buffer. The plasma membrane was opened through 20 strokes of a glass Potter S dounce homogenizer set at $800 \mathrm{rpm}$. Differential centrifugation then commenced with the removal of unopened and large cell debris by an initial step at 2,000 xg for $5 \mathrm{~min}$. The resulting supernatant was subjected to an additional cleaning step at 7,000 $\mathrm{xg}$ for 10 mins, prior to spinning down the mitochondrial fraction at $17,000 \mathrm{xg}$ for $15 \mathrm{~min}$. The mitochondrial fraction pellet was pooled and washed with SEM buffer containing $1 \mathrm{mM}$ PMSF. The mitochondrial fraction was analyzed via a Bradford assay enabling the normalization of each strain through total protein levels. Protein determination was performed with Roti-Quant reagent and a bovine $\operatorname{IgG}$ as a standard at concentrations 7.5, 15,30 and $60 \mu \mathrm{g} / \mu \mathrm{l}$. Three different mitochondrial fraction concentrations were utilized to increase protein determination accuracy. After measuring the absorbance at $595 \mathrm{~nm}$, mitochondria isolated from various yeast strains were normalized to $10 \mu \mathrm{g} / \mu \mathrm{l}$ and aliquoted prior to flash freezing in liquid $\mathrm{N}_{2}$ and storage at $-80^{\circ} \mathrm{C}$.

\subsection{Purification of recombinant proteins from $E$. coli}

\subsubsection{BL21 E coli protein expression}

The BL21 E. coli strain with the protein of interest under the control of the lacZ promoter was used for the expression of recombinant proteins. An overnight LB ampicillin culture was used to inoculate a fresh $\mathrm{LB}$ ampicillin culture to an $\mathrm{OD}_{600}$ value of 0.015 , and 
then placed at $37^{\circ} \mathrm{C}$ under heavy aeration until an $\mathrm{OD}_{600}$ reading of 0.8 was reached. $1 \mathrm{mM}$ IPTG was used for induction and the cells were grown for another $2-4 \mathrm{hr}(2 \mathrm{hr}$ for $b_{2}(167)_{A}$-DHFR and $4 \mathrm{hr}$ for all others). Cells were then collected at 7,000 xg for $15 \mathrm{~min}$ and frozen at $-20^{\circ} \mathrm{C}$ until needed for purification.

\subsubsection{Ion exchange chromatography}

$\mathrm{b}_{2}(167)$-DHFR was purified through ion exchange chromatography on an ÄKTA FPLC system using a Resource S $1 \mathrm{ml}$ column similarly to a previously published protocol (Dekker et al., 1997). Frozen cells pellets of expressed $b_{2}(167)_{4}$-DHFR were defrosted and resuspended in $\mathrm{b}_{2}$ lysis buffer containing DNase I (1mg per $5 \mathrm{~g} \mathrm{E}$. coli wet weight) and passed through an EmulsiFlex C5 at 1,000 bar thrice. Lysed cells were pelleted to remove all non-soluble entities at 20,000 $\mathrm{xg}$ and the supernatant was passed through a $0.2 \mu \mathrm{M}$ cutoff syringe filter. Filtered cell lysate was then loaded on a Resource S column using an ÄKTA FPLC system set at $0.5 \mathrm{ml} / \mathrm{min}$ with $\mathrm{b}_{2}$ buffer A. After loading, the column was washed with $10 \mathrm{ml}$ of $b_{2}$ buffer $A$ at $1 \mathrm{ml} / \mathrm{min}$, allowing for the absorbance at $280 \mathrm{~nm}$ of the buffer passing over the column to return to a baseline value. After washing, bound proteins were eluted at $1 \mathrm{ml} / \mathrm{min}$ using $\mathrm{b}_{2}$ buffer B via a linear gradient from $0-100 \%$ buffer $\mathrm{B}$ in 10 column volumes. Fractions collected corresponding to the highest absorbance values at $280 \mathrm{~nm}$ were pooled, aliquoted, flash frozen with liquid $\mathrm{N}_{2}$ and stored at $-80^{\circ} \mathrm{C}$.

\subsubsection{Metal affinity chromatography of recombinant proteins}

E. coli expressed recombinant proteins containing a histidine tag (Tim50 ${ }^{\mathrm{IMS}}$, $\operatorname{Tim} 21^{\mathrm{IMS}}, \operatorname{Tim} 23^{\mathrm{IMS}}$, and Tim2 $3^{\mathrm{YL} 70 \mathrm{AA}}$ ) were purified using a 1 or $5 \mathrm{ml}$ HisTrap HP column, mounted on an ÄTKA FPLC system. Frozen cell pellets were lysed, centrifugally clarified and filtered as in section 2.5.2., utilizing either $\operatorname{Tim} 21^{\mathrm{IMS}} / \operatorname{Tim} 23^{\mathrm{IMS}}$ lysis buffer or $\operatorname{Tim} 50^{\mathrm{IMS}}$ lysis buffer for the respective proteins. Loading on the HisTrap HP column was performed at $0.5 \mathrm{ml} / \mathrm{min}$, after which the column was washed with either $\operatorname{Tim} 21^{\mathrm{IMS}} / \operatorname{Tim} 23^{\mathrm{IMS}}$ buffer A or Tim $50^{\mathrm{IMS}}$ buffer $\mathrm{A}$ as in section 2.5.2. When a stable absorbance value at $280 \mathrm{~nm}$ was achieved (normally $10-20$ column volumes of washing were required), bound proteins were eluted with a linear gradient of increasing corresponding buffer B, containing $500 \mathrm{mM}$ imidazole at a flow rate of $1 \mathrm{ml} / \mathrm{min}$. Peak 280 $\mathrm{nm}$ absorbance fractions were analyzed via SDS-PAGE and Coomassie Brilliant Blue 
R250 staining (see sections 2.6.1. and 2.6.3. for a detailed descriptions). The cleanest fractions were pooled and dialyzed over night in the appropriate buffer, after which the protein was concentrated with centrifugal filters. Following concentration, a Bradford protein determination assay was preformed using Roti-Quant reagent and BSA as a standard at 2, 4, 8, 12 and $16 \mu \mathrm{g} / \mu \mathrm{l}$. The absorbance values at $595 \mathrm{~nm}$ were read in duplicate in a plate reader using a 96 well plate. Following the ascertainment of the concentration, small aliquots were made, flash frozen in liquid $\mathrm{N}_{2}$ and stored at $-80^{\circ} \mathrm{C}$.

\subsubsection{Size exclusion chromatography}

Gel filtration was performed using an ÄKTA FPLC system as a cleaning step whenever purity was a problem following HisTrap purification. Depending on the size of the recombinant protein and its intrinsic ability to form higher oligomers, either a HiLoad 16/60 Superdex 200 or 75 was used. Before each run, at least 1.5 column volumes of the appropriate buffer were used to equilibrate the column before the protein sample was loaded. At a flow rate of $1 \mathrm{ml} / \mathrm{min}$, proteins were separated and fractions were collected, which were later examined as in section 2.5.3.

\subsection{PAGE protein analysis}

\subsubsection{SDS-PAGE}

SDS-PAGE was performed essentially as previously published (Laemmli, 1970) using acrylamide (37.5:1 acrylamide:bisacrylamide). A 4\% acrylamide gel containing $0.1 \%$ SDS and $1 \mathrm{x}$ stacking buffer as a stacking gel, combined with a resolving gel containing $10-15 \%$ acrylamide, $1 \times$ resolving buffer and $0.1 \%$ SDS was used for SDSPAGE. Both sections of the gel were polymerized with $0.1 \%$ ammonium peroxodisulphate and $0.05 \%$ TEMED. Before proteins were applied to the gel, they were mixed with $4 \mathrm{x}$ protein loading buffer to a final concentration of $1 \mathrm{x}$ and placed at $95^{\circ} \mathrm{C}$ for $5 \mathrm{~min}$. Gels were either run in a Mini-PROTEAN ${ }^{\circledR}$ Cell (Bio-Rad) or in a custom-made running chamber at $20 \mathrm{~mA} / \mathrm{gel}$ or $30 \mathrm{~mA} / \mathrm{gel}$, respectively.

\subsubsection{BN-PAGE}

BN-PAGE was used to resolve native protein complexes and performed essentially as previously published (Schägger and Jagow, 1991). Solubilized mitochondria were 
mixed with 10x BN sample buffer to a final concentration of 1x. Samples were loaded on a $4-13 \%$ (for non-radioactive samples) or a $6-16.5 \%$ gradient gel, cast with a custommade gradient mixer, driven by a peristaltic pump. The gel solutions contained the appropriate amount of $\mathrm{BN}$ acrylamide solution, $1 \mathrm{x} \mathrm{BN}$ gel buffer and with the higher percentage receiving $20 \%$ glycerol. After the gradient gel was cast, a $4 \%$ stacking gel was added, containing 1x BN gel buffer. Gels were run at $600 \mathrm{~V}$ and $15 \mathrm{~mA} / \mathrm{gel}$ on a Hoefer SE600 Ruby Blue native system at $4^{\circ} \mathrm{C}$. For gels which needed to be blotted, the cathode buffer was exchanged with cathode buffer without Coomassie Brilliant Blue G250 after the sample had entered the resolving section of the gel.

\subsubsection{Coomassie Brilliant Blue staining}

Both polyacrylamide gels as well as PVDF membrane-containing blotted proteins were subjected to Coomassie Brilliant Blue staining. Coomassie stainer solution was incubated with gels for $60 \mathrm{~min}$ and with membranes for $1 \mathrm{~min}$. Following staining incubation time, membranes were washed with Coomassie destainer solution for $5 \mathrm{~min}$, followed by a 5 min wash in $100 \%$ methanol to completely remove the Coomassie before commencing with Western-blotting (see section 2.6.5. for details). Gels were washed with Coomassie destainer thrice for $30 \mathrm{~min}$ to remove non-specific Coomassie staining.

\subsubsection{Colloidal Coomassie staining}

Protein staining of gels intended for mass spectrometric analysis was done through colloidal Coomassie staining according to a previously published protocol (Neuhoff et al., 1988). After electrophoresis, gels were washed with water prior to incubation in colloidal Coomassie fixer for $1 \mathrm{hr}$. Following fixation, gels were washed twice with water and placed in colloidal Coomassie stainer overnight or until an acceptable level of staining had been achieved. After staining, residual Coomassie was removed with $1 \%$ acetic acid and then placed in water until needed for mass spectrometric analysis (see section 2.6.7. for details).

\subsubsection{Western-blotting and immunolabeling of PVDF membrane}

Following SDS- and BN-PAGE, gels were transferred to PVDF membrane through a semi-dry blotting method in PEQLAB chambers. PVDF membrane was activated with $100 \%$ methanol and then placed in blotting buffer. Three layers of Whatman paper, which had been previously submerged in blotting buffer was placed on the lower electrode of the 
chamber. The activated and blotting buffer equilibrated PVDF membrane was placed atop of the Whatman paper, followed by the polyacrylamide gel and three additional layers of blotting buffer soaked Whatman paper. Before the blotting chamber was closed, all air bubbles trapped in between the layers was removed. After sealing the blotting chamber, $250 \mathrm{~mA}$ was applied for $2 \mathrm{hr}$.

After the transfer of proteins to the PVDF membrane, the membrane was stained as described in section 2.6.3. and then incubated in 5\% milk powder dissolved in TBS-T for 1 $\mathrm{hr}$ at room temperature or overnight at $4^{\circ} \mathrm{C}$. Following blocking, membranes were shortly washed twice with TBS-T prior to incubation in primary antibody for $1 \mathrm{hr}$ at room temperature. Membranes were again washed thrice with TBS-T after primary antibody incubation for $30 \mathrm{~min}$, after which they were placed in the appropriate secondary antibody for $1 \mathrm{hr}$ at room temperature. Immediately prior to signal detection, membranes were washed with ample amounts of TBS-T three times for $30 \mathrm{~min}$. Membranes incubated in HRP coupled secondary antibody were incubated in enhanced chemiluminescence detection reagent and then placed inside of a developing cassette, enabling the exposure medical x-ray films to the membranes. Following exposure, films were inserted into an Agfa Curix 60 Developing machine. Moreover, membranes were also imaged through the use of an LAS 3000 when necessary. Finally, membranes which had been incubated with a fluorophore coupled secondary antibody were washed three times for $5 \mathrm{~min}$ and then placed in a Starion FLA-9000 scanner, where the fluorescently generated signals were imaged.

\subsubsection{Digital autoradiography}

Radioactive gels were imaged via digital autoradiography on a Storm 820 phosphorimager. Polyacrylamide gels were first dried and adhered at a sheet of Whatman paper under vacuum at $65^{\circ} \mathrm{C}$. Gels were placed under an autoradiography storage phosphor screen and exposed for various periods of time before being imaged on a phosphorimager. After the image file was generated, key signals were quantified through the use of ImageQuant TL v 7.0.

\subsubsection{Mass spectrometric analysis of proteins}

Colloidal Coomassie stained proteins in SDS-PAGE gels were identified and analyzed via MALDI nano LC-MS/MS essentially as previously published (Jahn et al., 2002; Rodríguez-Castañeda et al., 2010; Schulz et al., 2011). First, stained protein bands 
were excised from the gel and subjected to in-gel tryptic digestion. Gel pieces were incubated in a series of solutions: (i) $25 \mathrm{mM} \mathrm{NH}_{4} \mathrm{HCO}_{3} / \mathrm{H}_{2} \mathrm{O}$; (ii) $25 \mathrm{mM} \mathrm{NH} \mathrm{HCO}_{3} / 50 \%$ acetonitrile; (iii) $100 \%$ acetonitrile; (iv) reduction in $10 \mathrm{mM}$ dithiothreitol, $25 \mathrm{mM}$ $\mathrm{NH}_{4} \mathrm{HCO}_{3} / \mathrm{H}_{2} \mathrm{O}$ at $56^{\circ} \mathrm{C}$ for $1 \mathrm{hr}$; (v) steps $\mathrm{i}$ - iii repeated; (vi) carbamidomethylation in 25 $\mathrm{mM}$ indoacetamide, $25 \mathrm{mM} \mathrm{NH} \mathrm{HCO}_{3} / \mathrm{H}_{2} \mathrm{O}$. Digestion was performed with $120 \mathrm{ng}$ of trypsin at $37^{\circ} \mathrm{C}$ overnight. The next morning, peptides were extracted with $1 \%$ trifluoroacetic acid (TFA), dried in a SpeedVac concentrator, resuspended in 9.5\% acetonitrile with $0.1 \%$ TFA and subjected to reversed-phase chromatography on a PepMap100 C18 nano-column (Dionex), mounted on a EASY-nLC (Bruker Daltonics) with $9.5 \%-90.5 \%$ acetonitrile in $0.1 \%$ TFA in 80 min. Peptides eluting from the column were mixed with matrix solution $(4.5 \%$ saturated $\alpha$-cyano-4-hydroxycinnamic acid dissolved in $90 \%$ acetonitrile, $0.1 \%$ TFA and $1 \mathrm{mM} \mathrm{NH}_{4} \mathrm{H}_{2} \mathrm{PO}_{4}$ ) and spotted on an anchorchip through the use of a Proteineer fc II (Bruker Daltonics). Spotted samples were investigated on the chip using an Ultraflextreme (Bruker Daltonics) mass spectrometer analyzing MS and post-source decay MS/MS spectra supported with WARP-LC, AutoXecute, Flex-Analysis and Biotools software (Bruker Daltonics). Moreover, when photo-crosslinking MS/MS based analyses were performed, all software detected potential crosslink-containing spectra were reevaluated manually for confirmation.

\subsection{In organello mitochondrial assays}

\subsubsection{In vitro transcription/translation and ${ }^{35} \mathrm{~S}$ labeling of proteins}

${ }^{35} \mathrm{~S}$ labeled proteins were obtained through the coupled transcription/translation in a rabbit reticulocyte lysate kit. Plasmid DNA, encoding the protein of interest under an SP6 promoter, was mixed with reticulocyte lysate master mix $(1 \mu \mathrm{g}$ DNA/50 $\mu 1$ reaction volume) and $\left[{ }^{35} \mathrm{~S}\right]$ methionine $(100 \mu \mathrm{C} / 100 \mu \mathrm{l})$, incubated at $30^{\circ} \mathrm{C}$ for $1.5 \mathrm{hr}$ under mild agitation. Following the transcription/translation reaction, the synthesis was quenched with $50 \mathrm{mM}$ cold methionine, prior to the addition of $300 \mathrm{mM}$ sucrose to provide osmotic support in downstream mitochondrial applications. The lysate was analyzed via SDSPAGE and digital autoradiography (for details, see sections 2.6.1. and 2.6.6., respectively) for the presences of a specific radioactive signal, prior to being flash frozen in liquid $\mathrm{N}_{2}$ and stored at $-80^{\circ} \mathrm{C}$ until use. 


\subsubsection{In vitro import into isolated mitochondria}

Both radiolabeled and recombinant proteins were imported into isolated mitochondria essentially following the previously published protocol (Wiedemann et al., 2006). After mitochondria were gently thawed on ice, $75 \mu \mathrm{g}$ of mitochondrial protein fraction were resuspended in $100 \mu \mathrm{l}$ of import buffer, to which either $1 \%$ AVO mix (when depletion of the $\Delta \psi$ was required) or ethanol (the corresponding solvent) was added. In the case of import times over $20 \mathrm{~min}$, creatine kinase and creatine phosphate were added to the import buffer. The import reaction was pre-heated at $25^{\circ} \mathrm{C}$ for $3 \mathrm{~min}$ before the reaction was started. Radiolabeled substrates were added at $5-10 \%$ of the import reaction and $15 \%$ for non-radiolabeled substrates. After the specified import time, 1\% AVO mix was used to stop import and the reaction was placed on ice where it was Proteinase $\mathrm{K}$ treated $(7 \mu \mathrm{g} / 100$ $\mu 1$ reaction) for $10 \mathrm{~min}$ on ice, with the exception of the Oxa1 ${ }^{\text {GIP }}$ OM translocation assay, which received no Proteinase K. After the digestion of all non-imported substrate, $2 \mathrm{mM}$ PMSF was added and the reaction was incubated for 15 min on ice. After, mitochondria were pelleted at $20,000 \mathrm{xg}$ for $10 \mathrm{~min}$ at $4^{\circ} \mathrm{C}$ and washed with ample SEM buffer to remove BSA from the mitochondrial pellet. Samples to be analyzed via SDS-PAGE were resuspended in protein loading buffer supplemented with $2 \mathrm{mM}$ PMSF and incubated at $95^{\circ} \mathrm{C}$ for $10 \mathrm{~min}$. Samples intended for BN-PAGE were resuspended in digitonin buffer and treated as described in section 2.7.3.

\subsubsection{Mitochondrial membrane solubilization}

Mitochondria were prepared for BN-PAGE through digitonin solubilization closely following a previously published procedure (Dekker et al., 1997). The mitochondrial pellet obtained via centrifugation at $20,000 \mathrm{xg}$ for $10 \mathrm{~min}$ at $4{ }^{\circ} \mathrm{C}$ was resuspended in digitonin buffer through gentle pipetting (20 iterations) and left on ice for $20 \mathrm{~min}$. Following membrane solubilization, the lysate was clarified through centrfugation for $10 \mathrm{~min}$ at $20,000 \mathrm{xg}, 4^{\circ} \mathrm{C}$. After clarification, the supernatent was removed, mixed with $10 \mathrm{x} \mathrm{BN}$ sample buffer to a final concentration of $1 \mathrm{x}$ and subjected to BN-PAGE as described in section 2.6.2.

\subsubsection{In organello crosslinking}

In organello photo-crosslinking was performed essentially as previously published (Schulz et al., 2011). Mitochondria were defrosted gently on ice and added to import buffer 
lacking BSA for a final mitochondrial protein concentration of $1 \mu \mathrm{g} / \mu 1$. Photo-crosslinking reactions in Tom40 mutant mitochondria contained an additional $75 \mathrm{mM} \mathrm{NaCl}$. The presequence probe, or the corresponding solvent (10 $\mathrm{mM}$ actetic acid), was added to the reaction at $2 \mu \mathrm{M}$ and incubated for $10 \mathrm{~min}$ on ice, shielded from ambient light. Following the initial incubation on ice, the reactions were subjected to UV irradiation for $30 \mathrm{~min}$ on ice under a custom-made halogen metal vapor lamp with a glass screen filtering-out protein damaging wavelengths below $300 \mathrm{~nm}$. Photo-crosslinking was performed in $1.5 \mathrm{ml}$ mirco tubes containing no more than $100 \mu$ to ensure sufficient UV penetration into the aqueous reaction. After UV irradiation, mitochondria were pelleted at $20,000 \mathrm{xg}$ at $4^{\circ} \mathrm{C}$ for 10 min and washed with SEM buffer prior to the addition of protein loading buffer and incubation at $95^{\circ} \mathrm{C}$ for $10 \mathrm{~min}$ and SDS-PAGE analysis (see section 2.6.1. for a detailed description).

\subsubsection{Immunoprecipitation}

Immunoprecipitation was performed with rabbit polyclonal antisera directed against Tom22, Tim50 or Tim 23 crosslinked to proteinA-sepharose ( $4 \mu 1$ serum/ $\mu 1$ resin). HA immunoprecipitation was performed using mouse monoclonal (12CA5) hybridoma supernatant crosslinked to proteinG-sepharose ( $8 \mu \mathrm{l}$ supernatant $/ \mu 1$ resin). IgG was bound to the resin according to the manufacture's instructions (GE-Healthcare) in $100 \mathrm{mM}$ $\mathrm{KH}_{2} \mathrm{PO}_{4} \mathrm{pH} 7.4$ and immunoprecipitations were carried-out as previously published (Lytovchenko et al., 2013). Following serum or hybridoma supernatant incubation with either proteinA- or G-sepharose, DMP crosslinking was performed for $30 \mathrm{~min}$ at room temperature $(6 \mathrm{mg} / \mathrm{ml})$ in $100 \mathrm{mM} \mathrm{Na}$ borate/ $\mathrm{NaOH}$ pH 9.0. Quenching was achieved with $100 \mathrm{mM}$ Tris $/ \mathrm{HCl} \mathrm{pH} 7.4$ for either $1 \mathrm{hr}$ at room temperature or overnight at $4{ }^{\circ} \mathrm{C}$. Quenched, IgG bound resin was washed 3x with ample acetate buffer and the $\mathrm{pH}$ was

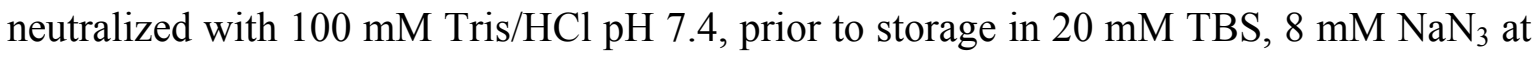
$4^{\circ} \mathrm{C}$ until use.

For Tim50, Tim 23 and HA immunoprecipitations, mitochondria were resuspended in digitonin buffer, lacking digitonin, containing $50 \mu \mathrm{M}$ pCox 4 or Synb2 and incubated on ice for 10 min prior to solubilization by the addition in 5\% digitonin (as in section 2.7.3.), to a final digitonin concentration of $1 \%$ and a mitochondrial protein concentration of 2 $\mu \mathrm{g} / \mu \mathrm{l}$. For Tom 22 immunoprecipitations, mitochondria were resuspended in Tom 22 IP solubilization buffer at $2 \mu \mathrm{g} / \mu \mathrm{l}$, with or without previous subjection to in organello photo- 
crosslinking as explained in section 2.7.4. After solubilization, the clarified supernatant was applied to the appropriate resin and subjected to mild agitation for $1-2 \mathrm{hr}$ at $4{ }^{\circ} \mathrm{C}$. Following binding, the unbound fraction was removed and washing with digitonin wash buffer was carried out 10 times with ample washing buffer. Bound proteins were eluted from the columns with glycine elution buffer twice with three column volumes of buffer at room temperature for $5 \mathrm{~min}$ under gentle agitation. Bound proteins in HA immunoprecipitations were eluted with two column volumes HA peptide $(0.5 \mathrm{mg} / \mathrm{ml})$ in TBST at $4^{\circ} \mathrm{C}$ for $10 \mathrm{~min}$ under mild agitation. $1 \mathrm{M}$ Tris/ $\mathrm{HCl} \mathrm{pH} 8.0$ was added to all elutes to a final concentration of $500 \mathrm{mM}$, at which point proteins were precipitated with $20 \%$ tricarboxylic acid (TCA) and $0.0125 \%$ sodium deoxycholate for $15 \mathrm{~min}$ on ice. Insoluble precipitate was removed via centrifugation at $20,000 \mathrm{xg}$ for $10 \mathrm{~min}$ at $4{ }^{\circ} \mathrm{C}$. The supernatant was removed and the pellet resuspended in protein loading buffer and incubated at $95^{\circ} \mathrm{C}$ for $10 \mathrm{~min}$ and analyzed via SDS-PAGE and Western-blotting (see sections 2.6.1. and 2.6.5., respectively).

Following the elution of bound proteins, $\operatorname{IgG}$ bound resins were regenerated via washing with 10 column volumes of glycine elution buffer for $10 \mathrm{~min}$ at room temperature under mild agitation. The columns were neutralized with $100 \mathrm{mM}$ Tris/ $\mathrm{HCl} \mathrm{pH} \mathrm{8.0,} \mathrm{placed}$ in TBS buffer supplemented with $8 \mathrm{mM} \mathrm{NaN}_{3}$ and stored at $4{ }^{\circ} \mathrm{C}$ for future use.

\subsubsection{Metal affinity chromatography of the TOM complex}

Small-scale isolation of the TOM complex was performed utilizing mitochondria isolated from a yeast strain containing $\mathrm{a} \mathrm{His}^{10}$ at the C-terminus (Meisinger et al., 2001; Model et al., 2008). Mitochondria were solubilized in Tom $22^{\text {His }}$ solubilization buffer (small scale) at $1 \mu \mathrm{g} / \mu \mathrm{l}$ for 20 on ice, similar to section 2.7.3. Following incubation, clarification of the supernatant was performed via centrifugation at $20,000 \mathrm{xg}$ for $10 \mathrm{~min}$ at $4^{\circ} \mathrm{C}$. The supernatant was applied to Ni-NTA agarose $(25 \mu \mathrm{g}$ mitochondrial protein fraction/ $\mu \mathrm{l}$ resin). The mitochondrial lysate was let incubate with Ni-NTA for $1-2 \mathrm{hr}$ under gentle agitation at $4^{\circ} \mathrm{C}$. Unbound proteins were removed via low speed centrifugation $(100 \mathrm{xg})$ and the columns were washed ten times with Tom $22^{\text {His }}$ solubilization buffer (small scale) containing 0.3\% digitonin and $80 \mathrm{mM}$ imidazole. Bound proteins were eluted with Tom $22^{\text {His }}$ solubilization buffer (small scale) containing $0.3 \%$ digitonin and $200 \mathrm{mM}$ imidazole. Before photo-crosslinking of the eluate with $2 \mu \mathrm{M}$ photo-peptide, the buffer was exchanged to import buffer with $0.2 \%$ digitonin and without 
BSA through the use of a centrifugal filter. Photo-crosslinking was performed as in section 2.7.4., however, the washing step following UV irradiation was omitted and the samples were analyzed via SDS-PAGE and Western-blotting (see sections 2.6.1. and 2.6.5., respectively).

Large-scale isolation of the TOM complex was performed, in part, according to the previously published procedure (Ahting et al., 1999). Tom $22^{\text {His }}$ mitochondria were solubilized in Tom $22^{\text {His }}$ solubilization buffer (large scale) at $1 \mu \mathrm{g} / \mathrm{ml}$, and processed similarly to the small-scale purification protocol. After binding was performed as in the small-scale experiment, the column was washed with Tom $22^{\text {His }}$ solubilization buffer (large scale) containing $0.5 \%$ digitonin and $80 \mathrm{mM}$ imidazole ten times with ample buffer. Elution was performed with five column volumes of washing buffer containing $500 \mathrm{mM}$ imidazole and elutes were concentrated in a centrifugal filter. Following concentration, the buffer was exchanged to import buffer containing $0.5 \%$ digitonin without BSA via a PD G10 desalting column in accordance with the manufacture's instructions. Following centrifugal clarification of the purified, buffer exchanged TOM complex, photocrosslinking was performed identically to the small-scale experiment, and samples were analyzed via SDS-PAGE, colloidal Coomassie staining and nano LC-MS/MS (see sections 2.6.1., 2.6.4. and 2.6.7. for details, respectively).

\subsection{In vitro recombinant mitochondrial protein interaction assay}

$\operatorname{Tim} 21^{\mathrm{IMS}}$ purified from $E$. coli (see section 2.5.3. for details) and immobilized on CNBr-activated sepharose according to the manufacture's instructions in CNBr Coupling buffer overnight at $4^{\circ} \mathrm{C}$. Following coupling, the resin was quenched with $100 \mathrm{mM}$ Tris/HCl $\mathrm{pH} 8.0$ for $2 \mathrm{hr}$ at room temperature, washed with TBS buffer and then stored in TBS with $8 \mathrm{mM} \mathrm{NaN}_{3} .20 \mu \mathrm{l}$ of Tim $21^{\mathrm{IMS}}$ bound resin was mixed with $50 \mathrm{nM}$ Tim50 $0^{\mathrm{IMS}}$,

and various concentrations of $\operatorname{Tim} 23^{\mathrm{IMS}}$ or Tim $23^{\mathrm{YL} 70 \mathrm{AA}}$ in in vitro pull-down binding buffer. Following a $1 \mathrm{hr}$ binding incubation at $4^{\circ} \mathrm{C}$, the resin was washed with in vitro pulldown binding buffer ten times and eluted with three column volumes of glycine elution buffer. The eluate was then subjected to TCA precipitation as described in section 2.7.5., and analyzed via SDS-PAGE and Western-blotting for Tim50 (see sections 2.6.1. and 2.6.5. for details). 


\subsection{Homology modeling}

Homology modeling was performed essentially as previously published (Qiu et al., 2013). The S. cerevisiae Tom40 sequence was submitted to the HHpred server, which identified mouse VDAC1 (PDB ID 3EMN) (Ujwal et al., 2008) as the most promising homology modeling template as $15 \%$ sequence identity was found. The $S$. cerevisiae Tom40 homology model was generated through the use of Modeler (Soding et al., 2005), and images presented in this work were generated using Pymol. For aesthetic reasons, residues $1-48$ and $363-387$ were removed. 


\section{Results}

\subsection{Tom40 is a major contributor to TOM presequence interaction.}

With the aim of investigating presequence-interacting subunits of the TOM complex, previously established presequence probes were employed (Schulz et al., 2011). These presequence probes, schematically depicted in Fig. 8, were created using the sole structural presequence-receptor interaction data available (Abe et al., 2000), consisting of an NMR structure of rat Tom20 in complex with the presequence of rat aldehyde dehydrogenase (pALDH). Schulz and colleagues placed a UV-activatable crosslinking residue, para-benzoylphenylalanine (BPA), at defined locations within the pALDH, enabling the investigation of the hydrophobic and hydrophilic interaction surfaces with $\mathrm{pL}_{19} \mathrm{~B}$ and $\mathrm{pS}_{16} \mathrm{~B}$, respectively. Specifically, the BPA residues were positioned to reside on either side of the amphipathic $\alpha$-helix. Furthermore, with downstream mass spectrometry based analysis in mind, the authors placed an additional trypsin cleavage site within the photo-probes. Finally, both $\mathrm{pL}_{19} \mathrm{~B}$ and $\mathrm{pS}_{16} \mathrm{~B}$ were designed to contain a C-terminal biotinylated lysine and hexahistidyl tag for detection and photo-adduct purification purposes.

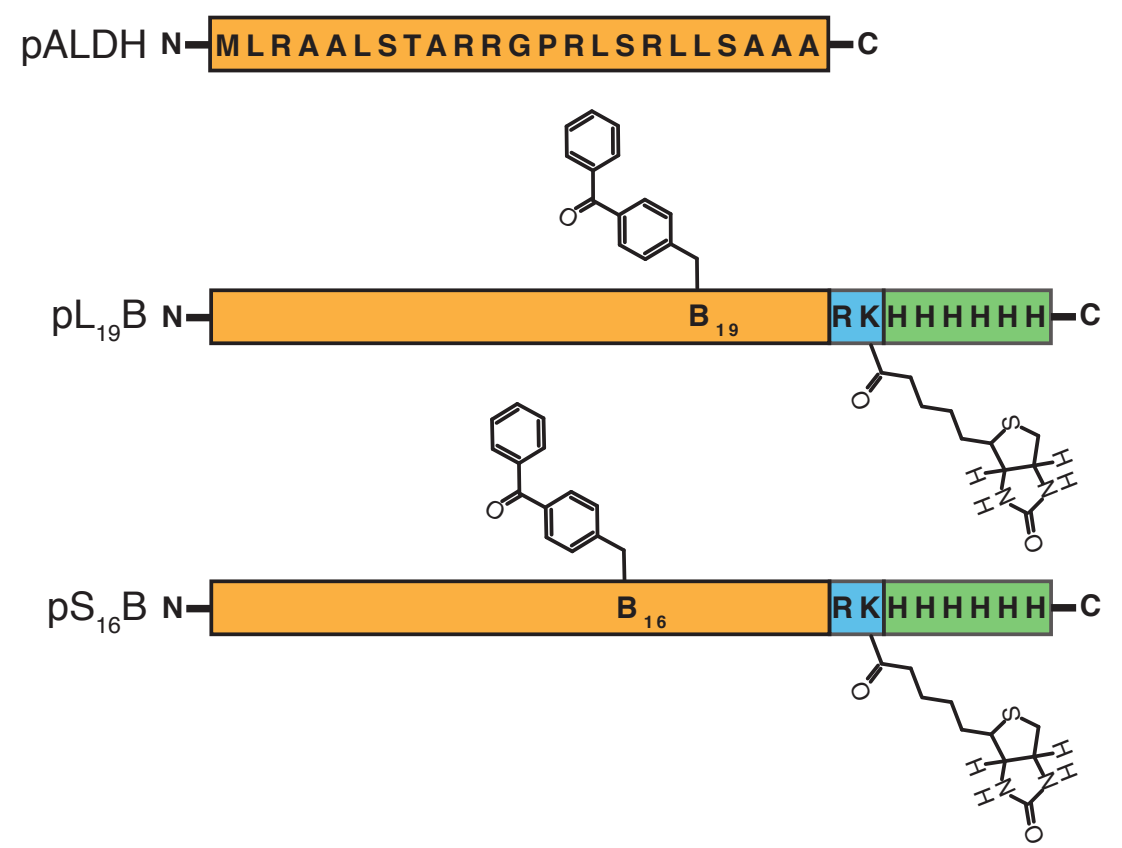

Figure 8. A schematic representation of the presequence probes. Presequence probes were created based upon the rat aldehyde dehydrogenase presequence (pALDH). The presequence probes contain the UV- activatable crosslinking residue para-benzoylphenylalanine (BPA) at key positions, leucine 
$19\left(\mathrm{pL}_{19} \mathrm{~B}\right)$ and serine $16\left(\mathrm{pS}_{16} \mathrm{~B}\right)$. Moreover, the presequence probes were also given an extra trypsin cleavage site and both a biotinylated lysine and hexahistidyl tag for detection and purification purposes, respectively.

Examining the presequence-interacting subunits of the TOM complex, the aforementioned presequence probes were used in conjunction with isolated wild-type mitochondria (Fig. 9). Upon separation of the in organello photo-crosslinking reaction via SDS-PAGE and detection of TOM subunits via Western-blotting, a photo-adduct could be seen with the core subunits of the TOM complex (Tom40 and Tom22), the TOM presequence receptors (Tom20 and Tom5) as well as the well established presequence receptor, Tim50 (Marom et al., 2011; Schulz et al., 2011) (Fig. 9, lanes 1 - 3). Importantly, the presequence probe negative controls, Fig. 9 lanes $4-6$, were shown not to exhibit presequence-binding activity.
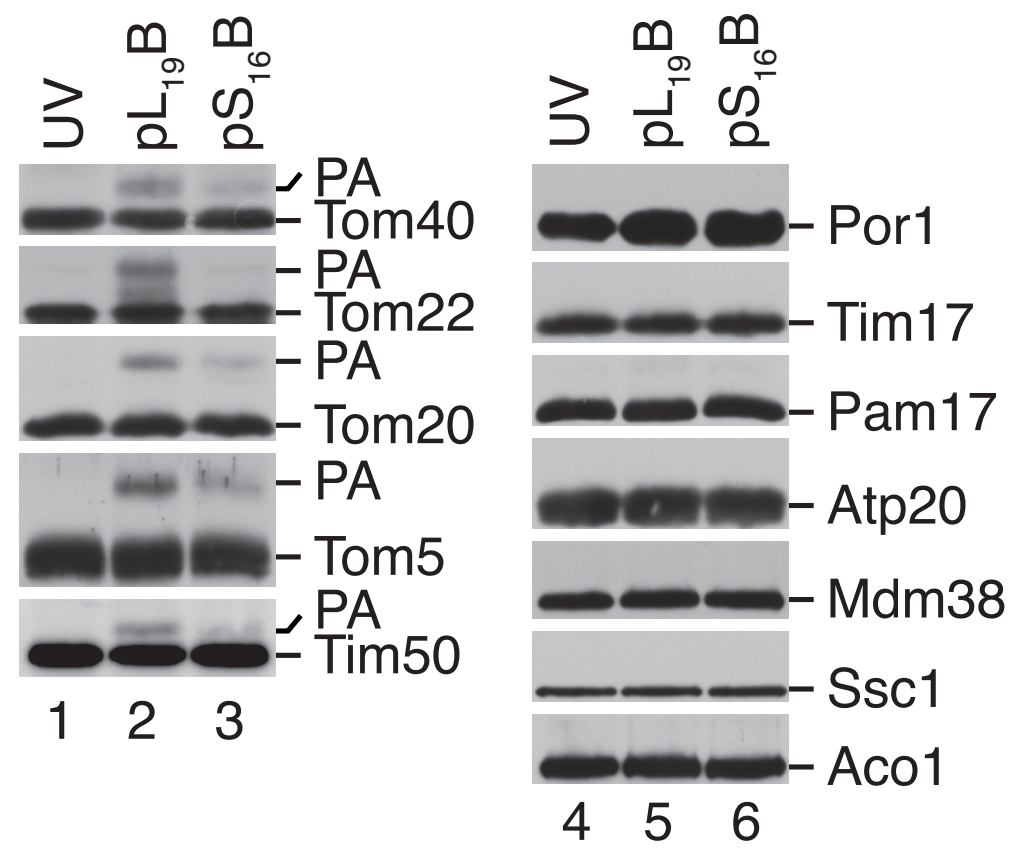

Figure 9. In organello photo-crosslinking in isolated wild-type mitochondria, detected via Western-blotting and probing for various proteins with specific antisera. PA, photo-adduct. Image kindly provided by Dr. Christian Schulz.

After establishing that TOM complex components form photo-adducts with presequence probes, the focus was shifted to Tom40, as a multitude of publications have alluded to possible functional interaction of Tom 40 with presequence-containing peptides in transit across the OM (Baker et al., 1990; Gaikwad and Cumsky, 1994; Gordon et al., 2001; Kanamori et al., 1999; Rapaport et al., 1997; 1998a; Stan et al., 2000; Vestweber et 
al., 1989). With efforts of determining Tom40's relative contribution of presequence interaction within the TOM complex, the translocase was immunoisolated from digitoninsolubilized isolated wild-type mitochondrial via Tom22 antiserum, having been previously subjected to in organello photo-crosslinking. Observed in Fig. 10A, the TOM complex was successfully isolated form the mitochondrial lysate as a clear enrichment of TOM subunits is seen (lanes $4-6$, as compared to lanes $1-3$ ), while the elates are devoid of other membrane integrated proteins of the OM (Por1) and matrix proteins (Aco1). Moreover, as seen in lanes $4-6$, the elution fractions contained photo-adducts of all known direct presequence interactors of the TOM complex, as well as Tom40. In order to assess the relative presequence probe photo-adduct formation by Tom 40 , as compared to other established TOM presequence receptors, the photo-adducts alone were detected via the integrated biotin tag through the use of SA-HRP. When the photo-adducts are detected alone in a uniform manner (Fig. 10B), Tom40 exhibits significant presequence probe photo-adduct formation, and therefore strongly suggests it plays an active role in the shuttling of presequence-containing substrates over the OM.

A

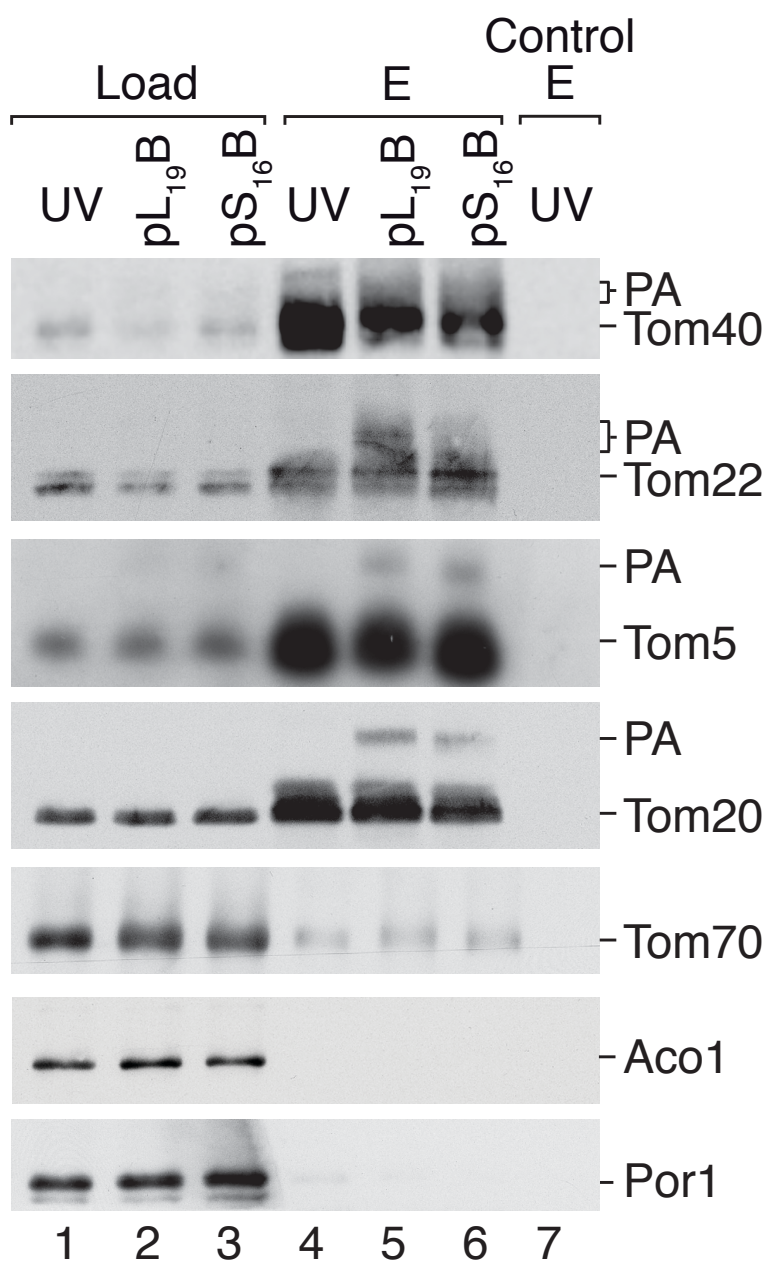

B
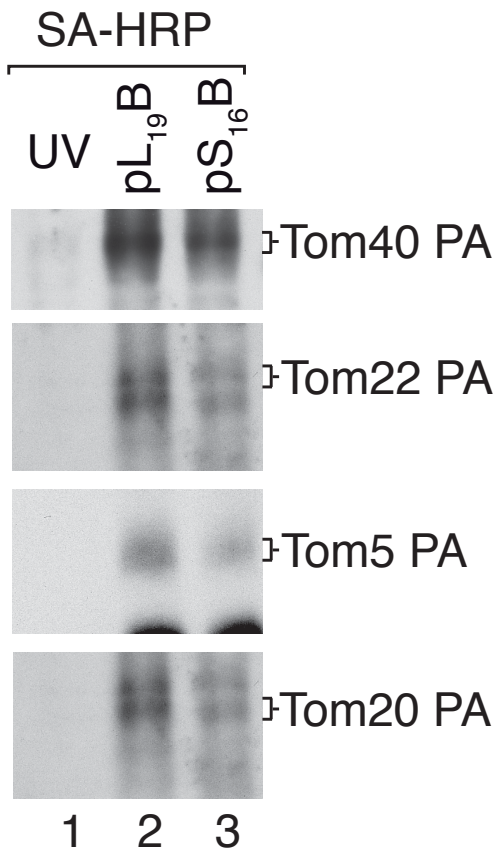
Figure 10. Immunoisolation of the TOM complex via Tom22 antiserum from digitoninsolubilized wild-type mitochondria, previously subjected to in organello photo-crosslinking. (A) Load and eluate (E) samples were resolved via SDS-PAGE and detected via Western-blotting and probing for various proteins with specific antisera. Control elution (lane 7) is from resin coupled to a nonrelated antibody. Load corresponds to $7 \%$ of the eluate. (B) Elution fractions from A were detected with SA-HRP. Load from A corresponds to $4 \%$ of the eluates in B. PA, photo-adduct. SAHRP, Streptavidin horseradish peroxidase.

Having established Tom 40 as a major contributor to presequence interaction at the TOM complex, the effect on the deletion of known presequence-binding subunits or domains flanking the Tom40 channel was assessed via presequence probe photocrosslinking. This analysis allowed for the investigation of the possible indirect Tom40 photo-adduct formation, as BPA has previously demonstrated a very dynamic active radius (3 - $15 \AA$ ) (Dormán and Prestwich, 1994; Schulz et al., 2011; Wittelsberger et al., 2006). As seen in Fig. 11, the Tom40 photo-adduct in mitochondria lacking Tom5 or the IMS domain of Tom22 was effectively unaltered, similarly to the established OM and IM presequence receptors, Tom 20 and Tim50, respectively.

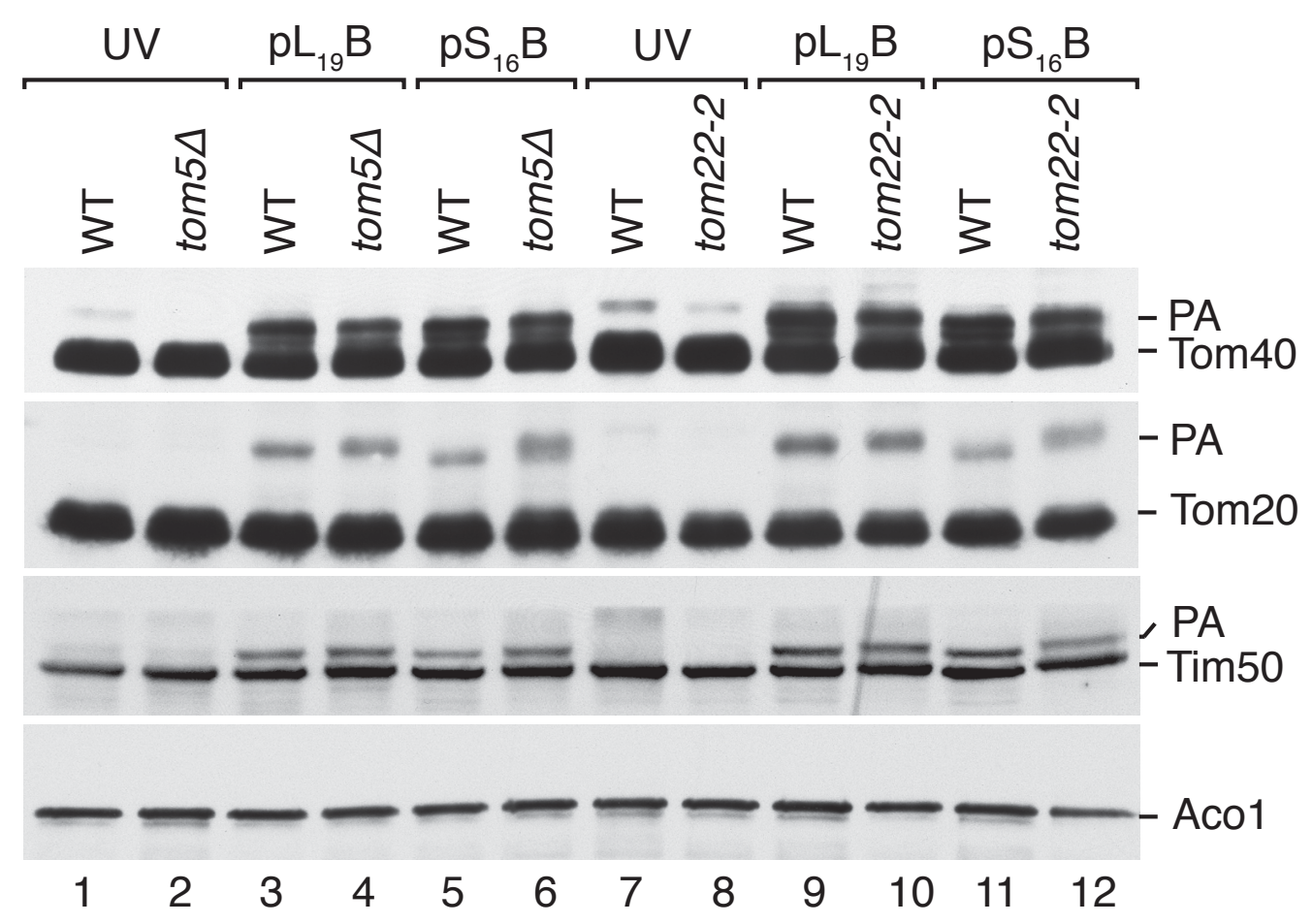

Figure 11. In organello photo-crosslinking in mutant and corresponding wild-type mitochondria. Samples were resolved via SDS-PAGE and detected via Western-blotting and probing for various proteins with specific antisera. PA, photo-adduct. 
The data set described above presents Tom40 as an independent presequence interactor of the TOM complex, which plays a significant presequence-binding role at the TOM complex.

\subsection{Tom40 displays two discrete presequence-interacting regions.}

During the creation of the presequence probes, the BPA residue was chosen specifically due to its very stable nature and, most importantly, upon UV irradiation stable C-C bonds are created (Dormán and Prestwich, 1994). As the result of the UV induced stable C-C covalent bond formation of the BPA photo-crosslink, downstream mass spectrometric pinpointing of the crosslink site was made possible. Therefore, the presequence-interacting regions of Tom40 were mapped through a combine photocrosslinking/mass spectrometry approach (Fig. 12). A prerequisite to mass spectrometry based ascertainment of the photo-crosslinking sites is the clean isolation of receptors yielding a significant subset of photo-crosslinked species in Coomassie stainable amounts. Therefore, a TOM isolation approach employing mitochondria containing a decahistidyl tag at the C-terminus of Tom22, in which the TOM complex was first isolated from digitonin-solubilized mitochondria and then subjected to photo-crosslinking was adopted, as portrayed in Fig. 12.

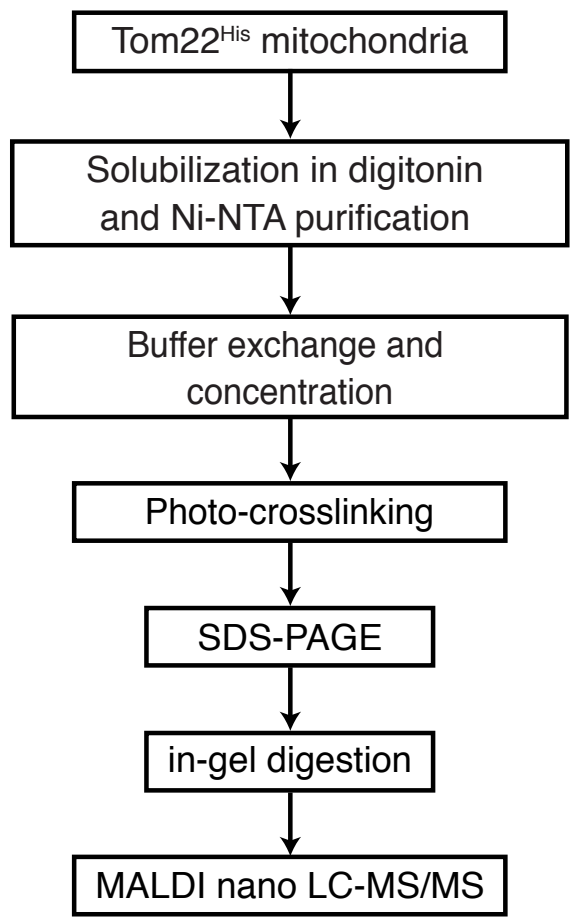

Figure 12. Workflow of joint photo-crosslinking/mass spectrometry approach to Tom40 presequence interaction mapping. 
Before preparative-scale TOM complex isolations were performed, small-scale photo-crosslinking was carried out with the Tom $22^{\mathrm{HIS}}$ Ni-NTA elutes. Observed in Fig. 13, the TOM complex is cleanly isolated from a Tom $22^{\mathrm{HIS}}$ mitochondrial lysate and TOM receptor photo-adducts are formed similarly to what was seen in in organello experiments. Importantly, a relatively high amount (approximately 50\%) of Tom40 is observed as a photo-adduct species.

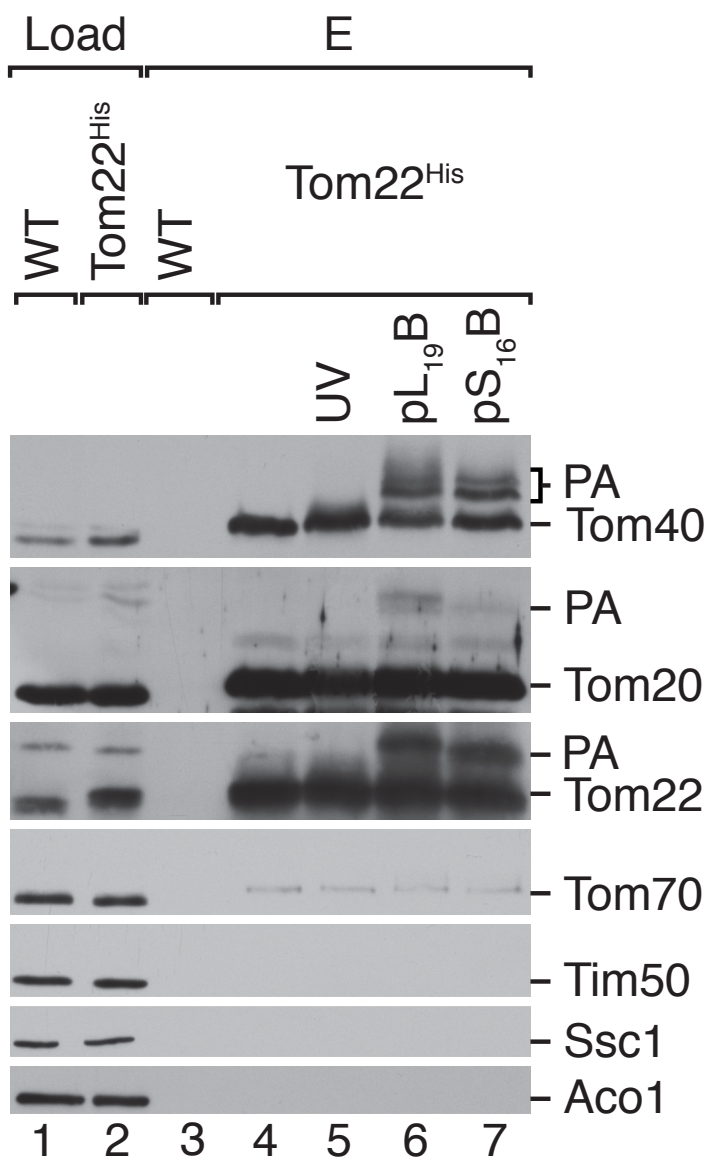

Figure 13. Ni-NTA isolation of the TOM complex from digitonin-solubilized Tom $22^{\mathrm{HIS}}$ mitochondria, followed by in vitro TOM complex photo-crosslinking. Samples were resolved via SDS-PAGE and detected via Western-blotting and probed for various proteins with specific antisera. PA, photo-adduct. Load 5\% of eluate (E). Image kindly provided by Dr. Christian Schulz.

Following the conformation of the small-scale test as seen in Fig. 13, preparativescale TOM complex isolations were performed, followed by photo-crosslinking as outlined in Fig. 12. As initial Ni-NTA mediated TOM isolation experiments only showed significant colloidal Coomassie staining for Tom40 (data not shown), an SDS-PAGE gel system optimized for the separation of Tom40 from its photo-adducts was chosen. Preparative-scale in vitro photo-crosslinking of Ni-NTA isolated yeast TOM complex led 
to the efficient ascertainment of colloidal Coomassie stainable photo-crosslinked Tom40 species (Fig. 14). It should be noted that approximately the same Tom40 photocrosslinking efficiency was achieved in both preparative-scale and small-scale experiments.

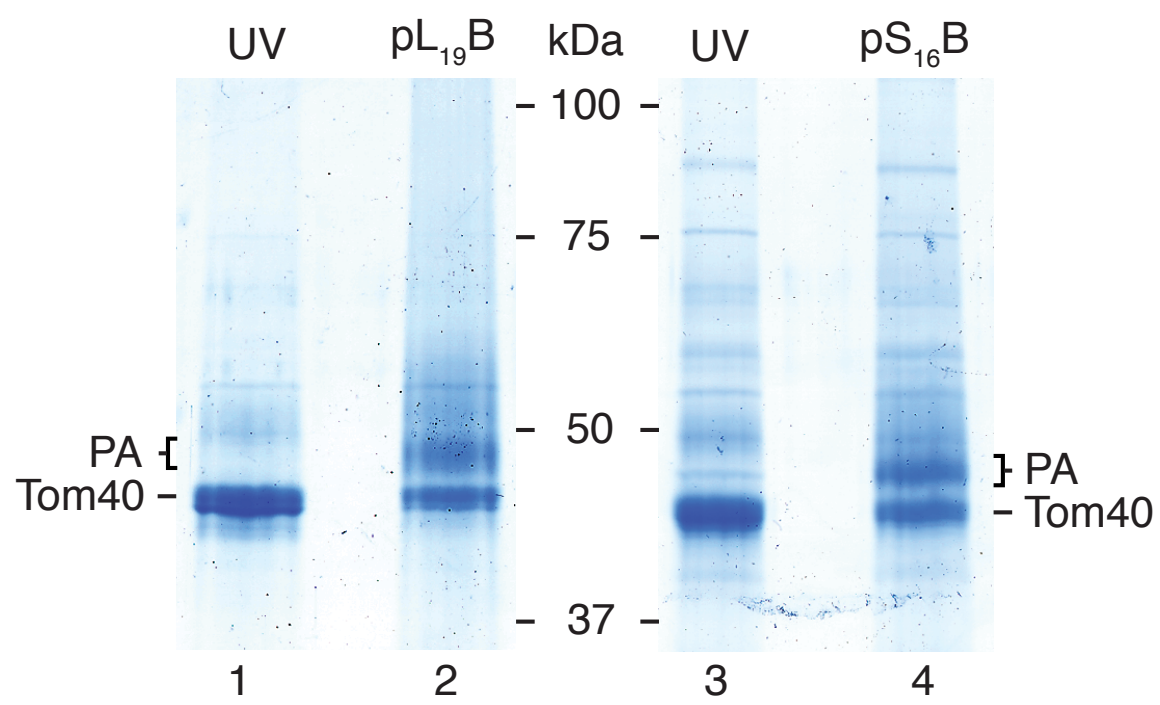

Figure 14. Preparative-scale Ni-NTA isolation of the TOM complex from digitonin-solubilized Tom $22^{\mathrm{HIS}}$ mitochondria, followed by in vitro TOM complex photo-crosslinking. Samples were resolved via SDS-PAGE and detected via colloidal Coomassie staining. PA, photo-adduct.

Colloidal Coomassie stained bands corresponding to Tom40 presequence probe photo-adducts were analyzed via MALDI nano LC-MS/MS. MS/MS spectra of tryptic fragments corresponding to photo-crosslink containing precursor masses are shown in Fig. 15 , with $\mathrm{A}-\mathrm{C}$ corresponding to $\mathrm{pL}_{19} \mathrm{~B}$ crosslinks and $\mathrm{D}$ and $\mathrm{E}$ corresponding to $\mathrm{pS}_{16} \mathrm{~B}$ crosslinks. The identification of a single crosslinked residue was, in one case, successful, with $\mathrm{M}^{94}$ of Tom40 identified to have been crosslinked to the BPA residue of $\mathrm{pL}_{19} \mathrm{~B}$. This identification was made possible due to the characteristic fragmentation pattern of the Met side chain when containing a BPA crosslink (Rodríguez-Castañeda et al., 2010; Schulz et al., 2011). However, other Tom40 tryptic fragments crosslinked to presequence probes could only be narrowed down to the dipeptide ${ }^{182} \mathrm{TL}^{183}$ (Fig. 15E) and the heptapeptide ${ }^{228}$ AGVSYLT $^{234}$ (Fig. 15A and D) due to the unforeseen instability of newly formed C-C bonds between BPA and Tom40 under the mass spectrometric sequencing conditions. 


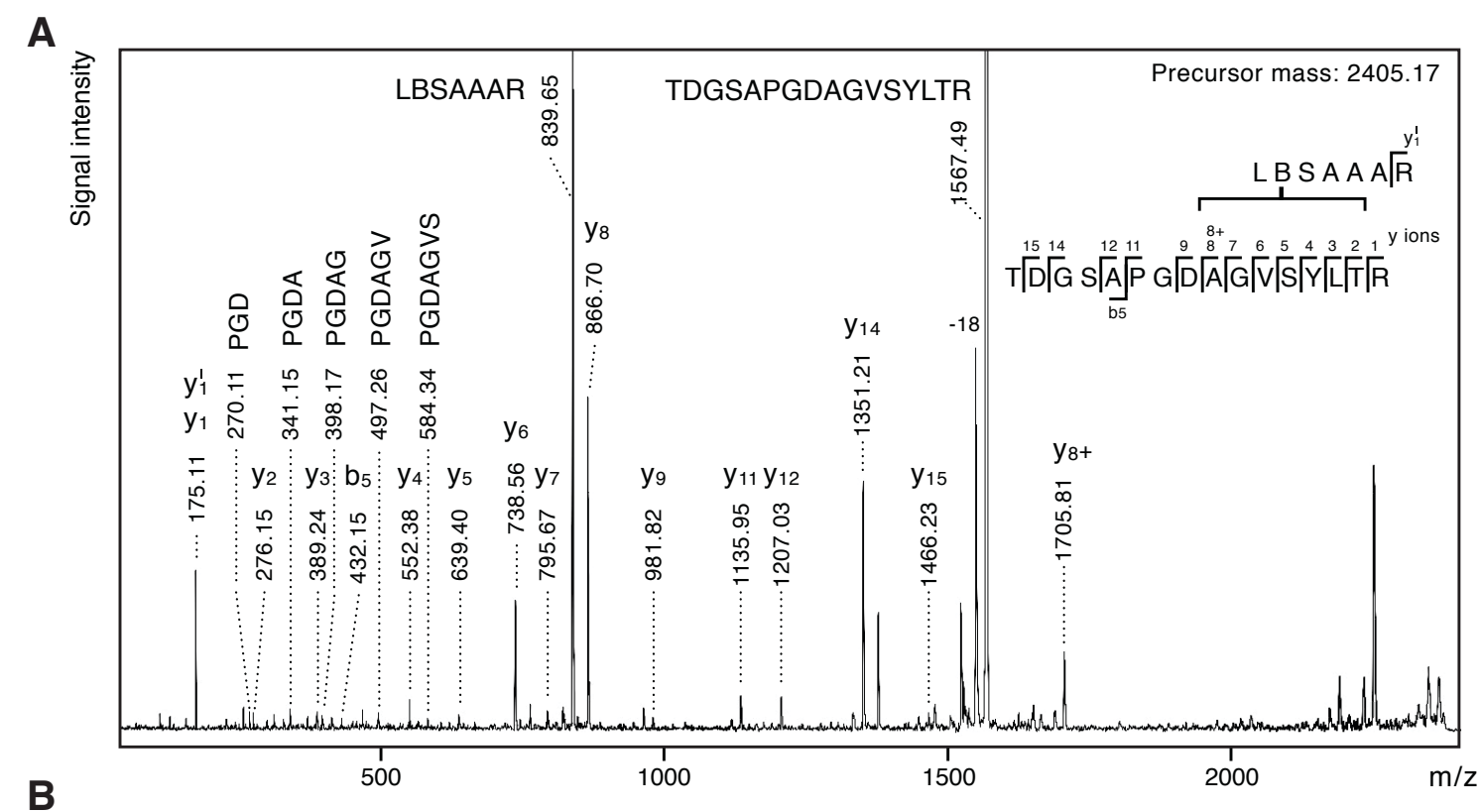

B

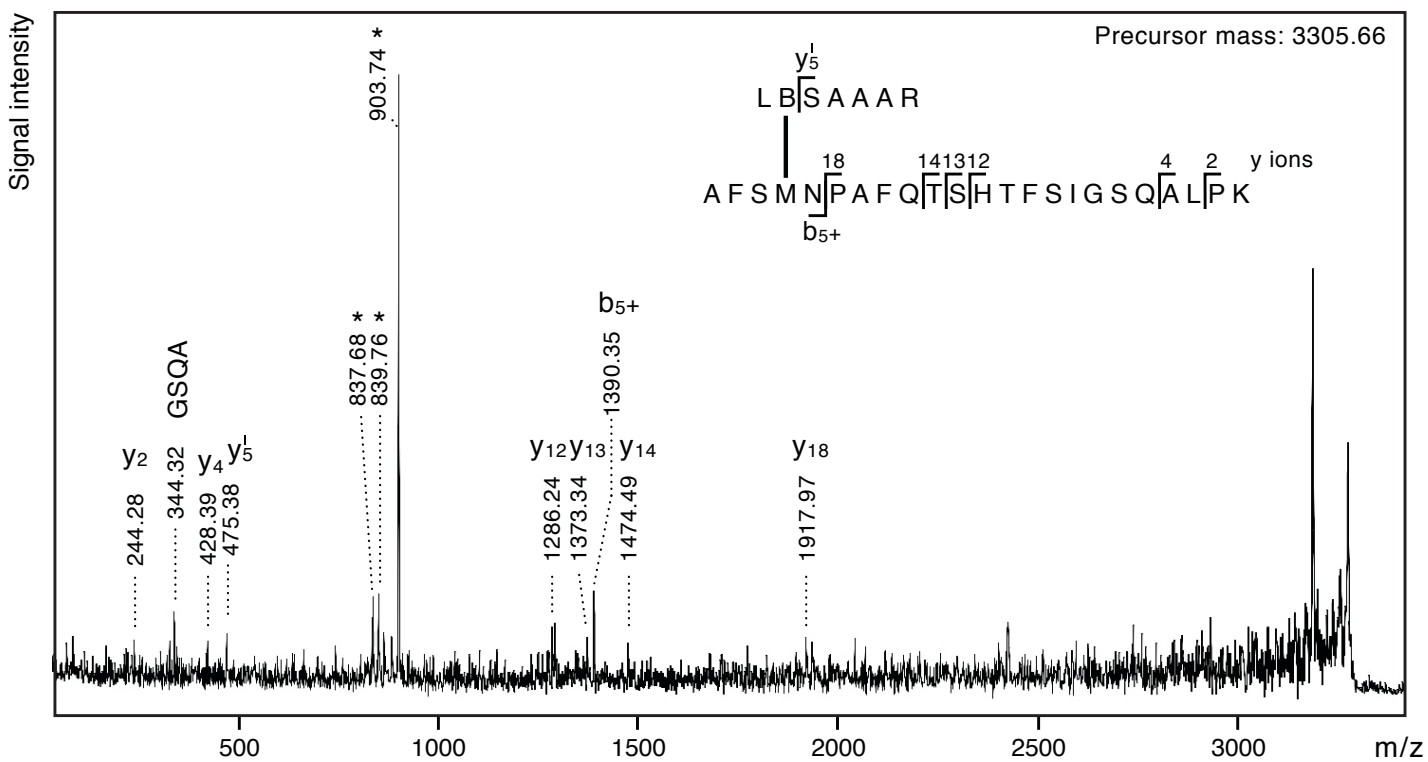

C

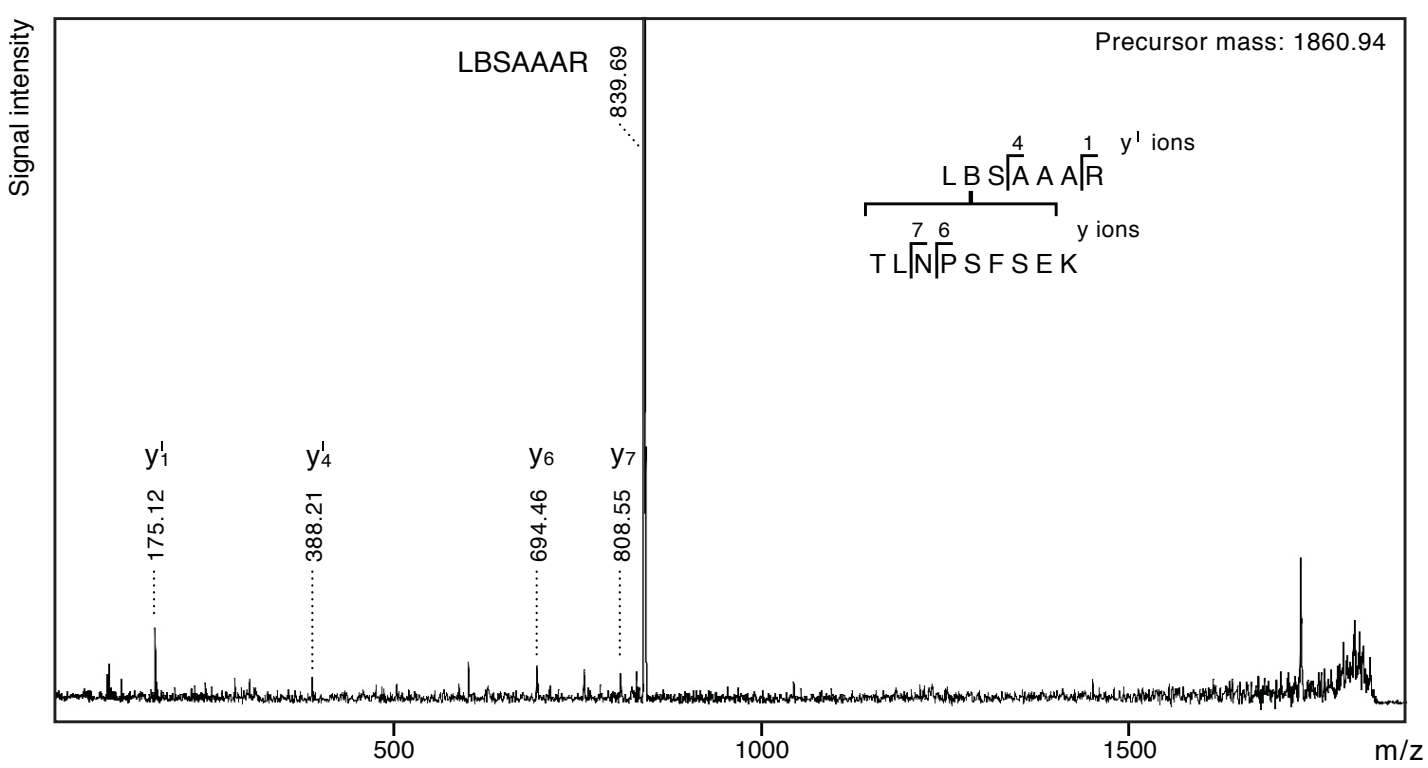



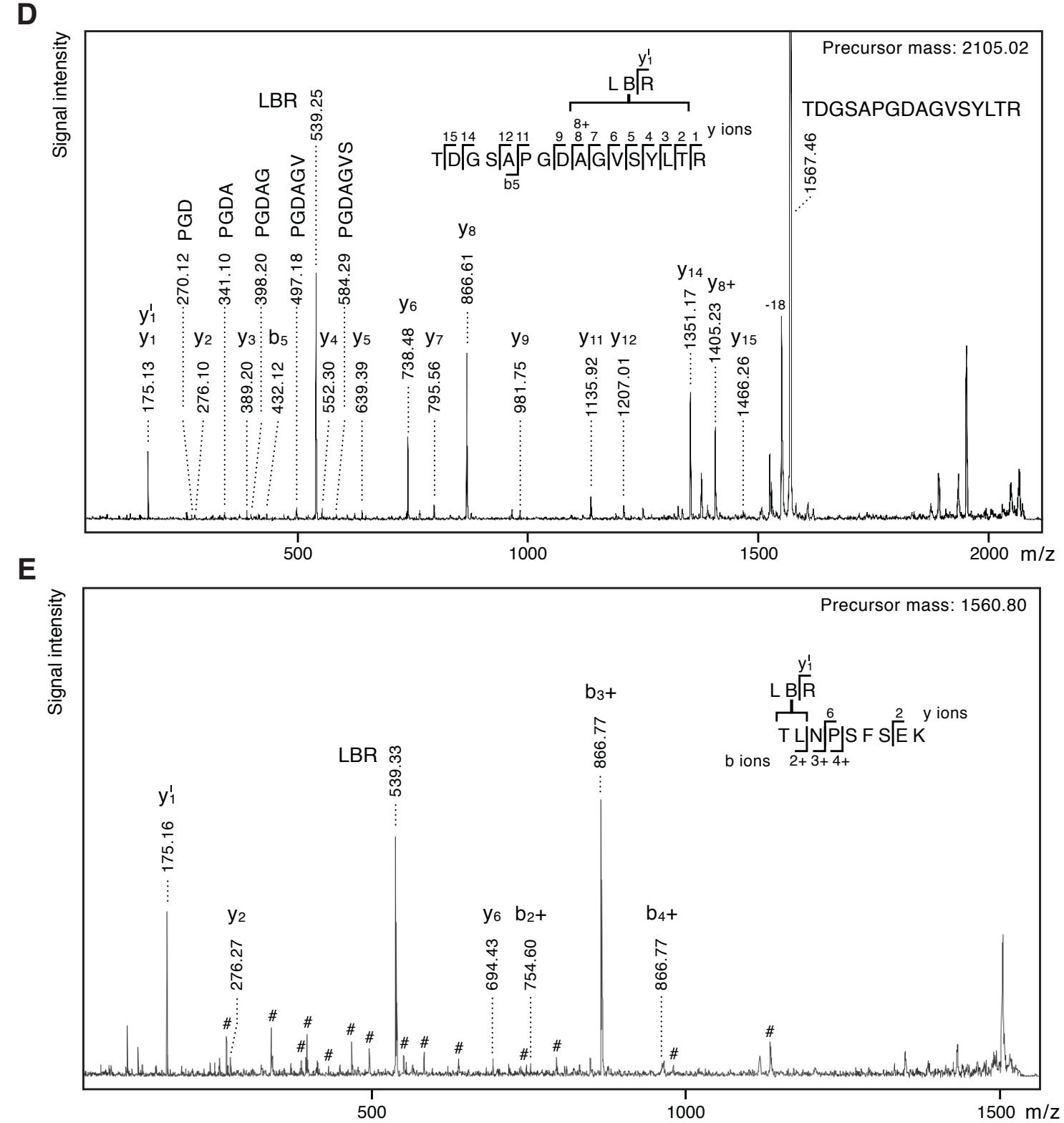

Figure 15. Fragment ion mass spectra of presequence-probe crosslinked Tom40 peptides. The band $y$-ions are indicated by $\mathrm{b}+$ and $\mathrm{y}+$ when carrying a crosslink to tryptic fragments of $\mathrm{pL}_{19} \mathrm{~B}(\mathrm{~A}-\mathrm{C})$ or $\mathrm{pS}_{16} \mathrm{~B}$ (D and $\left.\mathrm{E}\right)$. Fragment ions resulting from cleavage of the crosslink bond are labeled with the respective tryptic peptide sequence. (A) Tom $40^{220-235}$ crosslinked to $\mathrm{pL}_{19} \mathrm{~B}^{18-24}$; $[\mathrm{M}+\mathrm{H}]^{+}{ }_{\text {obs }}=2405.17$, $[\mathrm{M}+\mathrm{H}]_{\text {calc }}^{+}=2405.17$; internal fragments with $\mathrm{N}$-terminal Pro are indicated; the $\mathrm{y}_{8+}-$ ion identifies ${ }^{228}$ AGVSYLT $^{234}$ as the minimal crosslinking site. (B) Tom $40^{91-113}$ crosslinked to $\mathrm{pL}_{19} \mathrm{~B}^{18-24} ;[\mathrm{M}+\mathrm{H}]^{+}$obs $=3305.66,[\mathrm{M}+\mathrm{H}]^{+}$calc $=3305.64$. The $\mathrm{b}_{5+}$-ion together with the indicative triplet signal (marked by asterisks) identified $\mathrm{M}^{94}$ as the crosslinking site. (C) Tom $40^{182-190}$ crosslinked to $\mathrm{pL}_{19} \mathrm{~B}^{18-24} ;[\mathrm{M}+\mathrm{H}]^{+}$obs $=1860.94,[\mathrm{M}+\mathrm{H}]_{\text {calc }}^{+}=1860.95$. (D) Tom $40^{220-235}$ crosslinked to $\mathrm{pS}_{16} \mathrm{~B}^{15-17} ;[\mathrm{M}+\mathrm{H}]_{\text {obs }}^{+}=2105.02$, $[\mathrm{M}+\mathrm{H}]_{\text {calc }}^{+}=2105.03$; the $\mathrm{y}_{8+}$-ion identifies ${ }^{228} \mathrm{AGVSYLT}^{234}$ as the minimal crosslinking site. (E) Tom $40^{182-190}$ crosslinked to $\mathrm{pS}_{16} \mathrm{~B}^{15-17} ;[\mathrm{M}+\mathrm{H}]_{\text {obs }}^{+}=1560.80,[\mathrm{M}+\mathrm{H}]_{\text {calc }}^{+}=1560.81$; the $\mathrm{b}+$ ion series identifies ${ }^{182} \mathrm{TL}^{183}$ as the minimal crosslinking site; \# indicates signals corresponding to fragmentation 
of another nearly isobaric Tom40 peptide co-isolated in the precursor selection window. Measurements performed in close cooperation with Drs. Bernhardt Schmidt and Olaf Jahn.

Fortunately, the recent presentation of both a limited proteolysis and site-specific crosslinking verified Tom40 homology model allowed for the placement of mass spectrometry derived presequence-interacting regions (Gessmann et al., 2011; Qiu et al., 2013). When the three identified presequence interaction regions are placed within the homology structure (Fig. 16 A - C), two spatially distinct presequence active sites are seen, the first uncovered through the crosslink to $\mathrm{M}^{94}$ (Fig. 16, III) and the second highlighted by multiple crosslinking sites on the opposite end of the B-barrel (Fig. 16, I and II).

A

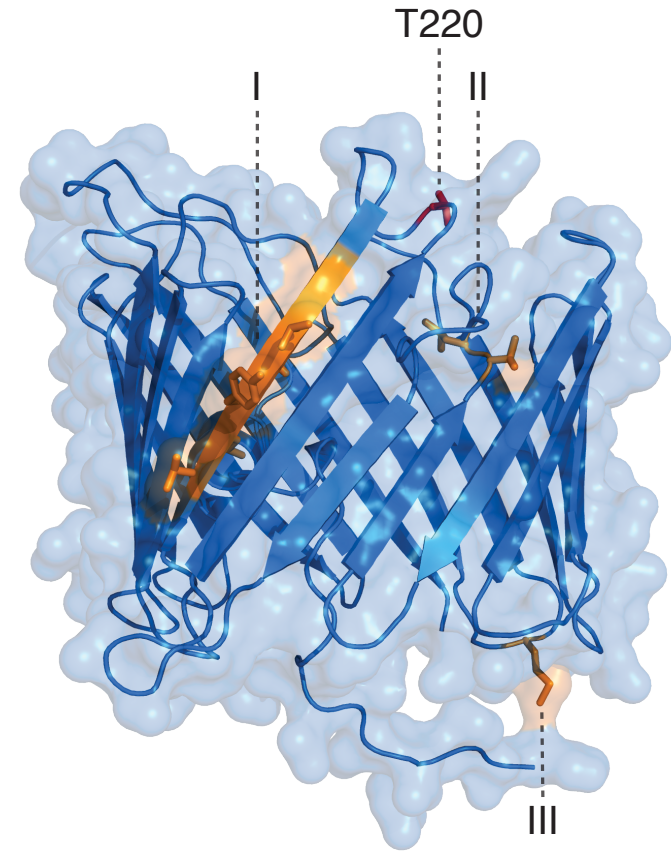

B

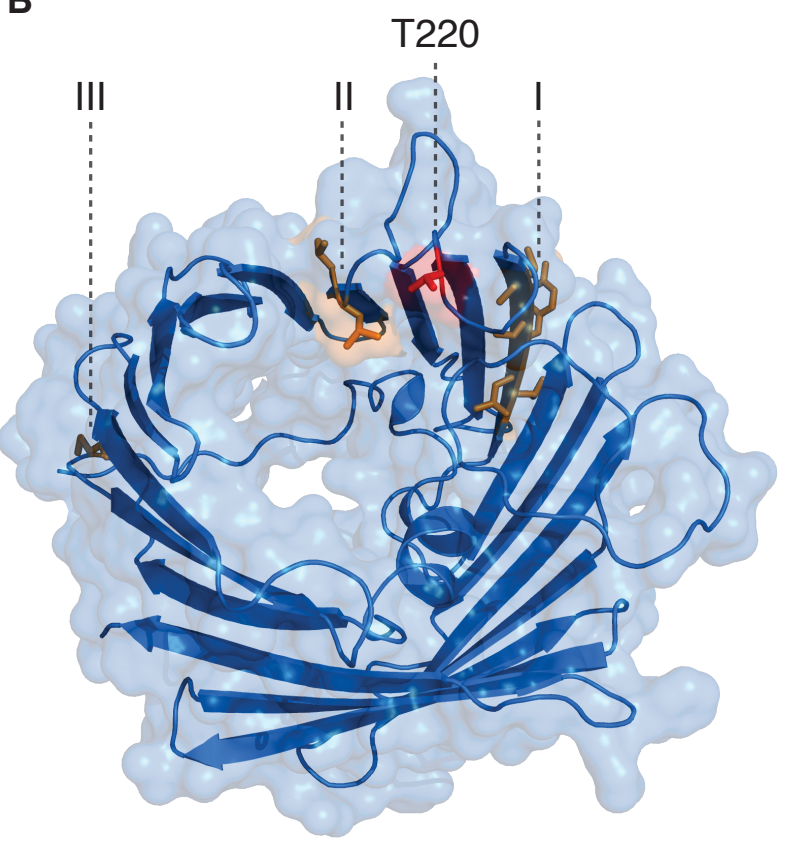

C

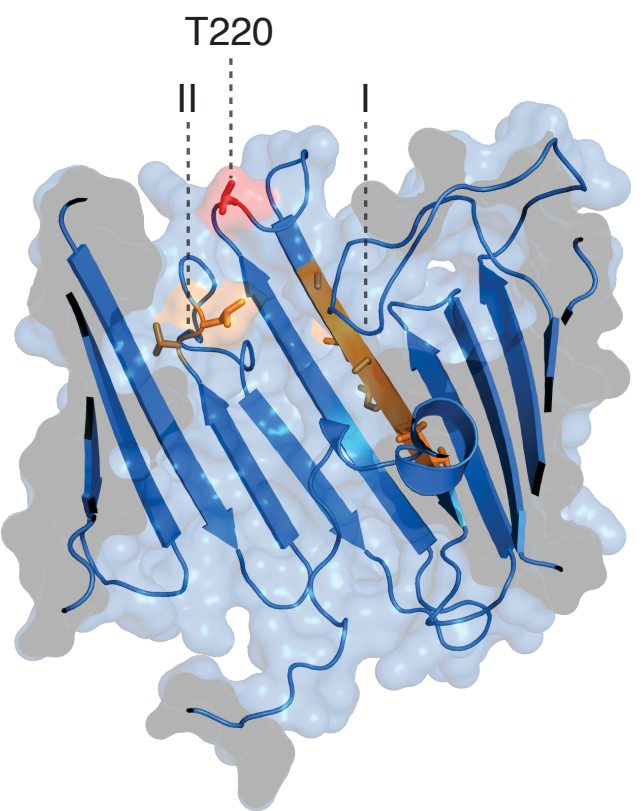


Figure 16. Tom40 homology model based upon the mouse VDAC structure (PDB ID 3EMN). Photo-crosslinked residues or fragments in orange and T220 in red. I, ${ }^{228}{ }^{A G V S Y L T}{ }^{234}$. II, ${ }^{182} \mathrm{TL}^{183}$. III, $\mathrm{M}^{94}$. (A) lateral view, (B) top view and (C) lateral cutout view.

Fortuitously, one of the two presequence-interacting sites of Tom40 was outlined to a higher resolution (Fig. 16, I and II), allowing for the more targeted analysis of key resides in the pocket, with the aim of presequence-specific Tom40 mutant construction.

\subsection{TOM complex stability is unaltered in Tom40 phosphomimetic (T220E) and phosphoblock (T220V) mutants.}

Examining a recent publication on the phospho-status of the TOM complex (Schmidt et al., 2011), the phosphorylation of Tom40 was seen to take place within the center of the identified presequence-interacting region by a presently elusive kinase. This residue, T220 (Fig. 16, highlighted in red), was then utilized in the conformation of the presequence-interacting region as well as the functional characterization of the binding site, through the creation of the phospho-mimetic mutant T220E and the phospho-block mutant T220V. Importantly, this allowed for physiologically grounded Tom40 mutant construction, thereby avoiding compounding effects from the well-known assembly and stability pitfalls of mutating Tom40 (Gabriel et al., 2003; Sherman et al., 2006).

Creation of the Tom40 mutants was accomplished through the transformation of a tom $40 \Delta$ yeast strain carrying a plasmid encoding TOM4O along with a URA3 selection marker (Kutik et al., 2008; Qiu et al., 2013). This strain was transformed with a pFL39 plasmid encoding tom $40^{E m u t}$ or tom $40^{V m u t}$, as well as the TRP1 selection marker. Following transformation, yeast were grown on synthetic solid media lacking both uracil and tryptophan, allowing for the retention of both TOM40 allele containing plasmids. Colonies were then subjected to 5-FOA mediated plasmid loss, triggering the growth of only cells which have lost the wild-type TOM40 allele containing plasmid as it also contained the URA3 selection marker. As seen in Fig. 17A, 5-FOA induced plasmid loss clearly indicates the viability of the newly created TOM4O alleles, as cells demonstrated wild-type-like colony formation. Moreover, the negative control strain, lacking a nonURA3 containing TOM40 allele plasmid, was, as expected, unable to form colonies on the 5-FOA containing media. Furthermore, an in-depth yeast dilution growth analysis (Fig. 
17B) illustrated completely unaltered growth on non-fermentable media across a wide range of temperatures.

A
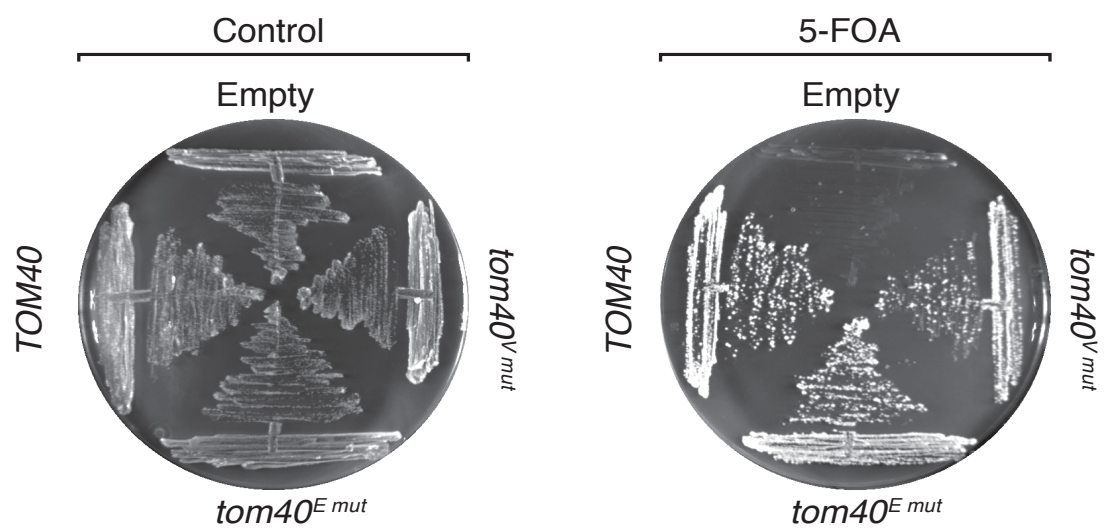

B

$24^{\circ} \mathrm{C}$

$30^{\circ} \mathrm{C}$

$37^{\circ} \mathrm{C}$
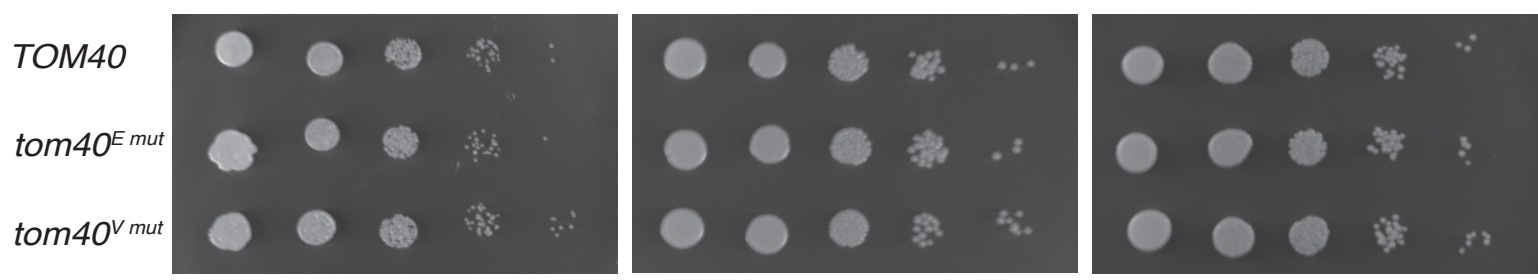

Figure 17. $\operatorname{tom} 40^{E m u t}$ and $\operatorname{tom} 40^{V m u t}$ alleles display no growth phenotype and wild-type-like TOM stability. (A) Yeast containing a chromosomal deletion of TOM40, complemented by wild-type TOM40 within a URA3 selection plasmid, were transformed with plasmids containing TOM40 alleles as indicated and subjected to plasmid loss on 5-FOA containing medium. (B) Serial dilutions (10fold) of the indicated strains were incubated at the indicated temperature for three days on nonfermentable medium.

Mitochondria were isolated from the yeast strains containing the phospho-mutant TOM40 alleles and selected mitochondrial proteins were examined through SDS-PAGE and Western-blotting utilizing specific antisera (Fig. 18A). As hinted upon by the dilution based growth test in Fig. 12B, no differences in mitochondrial protein levels were detected upon comparison of the mutant Tom40 containing mitochondrial (Fig. 18A, lanes 4-9), as compared with the corresponding wild-type (Fig. 18A, lanes $1-3$ ). Importantly, the Tom40 levels as well as the rest of the TOM complex subunits examined were seen to display unaltered protein amounts both among the mutants and compared to the wild-type.

The stability of the TOM complex was monitored through BN-PAGE separation of digitonin-solubilized mitochondria. As shown in Fig. 18B, TOM complex migration, when detected via Tom40, was unaltered across the phospho-mutants as specific signal was observed at equal heights within the native gel. Moreover, complexes III and IV in the 
Tom40 mutant containing mitochondria, detected via Rip1 and Atp5 respectively, showed unchanged migration on BN-PAGE. Taken together, the aforementioned protein level and stability assays demonstrate unaltered TOM characteristics and support the subsequent functional analysis of the Tom40 phospho-mutants, as non-specific protein level and stability issues are not present.
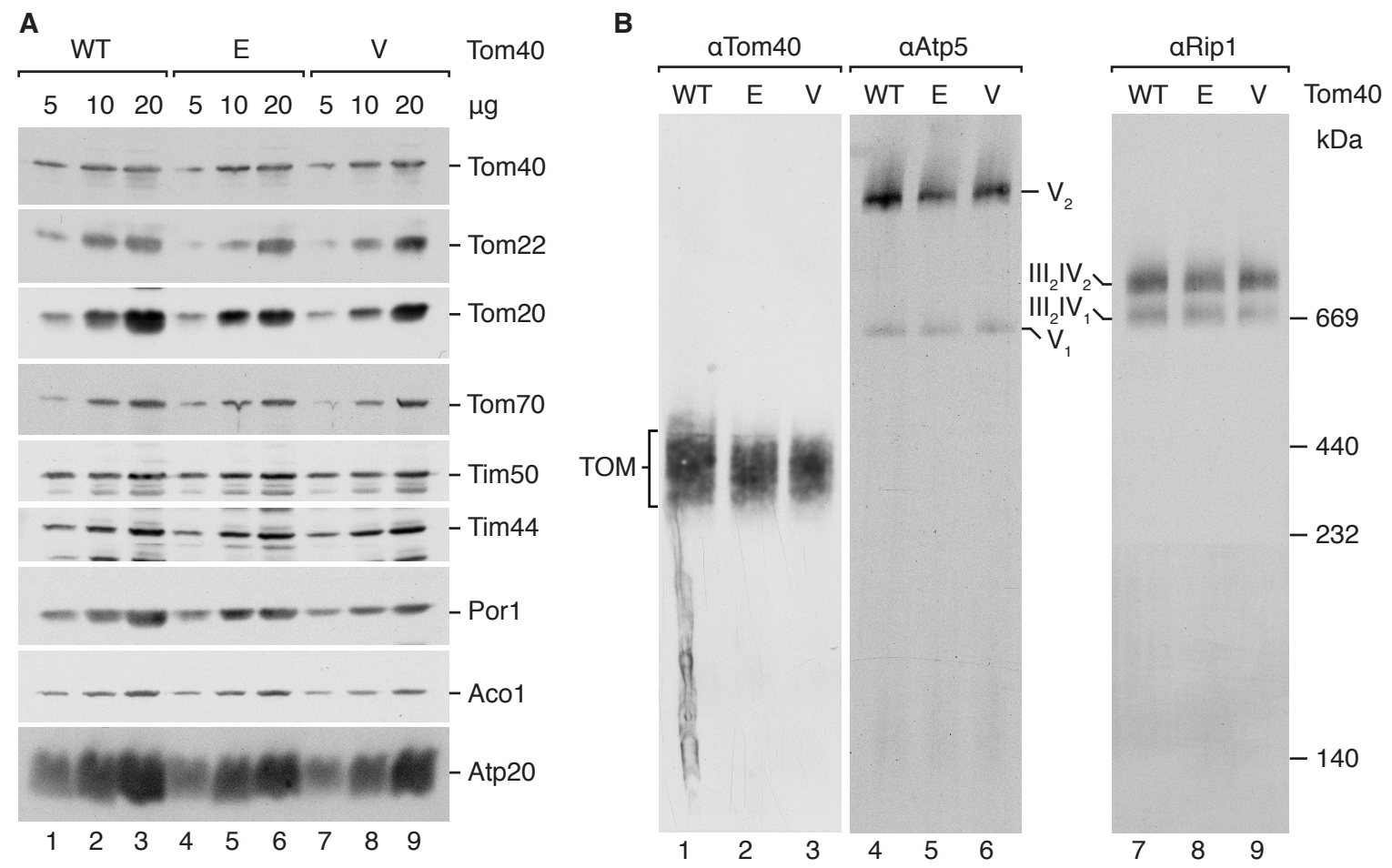

Figure 18. Tom40 phospho-mutants exhibit wild-type-like mitochondrial protein levels and TOM stability. (A) Western blot analysis of steady-state protein levels in indicated strains from isolated mitochondria via SDS-PAGE using specified antisera. (B) BN-PAGE Western blot analysis of steady-state complex levels using antisera for Tom40, Atp5 and Rip1 using $40 \mu \mathrm{g}, 15 \mu \mathrm{g}$ and $15 \mu \mathrm{g}$ isolated mitochondrial protein respectively.

\subsection{Import analysis of Tom40 phospho-mutants.}

Following the TOM protein steady-state levels and stability analysis, conformation of the presequence specificity of the mutants was carried out, through the use of photocrosslinking with the $\mathrm{pL}_{19} \mathrm{~B}$ (Fig. 19). In this in organello photo-crosslinking experiment, $\mathrm{pL}_{19} \mathrm{~B}$ was chosen as it was designed to monitor the hydrophobic presequence receptor interface, therefore its photo-adducts are expected to exhibit resilience in the higher salt conditions implemented in the experiment. As a specific Tom40 photo-adduct reduction was seen in the Tom $40^{\mathrm{E}}$ mutant in the in organello photo-crosslinking experiment, the 
presequence-binding significance of the newly mapped Tom40 binding site could be confirmed. Importantly, presequence probe photo-adduct formation with established IM presequence translocase receptors $\operatorname{Tim} 23$ and $\operatorname{Tim} 50$ was not reduced in $\operatorname{Tom} 40^{\mathrm{E}}$ mitochondria. It should be noted that the reduction in Tom40 photo-adduct formation in Tom $40^{\mathrm{E}}$ mitochondria couldn't be simply due to the unspecific introduction of a negatively charged residue in a key area. The presequence exhibits a net positive charge which, if only electrostatic interaction were at play, should increase the presequence-binding affinity, yet a decrease in Tom40's photo-adduct was seen in this strain. This notion also strongly suggests that a more complicated presequence-binding regulatory mechanism is at play within Tom40. This key finding allows for both the validation of the joint presequence probe photo-crosslinking/mass spectrometry approach, as well as demonstrating Tom40's direct role in presequence-binding.

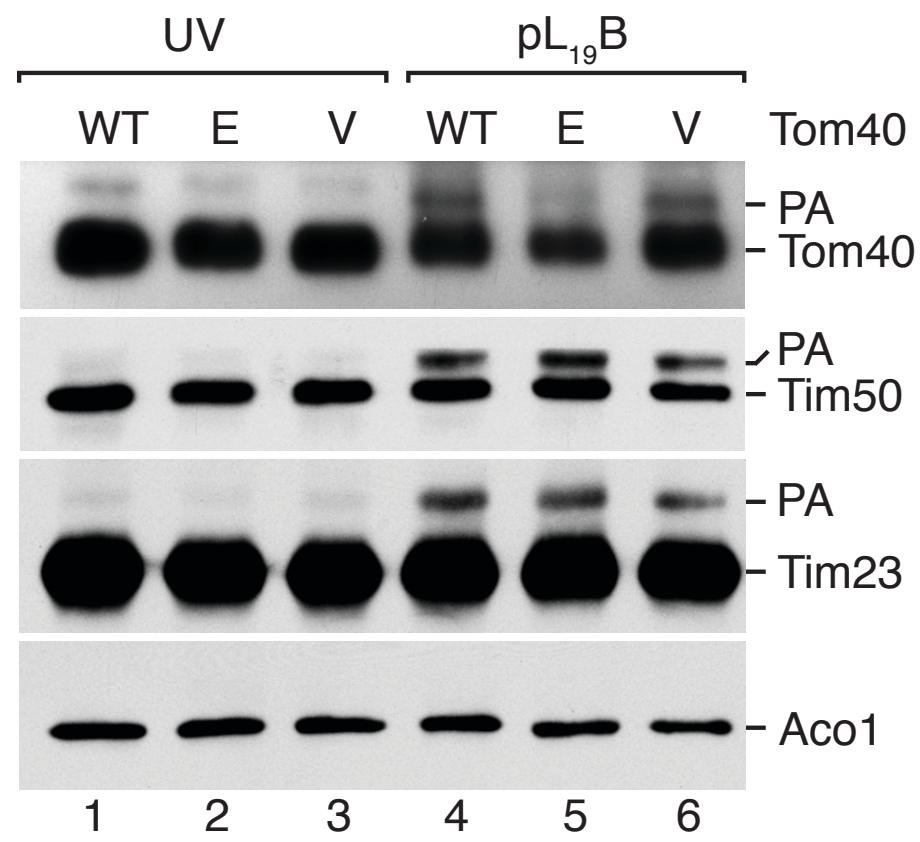

Figure 19. Photo-crosslinking in isolated $\operatorname{Tom}_{40} \mathrm{E}$ and $\mathrm{Tom}_{40}^{\mathrm{V}}$ mitochondria. (A) In organello photo-crosslinking analysis in isolated $\operatorname{Tom} 40^{\mathrm{E}}$ and $\operatorname{Tom} 40^{\mathrm{V}}$ mitochondria, imaged via Westernblotting utilizing the indicated antisera. PA, photo-adduct.

After conformation of presequence-binding augmentation within the Tom40 phospho-mutants, functional import analyses were performed, with the non-presequence related carrier and MIA pathways monitored. The carrier pathway was assessed through the import and assembly of $\left[{ }^{35} \mathrm{~S}\right] \mathrm{AAC}$, followed by the native separation of digitonintreated mitochondria lysate and digital autoradiography (Fig. 20A). Tom40 mutant 
mitochondria displayed an unaltered ability in the assembly of the carrier pathway substrate. Moreover, $\left[{ }^{35} \mathrm{~S}\right] \mathrm{Tim} 9$, a MIA pathway substrate, was urea denatured and imported into Tom40 mutant mitochondria, separated via reducing SDS-PAGE and analyzed via digital autoradiography (Fig. 20B). When evaluating the MIA pathway import capacity of the Tom40 phospho-mutants, no striking difference was found. It should be mentioned that a slight reduction in Tom $40^{\mathrm{V}}$-containing mitochondria was observed, yet the reduction was within the range of error of the corresponding wild-type.

A

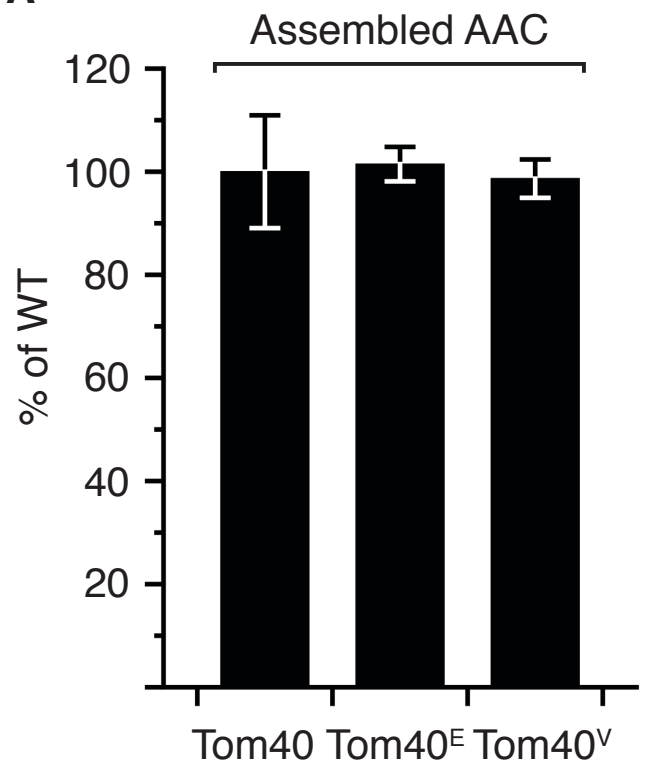

B

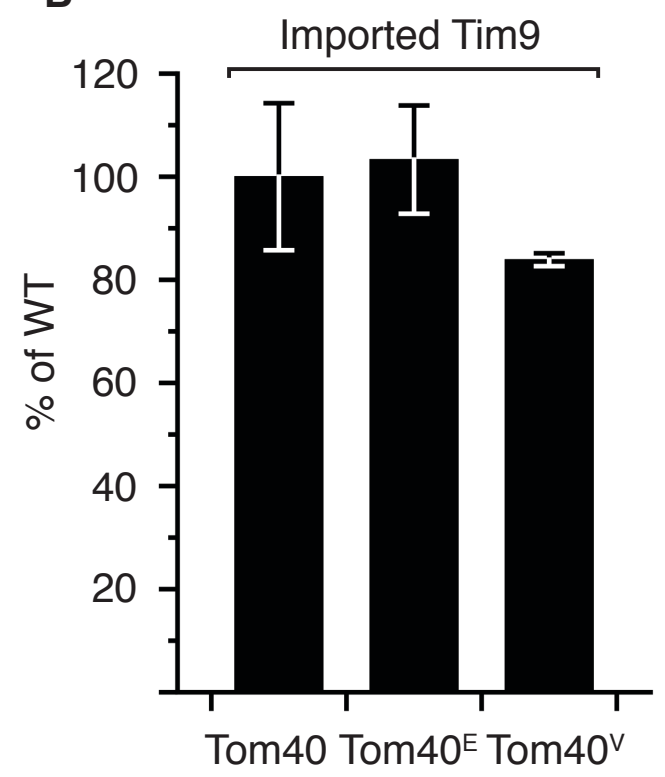

Figure 20. Carrier and MIA pathway import analyses in Tom40 phospho-mutant mitochondria. (A) $\left[{ }^{35} \mathrm{~S}\right] \mathrm{AAC}$ was imported and assembled into isolated mitochondria from the indicated strains, followed by digitonin solubilization and BN-PAGE. The fully assembled AAC dimer was quantified via digital autoradiography and presented as $\%$ of wild-type $(n=4$, SEM). (B) Urea denatured $\left[{ }^{35}\right.$ S]Tim9 was imported into isolated mitochondria and resolved via reducing SDS-PAGE. Proteinase K protected imported Tim9 was quantified and presented as \% wild-type ( $n=3$, SEM). Tim9 import experiments were performed by Dr. Lidia Wrobel (Warsaw, Poland).

\subsection{Functional characterization of TOM translocation intermediates within Tom40 phospho-mutants.}

Following the establishment of TOM functionality through the import of both carrier and MIA pathway substrates, the translocation of a presequence-containing precursor was monitored during TOM passage. In this assay, Oxa1, a presequencecontaining substrate targeted to the mitochondrial matrix via TOM and TIM23, was imported into Tom 40 phospho-mutant mitochondria under conditions in which the 
membrane potential was depleted through the addition of an inhibitor cocktail (AVO antimycin A, valinomycin and oligomycin) prior to commencing the import reaction. Under these conditions the Oxal substrate is known to produce a stable and productive TOM complex intermediate (Frazier et al., 2003). Therefore, $\left[{ }^{35} \mathrm{~S}\right] \mathrm{Oxa} 1$ was imported into Tom40 phospho-mutant mitochondria with and without $\Delta \psi$, solubilized in digitonin buffer, resolved via BN-PAGE and imaged through digital autoradiography (Fig. 21). Evaluating the Oxa1 TOM (known within this assay as the general import pore [GIP]) intermediate within the Tom40 phospho-mutants, a striking alteration in presequence mediated TOM translocation kinetics is seen. The Tom $40^{\mathrm{V}}$-containing mitochondria show an unexpected increase in TOM translocation kinetics when compared to both the wild-type and the Tom $40^{\mathrm{E}}$-containing mitochondria, resulting in a $43 \%$ increase in presequence translocation capacity at the $30 \mathrm{~min}$ time point. This finding thus allows for the Tom40-presequenceprobe mapped binding site investigated in this study to be functionally connected to presequence mediated import across the mitochondrial OM.

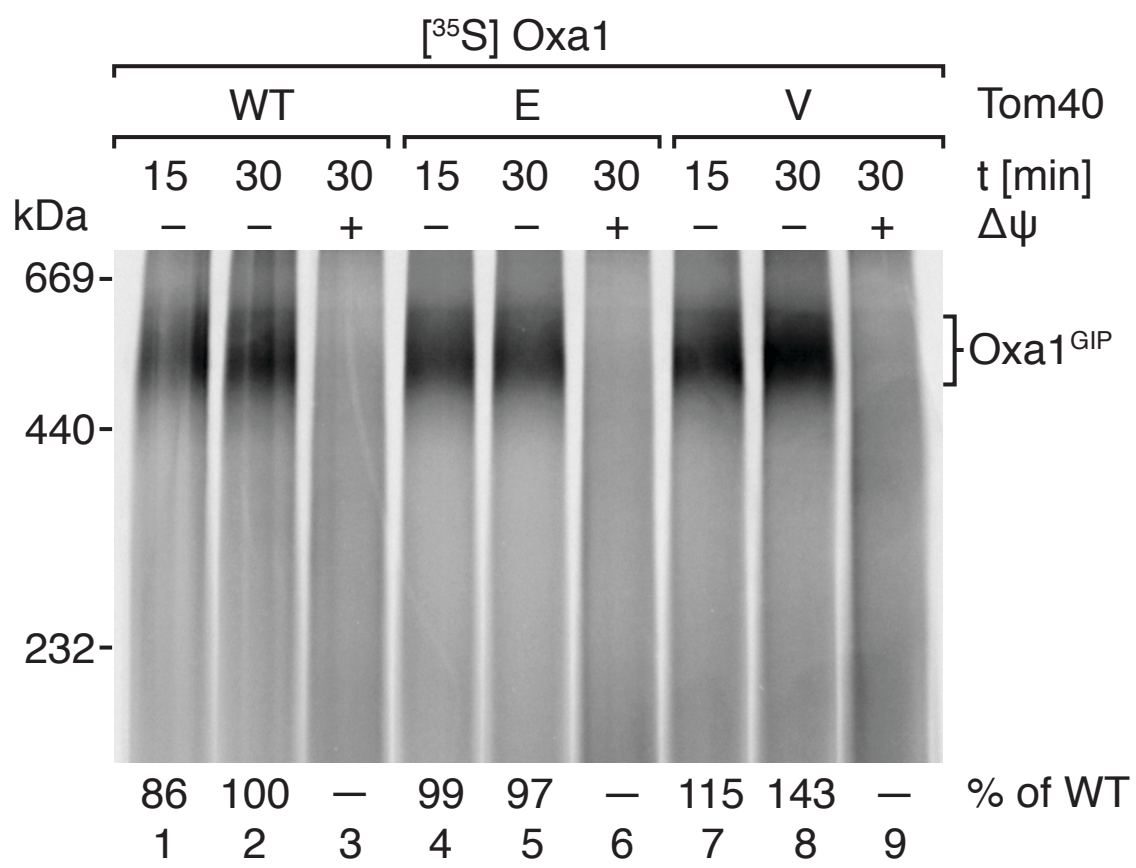

Figure 21. Presequence mediated TOM translocation analysis in Tom40 phospho-mutant mitochondria. (A) $\left[{ }^{35} \mathrm{~S}\right] \mathrm{Oxal}$ was imported with and without $\Delta \psi$ and analyzed via BN-PAGE and digital autoradiography. An Oxa1 ${ }^{\text {GIP }}$ intermediate autoradiograph is presented with the quantification presented below, corresponding to \% of wild-type at $30 \mathrm{~min}$. Image provided by Dr. Christian Schulz. 


\subsection{Investigating the functional relationship between the presequence-binding sites of Tom40 and Tom $22^{\mathrm{IMS}}$.}

Having established that a functional presequence-binding site within the Tom 40 channel exists, the question was raised as to its relationship with the nearby and well established presequence-binding site of Tom $22^{\mathrm{IMS}}$ (Frazier et al., 2003; Komiya et al., 1998; Moczko et al., 1997; Shiota et al., 2011). In order to address this issue, a series of double mutant yeast strains were created, corresponding to the previously established Tom40 T220 phospho-mutants, in conjunction with the established deletion of the Tom 22 IMS domain via the tom22-2 allele (Moczko et al., 1997). Specifically, competent tom $40 \Delta$ yeast containing either TOM40, tom $40^{E \text { mut }}$ or $\operatorname{tom} 40^{V}$ mut on a plasmid were transformed with a PCR-generated integration cassette, enabling the chromosomal deletion of the Tom22 IMS domain as previously published (Frazier et al., 2003; Moczko et al., 1997). These newly generated double mutant yeast strains were applied to non-fermentable solid media in a dilution growth test over a range of temperatures (Fig. 22A). As seen in the image below, the addition of the Tom22 IMS domain deletion had no effect on the growth of the Tom40 phospho-mutant strains.

Mitochondria were isolated from the newly created yeast strains and steady-state protein levels of selected mitochondrial-resident proteins were assessed via SDS-PAGE and Western-blotting (Fig. 22B). Careful analysis of the Western blot images revealed unaltered protein levels in the double mutant strains, and particularly, examined TOM complex constituents displayed equal protein amounts across all strains. As TOM subunits in all double mutant strains exhibited wild-type-like steady-state protein levels, the stability of the double mutant TOM complexes was assessed via digitonin solubilization, BN-PAGE and Western-blotting (Fig. 22C). Similarly to what was seen in the single Tom40 phospho-mutants (Fig. 18B), the examined TOM complexes as well as complexes III and IV of the respiratory chain from the double mutant strains were shown to migrate equally on BN-PAGE. However, in strains containing Tom22-2, the TOM complex was seen to travel slightly faster as compared to the wild-type TOM complex (Tom22-2 containing TOM $\sim 300 \mathrm{kDa}$, wild-type TOM $\sim 400 \mathrm{kDa}$ ), due to the deletion of the Tom 22 IMS domain. The difference in TOM BN-PAGE migration due to the deletion of the IMS domain of Tom22 is likely related to alteration in TOM containing digitonin micelles, as the IMS domain is less than $4 \mathrm{kDa}$ in size. 
Succinctly, the aforementioned growth, protein steady-state and TOM stability analyses demonstrate wild-type-like TOM characteristics, and allow for further functional presequence-specific import analysis to proceed without the hindrance of indirect effects.

A
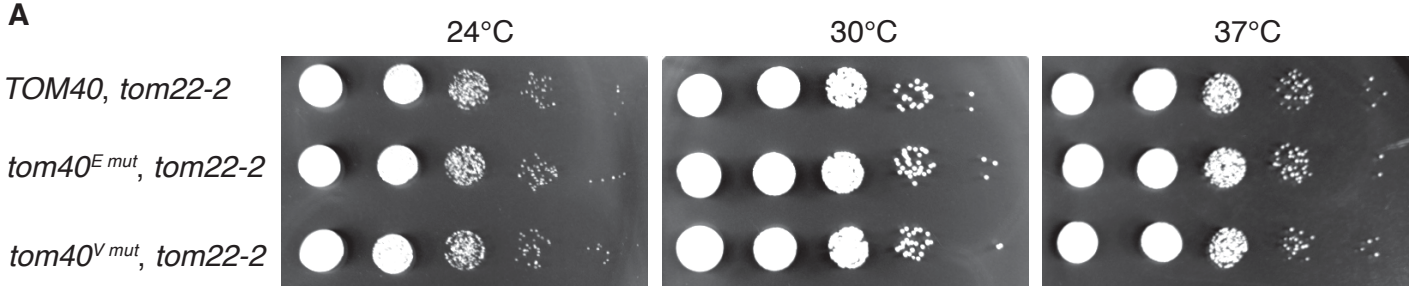

B

C
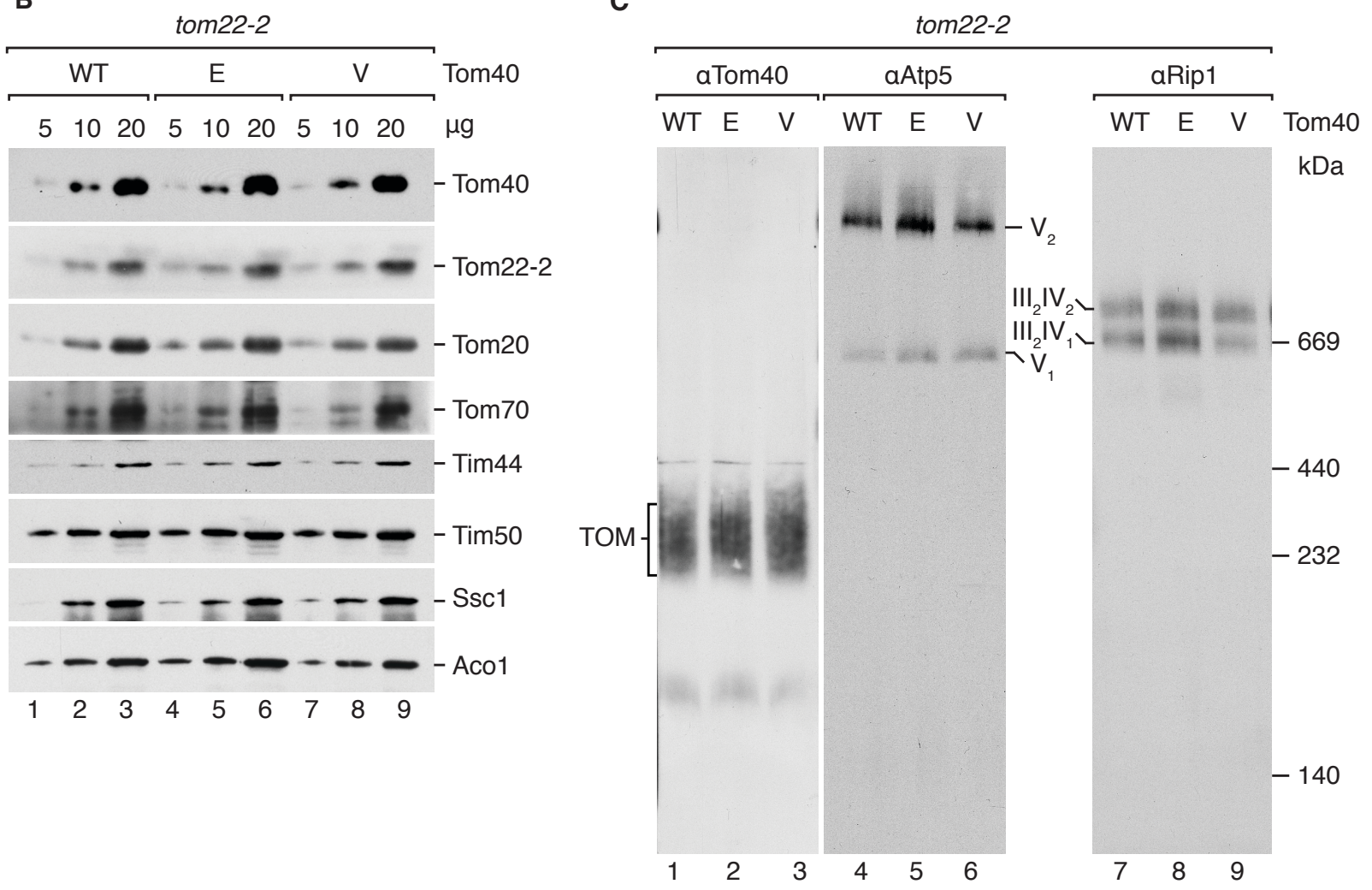

Figure 22. tom $40^{E m u t}$ and $t o m 40^{V m u t}$ alleles display unaffected growth, protein levels and TOM stability in the tom 22-2 background. (A) Serial dilutions (10-fold) of the tested strains were incubated at the indicated temperature for three days on non-fermentable medium. (B) Western blot analysis of steady-state protein levels in isolated mitochondria from the indicated strains via SDSPAGE, using specified antisera. (C) BN-PAGE Western blot analysis of steady-state complex levels using antisera for Tom40, Atp5 and Rip1 using $40 \mu \mathrm{g}, 15 \mu \mathrm{g}$ and $15 \mu \mathrm{g}$ isolated mitochondrial protein, respectively.

In order to investigate presequence mediated translocation over the TOM complex in the Tom40 phospho-mutants within the tom22-2 background, the Oxa1-TOM translocation intermediate assay was utilized, as previously performed in the Tom40 single mutants (Fig. 23). Contrary to the increase in TOM translocation kinetics seen in Tom $40^{\mathrm{V}}$ 
containing mitochondria within the wild-type background (Fig. 21), the deletion of the Tom22 IMS domain resulted in an approximately $25 \%$ drop in the Oxa1-TOM translocation intermediate, specifically in the Tom40 phospho-mutants.

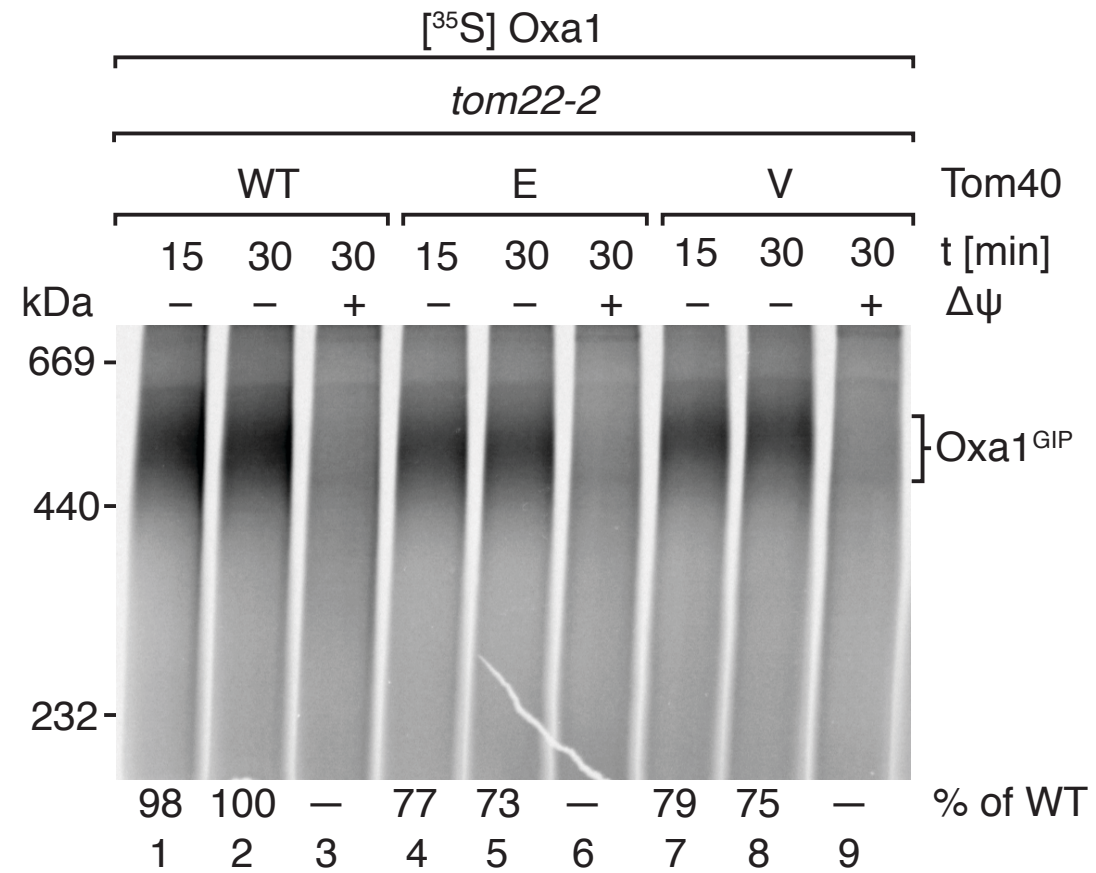

Figure 23. tom $40^{E}$ mut and $t o m 40^{V}$ mut alleles equally exhibit reduced TOM presequence translocation in the tom 22-2 background. $\left[{ }^{35} \mathrm{~S}\right] \mathrm{Oxa} 1$ was imported with and without $\Delta \psi$ as indicated into mitochondria isolated from the inferred strains and analyzed by BN-PAGE. An autoradiograph of the Oxa1 ${ }^{\text {GIP }}$ intermediate is displayed and quantification was preformed and presented as \% of wild-type at $30 \mathrm{~min}$. Image provided by Dr. Christian Schulz.

In summary, the analysis of Tom40 phospho-mutants in the tom 22-2 background, compared to the results of the functional analysis in the single Tom40 phospho-mutants, allowed for a defined set of conclusions to be drawn. First of which, Tom40 possesses a posttranslationally regulated presequence-binding site functionally involved in presequence mediated mitochondrial OM translocation. This evidence is seen in the specific reduction of presequence probe photo-adduct formation in mitochondria containing Tom $40^{\mathrm{E}}$, and conversely, presequence mediated import over the TOM complex is kinetically accelerated in $\mathrm{Tom} 40^{\mathrm{V}}$ mitochondria while exhibiting wild-type-like presequence probe photo-adduct formation. Nonetheless, both Tom40 phospho-mutants are uniquely sensitive to the deletion of the Tom22 IMS domain, allowing for the allocation of heightened presequence-related TOM translocation activity seen in Tom $40^{\mathrm{V}}$ 
mitochondria to the activities of the lone established trans-acting TOM presequencebinding domain, Tom $22^{\mathrm{IMS}}$. Attempting to better understand the possibly conflicting Tom40 phospho-mutant presequence interaction data, a detailed import analysis was performed at saturating conditions, pushing the translocation capacity of the TOM complex to maximal activity.

\subsection{Tom40 phosphorylation at $\mathbf{T} 220$ allows for the manipulation of import kinetics along the presequence pathway.}

Employing the use of the well characterized, artificial mitochondrial substrate $b_{2}(167)_{\Delta}$-DHFR, comprised of the first 167 residues from cytochrome $b_{2}$ (lacking the endogenous stop-transfer signal, hence the $\Delta$ ), combined with mouse DHFR, presequence mediated matrix-targeted import was examined in Tom40 phoshpo-mutants. The artificial substrate was expressed recombinant and purified via ion-exchange chromatography through a previously published protocol, allowing for the retention of import competency (Dekker et al., 1997). The use of a recombinant substrate, as opposed to previous import assays utilizing an $\left[{ }^{35} \mathrm{~S}\right] \mathrm{Met}$ reticulocyte lysate system, enabled the assessment of import under translocase saturating conditions.

As seen in Fig. 24A and B, $b_{2}(167)_{\Delta}$-DHFR was imported and processed by the matrix resident MPP (seen in Fig. 19A as i) and a presently elusive peptidase (seen in Fig. $24 \mathrm{~A}$ as $\left.\mathrm{i}^{*}\right)$ in the Tom40 phospho-mutants in a manner which enabled the kinetic resolution of presequence mediated matrix import. Examining the quantification of presequence import across the Tom40 phospho-mutant containing mitochondria, employing a fluorescence based Western blot detection method, opposing import kinetics was revealed. Tom $40^{\mathrm{V}}$-containing mitochondria displayed wild-type-like import kinetics during the initial linear import phase, however, these mitochondria appear to have remained in the linear phase for an extended period of time, resulting in an $\sim 25 \%$ increase in overall import capacity in the saturation phase of the import curve. Conversely, Tom $40^{\mathrm{E}}$-containing mitochondria exhibited an overall drop in presequence import, seen more prevalently in the early time points as the Tom $40^{\mathrm{E}}$ mutant mitochondria were able to approach wild-type levels at saturation. Taken together, the matrix-targeted presequence import phenotypes exhibited by the Tom40 phospho-mutants are supported by the aforementioned data, specifically, the reduction in $\mathrm{Tom} 40^{\mathrm{E}}$ presequence-binding as assessed by photo-crosslinking with the presequence probes. In the case of $T 0 m 40^{\mathrm{V}}$, the increase in TOM translocation kinetics, as monitored by the Oxa1-TOM translocation 
intermediate assay, resulted in an increase of the overall presequence import capacity of a matrix-targeted substrate.

A

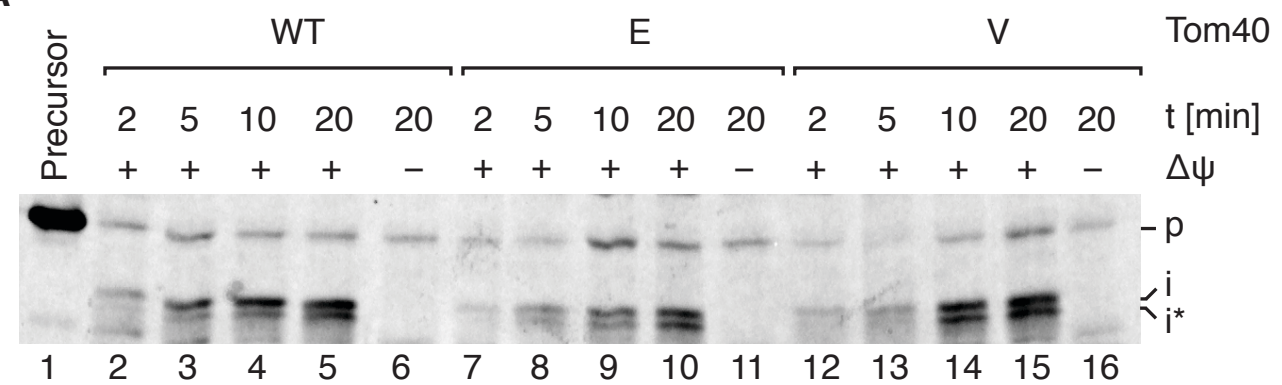

B

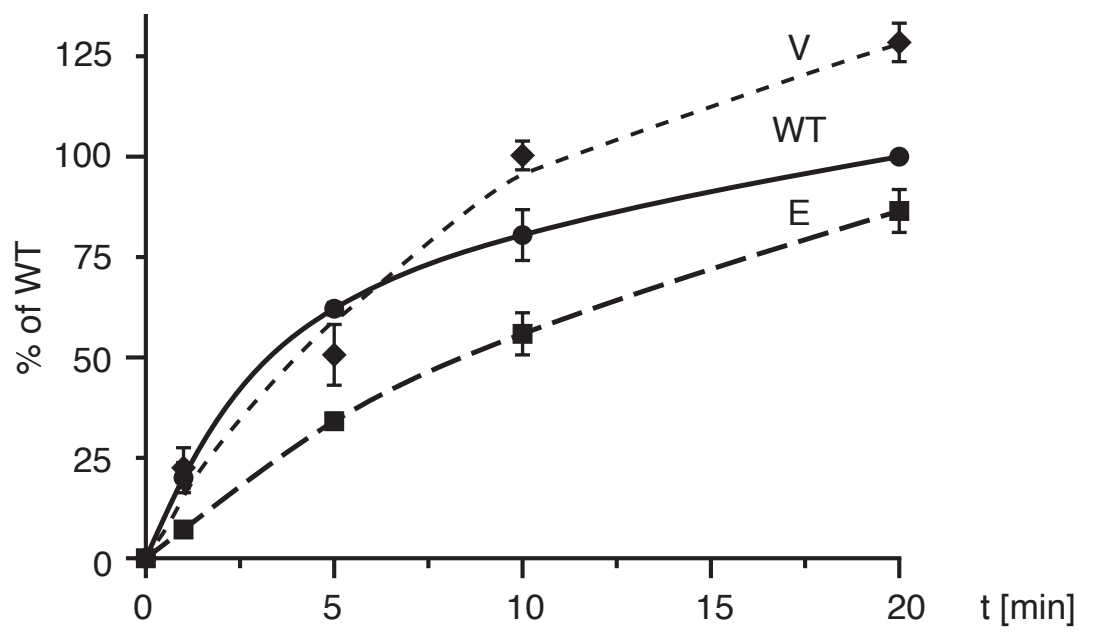

Figure 24. Tom $40^{\mathrm{E}}$ and $\mathrm{Tom}^{\mathrm{V}} \mathrm{V}^{\mathrm{V}}$ mitochondria exhibit opposing alterations in presequence mediated matrix import kinetics. (A) Purified $b_{2}(167)_{\Delta}$-DHFR was imported into isolated mitochondria from the indicated strains, proteinase $\mathrm{K}$ treated and analyzed via Western-blotting and fluorescence-based digital imaging using anti-DHFR antiserum. Precursor lane represents 15\% of the input. (B) Quantification of import reactions described in A. The processed intermediates are shown as $\%$ of wild-type at $20 \mathrm{~min}(\mathrm{n}=3, \mathrm{SEM})$. p, precursor. $\mathrm{i}$ and $\mathrm{i}^{*}$, processed intermediates.

Attempting to better understand the functional relationship between the presently characterized Tom 40 presequence-binding site and the single established late acting TOM presequence-binding site Tom $22^{\mathrm{IMS}}$, mitochondria from the previously created Tom 40 phospho-mutants in the tom22-2 background were used in the saturating $\mathrm{b}_{2}(167)_{\Delta}$-DHFR matrix translocation assay. As seen in Fig. 25, $b_{2}(167)_{\Delta}$-DHFR import was attenuated in 
both of the Tom40 T220 phospho-mutant strains, when compared to the corresponding wild-type. Careful examination of the import kinetics revealed that the Tom $40^{\mathrm{V}}$ mutant, as compared to the Tom $40^{\mathrm{E}}$ mutant and particularly the wild-type, suffered particularly from the loss of the Tom $22^{\mathrm{IMS}}$ domain, allowing for the gain of import capacity seen in Fig. 24 to be functionally linked to the TOM trans-acting presequence-binding site. This result parallels the findings from the Oxa1-TOM intermediate assay (Fig. 21 compared to Fig. 23), which also found the IMS domain of Tom22 to mediate the increase in TOM translocation kinetics seen in $\operatorname{Tom} 40^{\mathrm{V}}$.

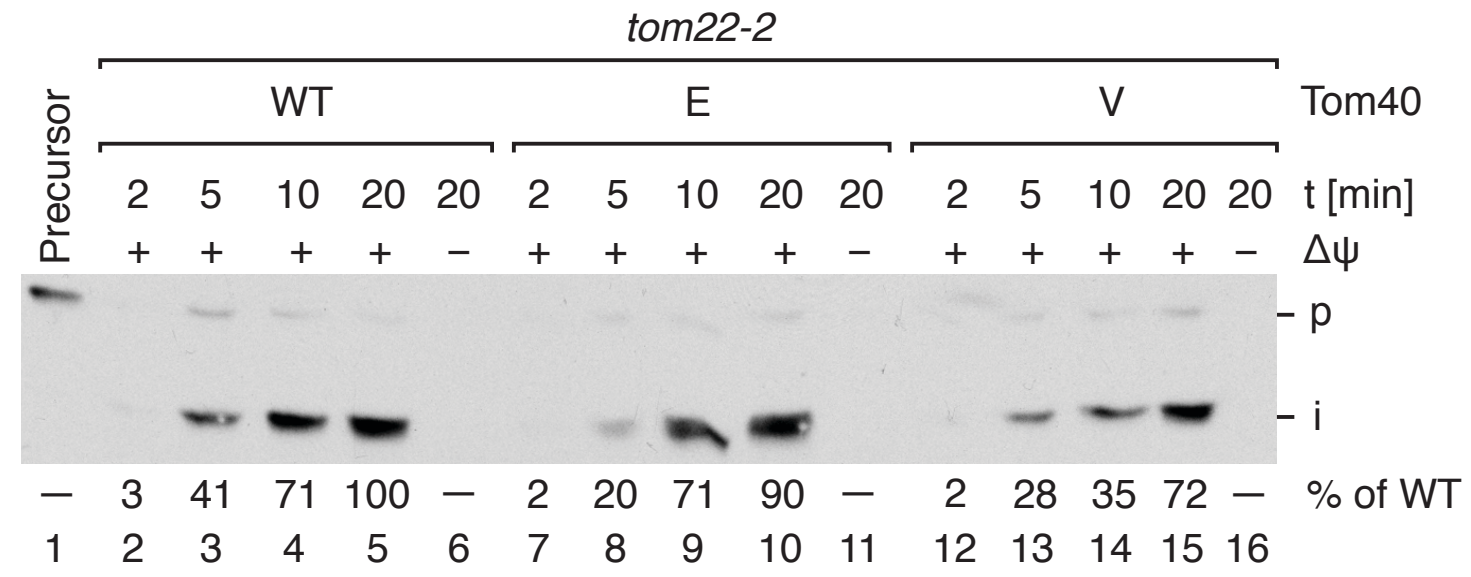

Figure 25. Tom $40^{\mathrm{E}}$ and $\operatorname{Tom}^{\mathrm{V}}{ }^{\mathrm{V}}$ mitochondria display sensitivity to the loss of Tom $22^{\mathrm{IMS}}$ in presequence mediated matrix import. Purified $b_{2}(167)_{\Delta}$-DHFR was imported into mitochondria isolated from Tom40 phospho-mutant strains in the tom 22-2 background, proteinase $\mathrm{K}$ treated and analyzed via Western-blotting, employing anti-DHFR antiserum. Precursor lane represents 15\% of the input. p, precursor. i, processed intermediate. 


\subsection{The characterization of a novel TIM23 interaction between Tim50 and Tim21.}

As presequence-containing precursors exit the OM TOM channel, they immediately come into contact with receptors of the presequence translocase while still stably associated with TOM (Albrecht et al., 2006; Chacinska et al., 2005; Mokranjac et al., 2005; Popov-Celeketić et al., 2008a). Specifically, both Tim21 and Tim50 have been observed to interact with either IMS residing TOM subunits or the presequence-containing substrate in complex with TOM. Moreover, these interactions are known to exhibit functional independence of the $\Delta \psi$, signifying the early actions of these IM bound presequence translocase receptors, proceeding presequence-induced TIM23 channel opening (Meinecke et al., 2006; Truscott et al., 2001).

The various sorting routes employed by the multi-faced presequence translocase demand its demonstrated highly dynamic nature (Chacinska et al., 2009; Dudek et al., 2013; van der Laan et al., 2010). These dynamic events have been observed throughout literature as a plethora of intra-translocase interactions (see section 1.3.1. for a detailed description), and are now known to be functionally linked to the active import of the heterogeneous class of presequence-containing substrates. Investigating key Tim50 interaction partners through its single cysteine residue, a chemical crosslinking approach was employed by applying a highly spatially restrictive cysteine active crosslinker (exhibits and active radius of $\sim 3 \AA$ ), namely $\mathrm{Cu}^{2+}$ (Lytovchenko et al., 2013). In this study, Tim21 (also containing a single endogenous cysteine residue, $\mathrm{C}^{128}$ ) was unequivocally identified to form a $\mathrm{Cu}^{2+}$-induced intermolecular disulfide-bond with Tim50 (published findings summarized in Fig. 26). Moreover, the findings by Lytovchenko et al. demonstrated the sensitivity of the Tim50-Tim 21 interaction to presequence addition, as the specific crosslinking adduct band disappeared with the preincubation of mitochondria with presequence peptide (modeled in Fig. 26B). 

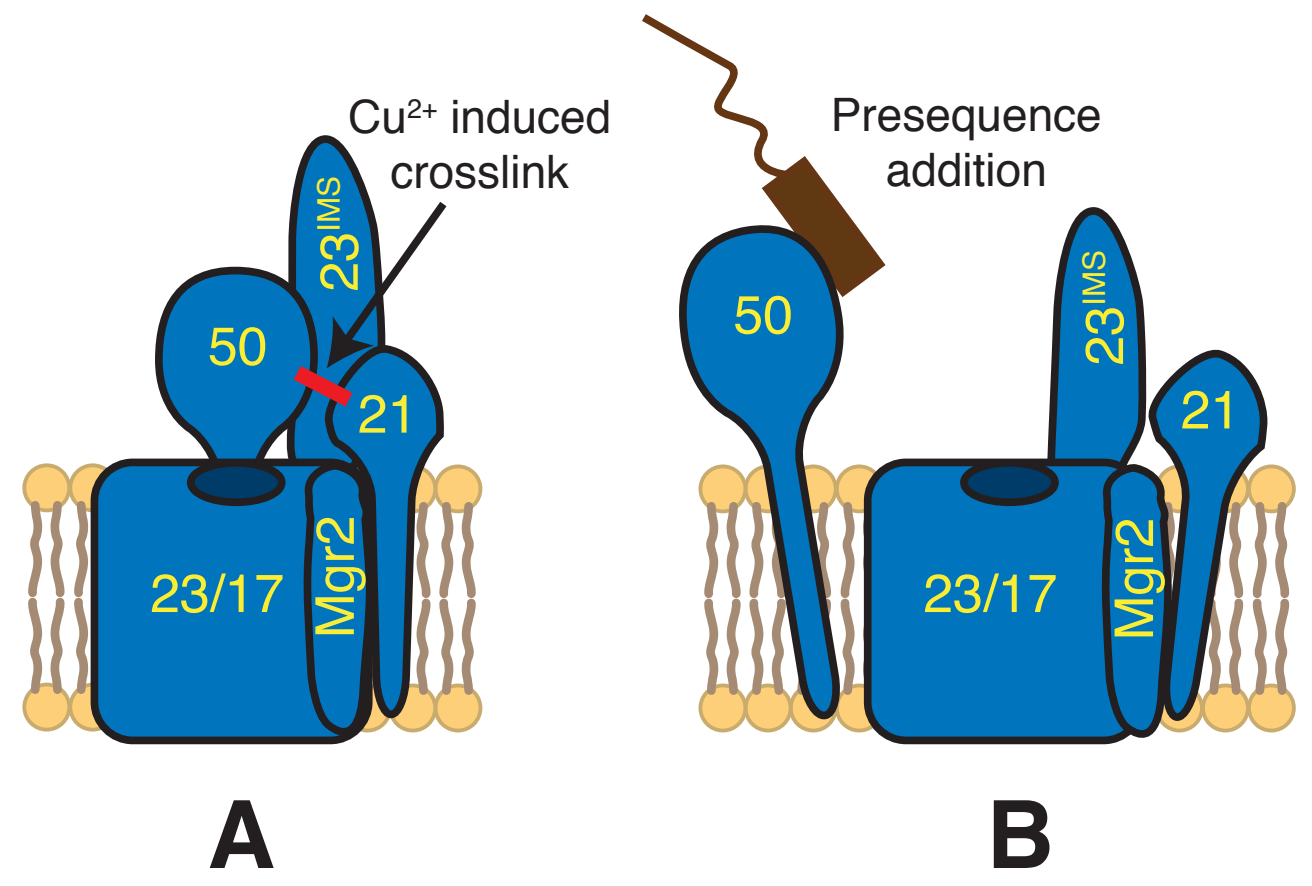

Figure 26. Tim21 can be crosslinked to $\mathrm{Tim50}$ in a presequence sensitive manner. (A) $\mathrm{Cu}^{2+}$ crosslinking in isolated mitochondria stabilizes the association of Tim50 to Tim21. (B) Following the addition of presequence peptide, the Tim50-Tim21 complex dissociates. Model summarizes previously published findings (Lytovchenko et al., 2013).

As Lytovchenko and colleagues had established the Tim50-Tim21 interaction in organello, the association was further investigated upon via examination within a defined in vitro system. The use of this unambiguous experimental set-up allowed for the investigation of the potential roles other TIM23 constituents instilled on the newly described Tim50-Tim 21 interaction, specifically the impact of the Tim $23^{\mathrm{IMS}}$ domain. Tim23 represented a key TIM23 component to be investigated in the in vitro system as it represents the only other presequence translocase subunit containing a functionally relevant IMS domain. Moreover, critical residues within Tim $23^{\mathrm{IMS}}$ responsible for its presequence import-conferring Tim50 interaction have been identified (GevorkyanAirapetov et al., 2009), namely ${ }^{70} \mathrm{YL}^{71}$. In order to achieve this, the IMS domains of Tim23 (both wild-type and YL70AA mutant), Tim50 and Tim21 were recombinantly expressed and purified.

$\operatorname{Tim} 21^{\mathrm{IMS}}$ was immobilized on resin and $\operatorname{Tim} 50^{\mathrm{IMS}}$ was added to the system at a steady concentration, while the amount of the third component, Tim $23^{\mathrm{IMS}}$ was titrated in. Bound Tim50 ${ }^{\mathrm{IMS}}$ was eluted and visualized via SDS-PAGE and Western-blotting (Fig. 27). The Tim $21^{\mathrm{IMS}}-\mathrm{Tim} 50^{\mathrm{IMS}}$ interaction was seen to occur in vitro without the addition of 
$\operatorname{Tim} 23^{\mathrm{IMS}}$ (seen in Fig. 27A, lane 2 compared to lane 6), however, the addition of Tim50 binding competent Tim $23^{\mathrm{IMS}}$ to the in vitro system was shown to increase Tim50 ${ }^{\mathrm{IMS}}$, $\mathrm{S}$ association with Tim $21^{\mathrm{IMS}}$ by over twofold (Fig. 27B). Moreover, as previously established by Gevorkyan-Airapetov and colleagues, the Tim50 binding incompetent mutant Tim $23^{\text {IMS }}$ construct (Gevorkyan-Airapetov et al., 2009) Tim $23^{\text {YL70AA }}$ was unable to initiate increased recovery of $\operatorname{Tim} 50^{\mathrm{IMS}}$ from the $\operatorname{Tim} 21^{\mathrm{IMS}}$ resin in the in vitro system. This finding signifies the requirement of Tim23's interaction with Tim50, in the construction of the Tim21Tim50 interaction. Finally, this notion is suggestive of tertiary complex formation with $\operatorname{Tim} 23$ acting as a bridge between Tim50 and Tim21, however, the possibility of an enzyme-like function of Tim23 ${ }^{\text {IMS }}$ altering Tim50's Tim21 interaction surface cannot be excluded.
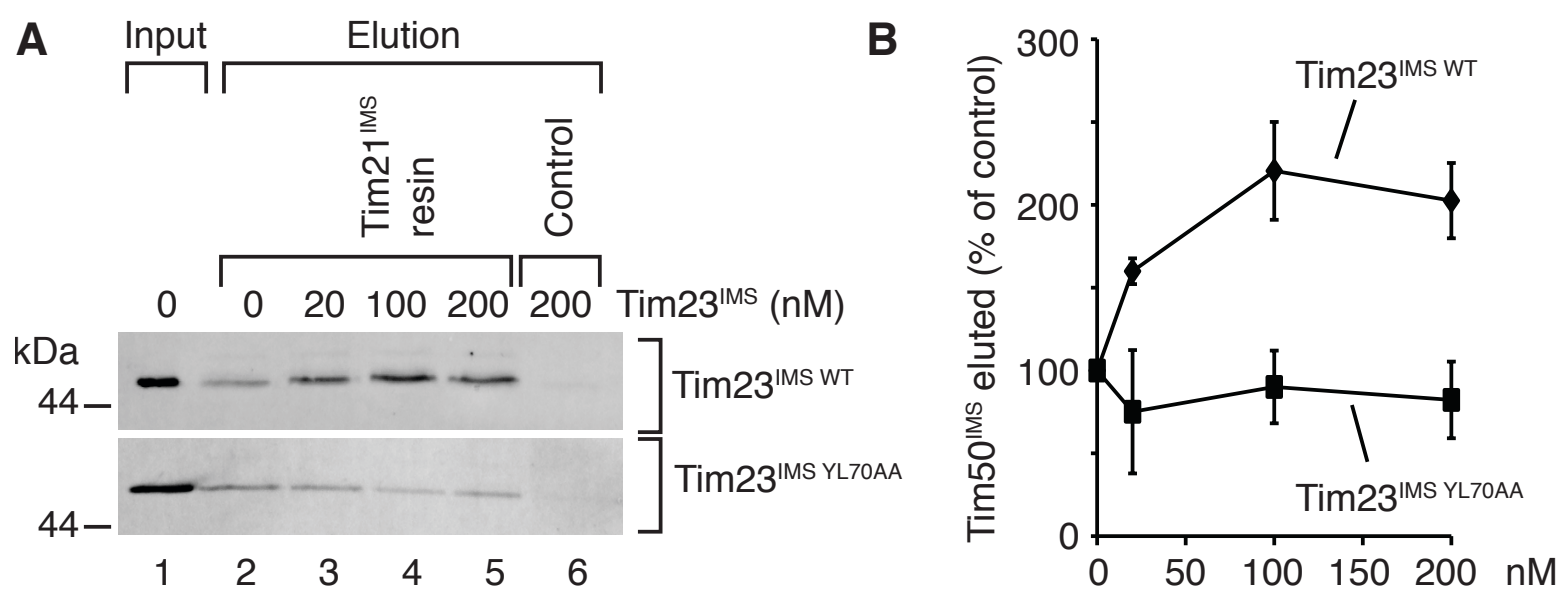

Figure 27. Tim $23^{\text {IMS }}$ establishes the Tim21-Tim50 interaction. (A) Tim $21^{\text {IMS }}$ was bound to CNBractivated Sepharose and mixed with $20 \mathrm{nM}$ Tim $50^{\mathrm{IMS}}$ in the presence of the indicated concentrations of $\operatorname{Tim} 23^{\text {IMS WT }}$ or Tim $23^{\text {IMS YL70AA }}$. Protein bound on the Tim $21^{\text {IMS }}$ resin was acidically eluted and analyzed by SDS-PAGE and Western-blotting using Tim50 antiserum. (B) Quantification of Tim50 ${ }^{\mathrm{IMS}}$ signal intensities, as in (A), presented as mean $\pm \mathrm{SEM}, \mathrm{N}=3$.

\subsection{Presequences trigger the dissociation of Tim50 from Tim 21 and prime the presequence translocase for translocation.}

As the previous assay indisputably established the interaction between Tim21, $\operatorname{Tim} 23$ and Tim50, the significance of this association in mitochondria was questioned. Specifically, what role the signal sequences had on the newly described interaction within the context of a primed vs. default state translocase within a mitochondrial setting. In order to investigate this concept, a presequence translocase priming assay was combined with immunoisolation of the TIM23 complex via Tim23 as well as Tim50. In detail, co- 
immunoprecipitation experiments were performed with Tim50 and Tim23 antisera utilizing digitonin-solubilized mitochondria that had previously been primed through the incubation in $\mathrm{pCox} 4$, or left in the default state via addition of Synb2 (similar in composition to $\mathrm{pCox} 4$, but fails to target substrates to mitochondria [Allison and Schatz, 1986]) or buffer incubation (Fig. 28). Importantly, this assay allowed for the detection of dynamic TIM23 subunits as the experimental set-up enables the discrimination between the primed and default state translocase. Investigation of the co-immunoprecipitation results importantly observed the highly significant (Fig. 28B, left panel) dissociation of Tim21 from Tim50 upon pCox4 addition as compared to the controls (Fig. 28A, lane 5 compared to lanes 4 and 6). This observation was validated by the fact that Tim50 and Tim23 were isolated in equal efficiencies across all mitochondrial pre-treatment conditions when the respective antiserum was used. Moreover, the dissociation of Tim21 from Tim50 was shown to occur at the presequence translocase and not in a free Tim21-Tim50 pool as the same statistically significant dissociation was observed when Tim23 antiserum was used. Of particular interest, Pam17, the established early TIM23 integrating PAM subunit presumed to be responsible for the subsequent association of the Pam16/Pam18 complex (Popov-Celeketić et al., 2008a; Schiller, 2009; van der Laan et al., 2005), was shown to exhibit reciprocal behavior when compared to Tim21 in both Tim50 and Tim23 coimmunoprecipitation experiments (Fig. 28). Taken together, these findings effectively linked presequence presentation in the IMS with the dissociation of the Tim23 mediated Tim21-Tim50 interaction at the presequence translocase resulting in the subsequent association of the early acting PAM subunit, Pam17, giving rise to a primed translocase. 

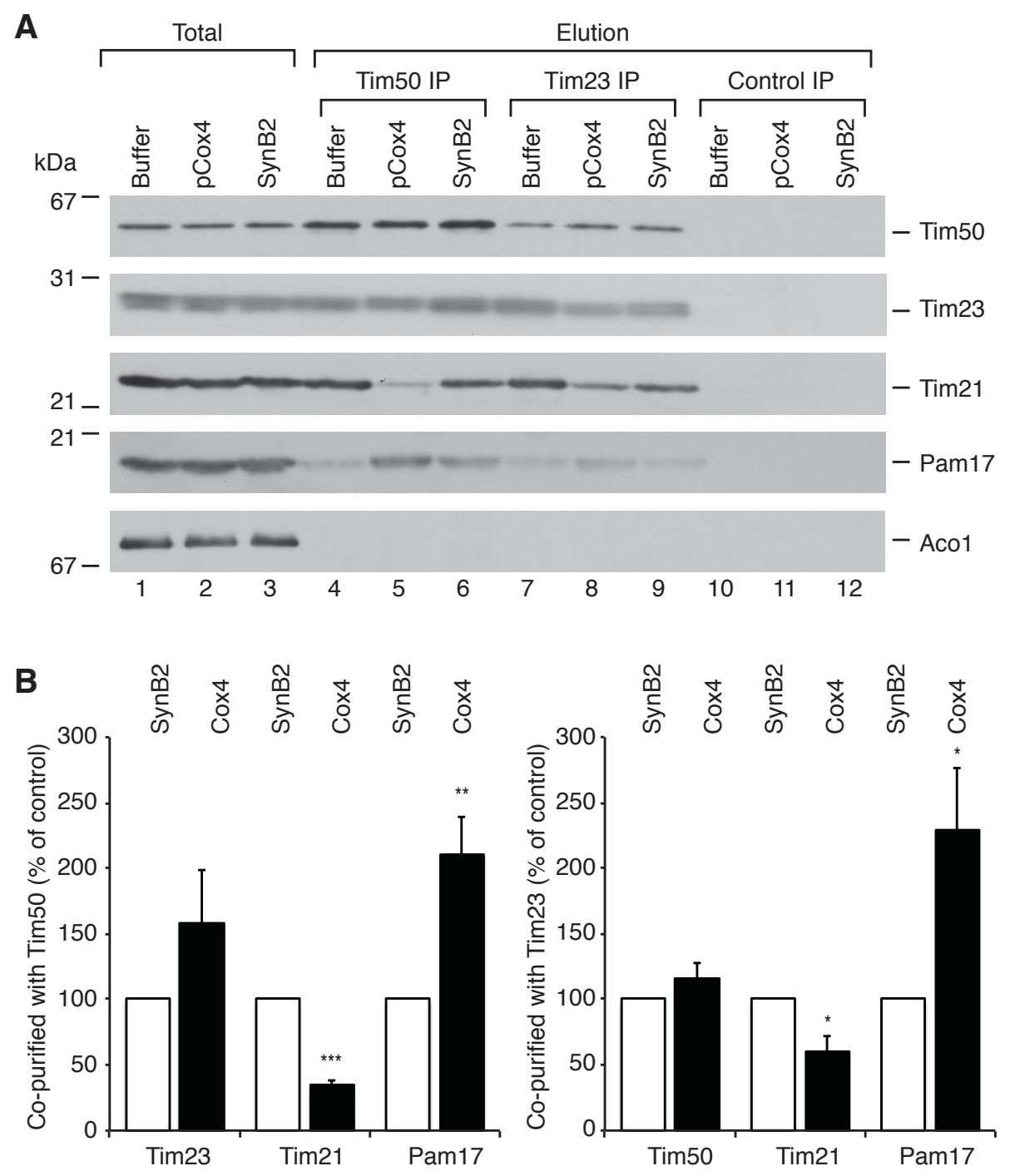

Figure 28. Tim50 and Tim21 dissociate upon signal sequence binding at the presequence translocase, leading to Pam17 association. (A) Co-immunoprecipitation was carried out with Tim50 or Tim23 antisera using digitonin-solubilized mitochondria preceded by the pre-treatment with pCox4, SynB2, or buffer. Control immunoprecipitation used a translocase distant antiserum. Bound proteins were acidically eluted and analyzed by SDS-PAGE and Western-blotting. Total represents $7 \%$ of the elution. (B) Seven independent co-immunoprecipitation experiments employing pCox 4 and SynB2 pre-treatment using Tim50 (left panel) or Tim23 (right panel) antisera as performed in (A) were quantified and normalized to precipitated Tim50 or Tim23, with the SynB2 peptide control set at $100 \%$. Data are presented as mean $\pm \mathrm{SEM}, \mathrm{N}=7$. Statistical significance was evaluated using a twosided t-test. *, $P<0.05 ; * *, P<0.01 ; * * *, P<0.0001$.

Further investigation into the presequence-triggered dissociation of Tim21 from Tim50 at the TIM23 complex questioned the significance of the established C-terminal Tim50 presequence-binding domain (Schulz et al., 2011). To investigate this issue, 
mitochondria were isolated from a strain in which TIM50 was placed under the control of the GAL1 promoter and carrying either a plasmid encoding $\operatorname{Tim}_{50} 0^{\mathrm{HA}}$ or Tim50 ${ }^{1-361-\mathrm{HA}}$. Before yeast were subjected to mitochondrial isolation the galactose was removed from the culture media, enabling the depletion of the genomically encoded Tim50, thereby allowing for functional integration of the particular plasmid encoded Tim50 variant into the presequence translocase.

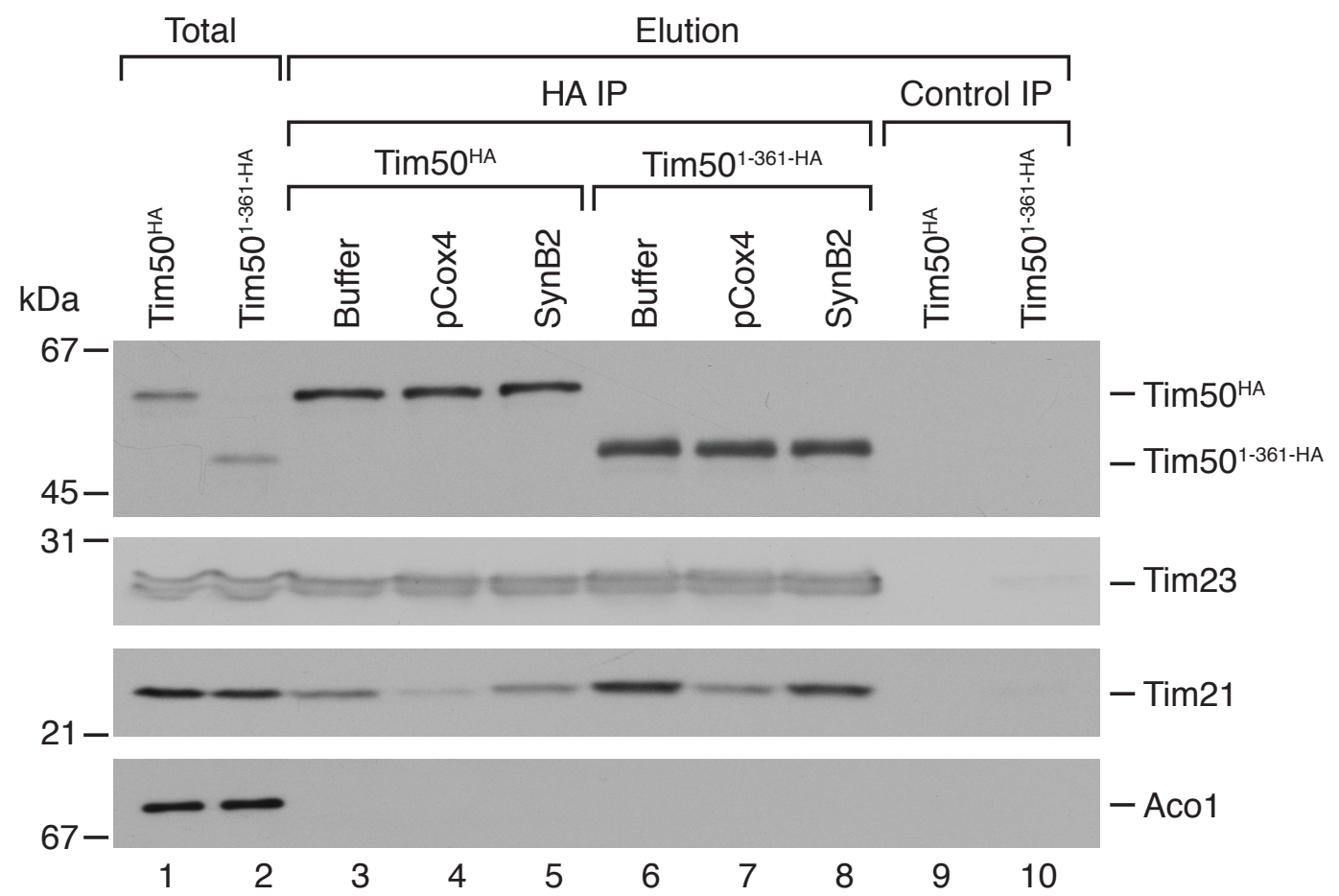

Figure 29. Tim50's presequence-binding domain is dispensable in the presequence-triggered dissociation of Tim21 from Tim50. Co-immunoprecipitation was carried out using anti-HA mouse monoclonal antibody and digitonin-solubilized mitochondria containing Tim50 ${ }^{\mathrm{HA}}$ and $\mathrm{Tim} 50^{1-361-\mathrm{HA}}$, pre-treated with pCox4, Synb2 or buffer. Bound proteins were acidically eluted and analyzed by SDSPAGE and Western-blotting. Total represents $7 \%$ of the elution. Anti-Myc mouse monoclonal antibody (9E10) was used as an immunoprecipitation specificity control.

As seen in Fig. 29, HA resin successfully isolated both Tim50 variants to equal degrees across all mitochondrial pre-treatment conditions as similar amounts of $\operatorname{Tim} 50^{\mathrm{HA}}$ or Tim50 $0^{1-361-\mathrm{HA}}$ were recovered. Moreover, as demonstrated by the unvaried levels of $\operatorname{Tim} 23$ co-immunoprecipitation, both $\operatorname{Tim} 50^{\mathrm{HA}}$ and $\operatorname{Tim} 50^{1-361-\mathrm{HA}}$ equally incorporate into the TIM23 complex. Importantly, unvaried levels of relative Tim21 dissociation from both $\operatorname{Tim} 50^{\mathrm{HA}}$ and $\mathrm{Tim} 50^{1-361-\mathrm{HA}}$ were observed, indicating that the C-terminal presequence- 
binding domain of Tim50 is indeed dispensable for the previously established Tim50Tim21 dynamics in the context of presequence translocase priming. 


\section{Discussion}

\subsection{The Tom40 channel recognizes presequences and augments their translocation kinetics in route to the inner membrane.}

The data described in this study confirms numerous publications in which Tom40 was speculated to be a presequence interactor (Ahting et al., 2001; Gabriel et al., 2003; Gaikwad and Cumsky, 1994; Gessmann et al., 2011; Gordon et al., 2001; Hill et al., 1998; Juin et al., 1997; Kanamori et al., 1999; Künkele et al., 1998; Mahendran et al., 2012; Rapaport et al., 1998a; 1998b; Sherman et al., 2006; Stan et al., 2000). Specifically, the data presented here have effectively mapped the presequence-binding regions of Tom40 to two locations residing on opposite ends of the $\beta$-barrel. Moreover, an extensive literature analysis has confirmed previous speculations as to Tom40 regions believed to be actively involved in presequence association (see section 4.2. for details). Of particular interest, an established phosphorylation site (T220) (Schmidt et al., 2011) was seen to reside in the middle of one of the identified presequence interaction regions. Investigation of this site utilized the previously established targeted mutagenesis phospho-mimicking method (Gerbeth et al., 2013; Schmidt et al., 2011), entailing the creation of yeast phosphomimetic (T220E) and phospho-block (T220V) Tom40 mutants. Contrary to previous random attempts at mutating presequence-specific residues (Gabriel et al., 2003; Sherman et al., 2006), the novel in vivo $\mathrm{Tom}_{40}^{\mathrm{E}}$ and $\mathrm{Tom} 40^{\mathrm{V}}$ mutants presented unaltered TOM stability and functionality in regards to imported non-presequence related substrates. Moreover, the chosen method of presequence-binding site identification allowed for the subsequent conformation, through the very same presequence probe photo-crosslinking approach. In short, initial phospho-mutant analysis enabled both the conformation of the mapped presequence-binding domain and the involvement of posttranslational regulation within the binding pocket via a presently elusive kinase and phosphatase network.

Investigation of presequence mediated TOM translocation exposed altered presequence mediated translocation kinetics, with mitochondria containing $T o m 40^{\mathrm{V}}$ displaying faster $\mathrm{OM}$ translocation kinetics. This modification resulted in an increased TOM binding capacity for the presequence-containing substrate, observed as a stable TOM-substrate intermediate under conditions in which the $\Delta \psi$ as depleted. Moreover, this particular phosphorylation-mediated fine-tuning translocation mechanism, almost certainly residing within a much larger regulatory network, was shown to be functionally dependent 
upon the lone established trans-acting presequence-interacting domain, namely Tom $22^{\mathrm{IMS}}$. The strict dependence upon Tom $22^{\mathrm{IMS}}$ observed within the Tom 40 presequence-active site under question provides intriguing evidence of subunit cooperation within the TOM complex. Thus far, presequence-specific TOM subunit cooperation has been limited to the cytosolically exposed receptors (Shiota et al., 2011; Yamano et al., 2008), and therefore presents fresh insight into the thus far functionally uncharted IMS face of the TOM complex.

Attempting to achieve a great depth of understanding of the newly uncovered Tom40 presequence import regulatory groove, saturating import analysis was performed in the Tom40 phospho-mutants, allowing for matrix import to be assessed as a whole. Captivatingly, a phosphorylation reminiscent phenotype was observed within the mutants, as $\mathrm{Tom} 40^{\mathrm{E}}$ - and $\mathrm{Tom} 40^{\mathrm{V}}$-containing mitochondria demonstrated presequence import kinetics flanking that of the wild-type. Comparing both strains to the corresponding wildtype mitochondria, Tom $40^{\mathrm{V}}$ mitochondria exhibited an increased capacity for presequence import, as was seen in the aforementioned TOM-presequence substrate intermediate assay, and $\mathrm{Tom} 40^{\mathrm{E}}$ mitochondria displayed attenuated kinetics. One would assume that under wild-type conditions, a mixed phosphorylation population of Tom40 T220 exists; therefore it should reflect an intermediate phenotype when compared to the two extremes, mimicked here by the phospho-mutants. This phospho-mutant phenotype behavior has been previously seen to exist within the TOM complex (Gerbeth et al., 2013).

Finally, utilizing the saturating matrix-targeted presequence import assay within the Tom40 phospho-mutants in the tom22-2 background, a functional link was observed between the two presequence-active sites. Both $\mathrm{Tom}_{40}{ }^{\mathrm{E}}$ and $\mathrm{Tom} 40^{\mathrm{V}}$ mitochondria displayed heightened sensitivity to the genetic disruption of the Tom22 IMS domain. This finding closely paralleled the results of the presequence-specific TOM-intermediate translocation assay, allowing for the gain of presequence import function seen in the $\mathrm{Tom} 40^{\mathrm{V}}$ mutant to be attributed to a functional relationship with Tom $22^{\mathrm{IMS}}$. Additionally, the reduction in TOM translocation when monitored specifically is seen to lead to an overall reduction in matrix import when the presequence pathway is assessed as a whole.

\subsection{Presequence association at the TOM complex, Tom40's role}

The TOM complex is considered to be the master mitochondrial traffic regulator since all cytosolically translated substrates that are addressed beyond the outer membrane 
must enter the mitochondrion via TOM. An overwhelming majority of these substrates are guided through the outer membrane by a series of presequence-binding domains embedded in various presequence-active TOM subunits. Tom40 has time and time again throughout literature been proposed to be one of these presequence-active subunits (Baker et al., 1990; Gabriel et al., 2003; Gaikwad and Cumsky, 1994; Gessmann et al., 2011; Gordon et al., 2001; Hill et al., 1998; Kanamori et al., 1999; Mahendran et al., 2012; Rapaport et al., 1997; 1998a; Sherman et al., 2006; Stan et al., 2000; Vestweber et al., 1989), yet no study has delineated the causal relationship between Tom40 presequence-binding and its role in presequence mediated import. A major contributor to this disparity is the absence of Tom40 structural data, however, recent publications have validated the mouse VDAC based homology model and have given new meaning to the interpretation of primary sequence interaction data (Gessmann et al., 2011; Qiu et al., 2013). However, conclusive data regarding the orientation of the $\beta$-barrel in the membrane is still elusive and represents an attractive area for further investigation.

In section three of this study, efforts were made to identify presequence-interacting regions of Tom40, which resulted in the identification of three interacting peptides. Placing the Tom 40 residues of interest within the previously validated Tom 40 homology model (Qiu et al., 2013), two sites were apparent, localized to opposite ends of the $\beta$-barrel. This finding meshed seamlessly with the existing literature as Tom40 was previously proposed to hold two separate cis and trans presequence interaction sites through experimentally demonstrated differential salt sensitivity (Kanamori et al., 1999; Mayer et al., 1995). One of the two mapped presequence-interacting regions was defined to a higher degree due to the identification of multiple photo-crosslink containing peptides within the immediate vicinity. Interestingly, key presequence-specific residues or regions identified by previous works are seen to reside within or in the surrounding area of one of the two mapped presequence-binding locations.

Gabriel and colleagues screened a library of mutant TOM40 alleles for growth phenotypes over a wide range of temperatures and carbon sources and identified a presequence-specific sorting mutant, W243R (Gabriel et al., 2003). Although this mutant clearly exhibited compromised TOM stability, the presequence pathway was shown to be selectively affected. Importantly, the observed import phenotype can now be given structural backing, as the mutation resides within the $\beta$-sheet that flanks the newly characterized presequence-interacting region (Fig. 16, I and II). Sherman and others 
generated several mutants corresponding to conserved stretches and identified a N. crassa strain demonstrating attenuated presequence mediated import while leaving the carrier pathway unaffected (referred to as $\Delta 64$ ) (Sherman et al., 2006). However, similar to the findings in the aforementioned study, TOM stability was significantly compromised. Nevertheless, the presequence selectivity can be explained via the current presequence mapping data, as the stretch of residues omitted are located within the $\beta$-sheet attached to the identified presequence-interacting loop containing $\mathrm{M}^{94}$ (Fig. 16, III) in S. cerevisiae.

A recent publication by Gessmann and colleagues, examining Tom40's unique structural elements across a wide range of species, identified a well conserved polar groove within the B-barrel's interior (Gessmann et al., 2011). Captivatingly, this polar groove was not found in other OM ß-barrel proteins of high sequence identity, namely Por1 (known as VDAC in higher eukaryotes). This area of interest, corresponding to B-sheets 8-11, perfectly coincides with the presequence-interacting region mapped and functionally characterized in the present study. Collectively, the presequence-interacting regions described here are in complete agreement with previously published data and impart new meaning to prior observations.

\subsection{Regulation of mitochondrial import via posttranslational modification at the TOM complex.}

Initial global-scale phosphoproteome studies in baker's yeast ( $S$. cerevisiae) identified several phosphorylation sites within mitochondrial proteins, albeit the functional significance of the identified regulation points remained unknown (Albuquerque et al., 2008; Chi et al., 2007; Gnad et al., 2009; Holt et al., 2009; Li et al., 2007), yet in 2011, the long-elusive functional link between cytosolic kinase activity and mitochondrial import regulation was characterized (Schmidt et al., 2011). Schmidt and others utilized an exhaustive mass spectrometry approach to define the TOM phosphoproteome. In this trailblazing publication the authors functionally linked the actions of cytosolic kinases to the global regulation of mitochondrial import. Creatine kinase 2 (CK2) was shown to phosphorylate both Tom 22 and Mim1, bringing about increased steady-state TOM complex levels, allowing for a higher flux of mitochondrial traffic through the OM main entry gate. Moreover, protein kinase A (PKA) was observed to phosphorylate Tom70 particularly when yeast were cultured in non-fermentable media, resulting in the reduction of its receptor activity and the concurrent reduction in metabolite carrier import not required in non-respiratory conditions. Finally, for the first time, Schmidt et al. identified 
multiple Tom40 phosphorylated residues including the residue under investigation in the present study, T220. Using their newly established in vitro phosphorylation system, as in silico kinase prediction had failed to produce any viable results, an attempt was made to identify the kinase responsible for the phosphorylation of Tom40 at T220. However, the Tom40 phosphorylation signal of the recombinant phospho-mimetic mutant (T220E) was unphased upon the addition of PKA, CK1, MAPK and cyclin-dependent kinase 2 (CDK2), which prevented the assignment of a particular kinase (Schmidt et al., 2011).

Adding to the previously established mitochondrial import posttranslational regulatory mechanism at the TOM complex, Gerbeth and others further defined an intricate Tom22 centered TOM phosphorylation-based regulatory system (Gerbeth et al., 2013). In their study, glucose-induced signaling was correlated with TOM complex import regulation through CK1 mediated phosphorylation of the cytosolic Tom22 precursor (at a CK2 distinct residue), which brought about an increase in Tom22 and Tom20 import. Conversely, PKA's actions on the Tom 22 precursor resulted in its reduction of import. Collectively, the aforementioned studies showcase the existence of a phosphorylationbased import regulatory system at the TOM complex and validate the use of the presently employed phospho-mimetic point mutants in the biochemical characterization of posttranslational regulation at the TOM complex.

\subsection{Tom40's roles within the context of the posttranslationally mediated TOM import regulatory system.}

Having identified two presumptive presequence-interacting Tom40 surfaces, efforts were made to pinpoint various key residues in order to confirm and biochemically examine the newly defined presequence-interacting sites. Careful examination of the binding grooves uncovered the existence of a previously identified posttranslationally modified residue (T220) positioned in the middle of one of the newly identified binding surfaces (Schmidt et al., 2011). Through the use of previously validated, targeted phospho-active point mutations (Gerbeth et al., 2013; Schmidt et al., 2011), one of the presequenceinteracting regions revealed by mass spectrometry was confirmed in organello and functionally characterized within the context of presequence mediated import.

Integrating previously published knowledge of presequence mediated OM translocation via the TOM complex with the experimentally obtained insight gained in the present study, the following mechanism is proposed, schematically depicted in Fig. 30. (i) cytosolically translated mitochondrial-targeted, presequence-containing substrates are first 
captured by Tom20 via hydrophobic interactions (Abe et al., 2000; Brix et al., 1997); (ii) the presequence then forms a trimetric complex with Tom receptors Tom 22 and Tom 20 as it nears the TOM channel entrance (Shiota et al., 2011; Yamano et al., 2008); (iii) entering the Tom40 channel of the TOM complex, the presequence is guided to the cis-binding site within the $\beta$-barrel (mapped in the present study) with the assistance of Tom5 (Dietmeier et al., 1997; Kanamori et al., 1999; Mayer et al., 1995; Rapaport et al., 1997); (iv) the presequence is then pulled to the trans face of the $\beta$-barrel via its affinity to the Tom 40 trans-binding site, comprised of a Tom40 resident groove as well as the IMS domain of Tom22 (mapped and biochemically illustrated in the present study) (Chacinska et al., 2003; Frazier et al., 2003; Moczko et al., 1997) or if Tom40 T220 is phosphorylated, the presequence is then weakly attached to the trans face of the $\beta$-barrel via the now weakened interaction with the Tom40 trans-binding site and concomitantly is mildly anchored to the IMS domain of Tom22 (experimental portrayed in the present study); (v) with the presequence anchored at the trans-face of the TOM complex, its is presented to the primary IM bound presequence translocase receptor Tim50 (Schulz et al., 2011).

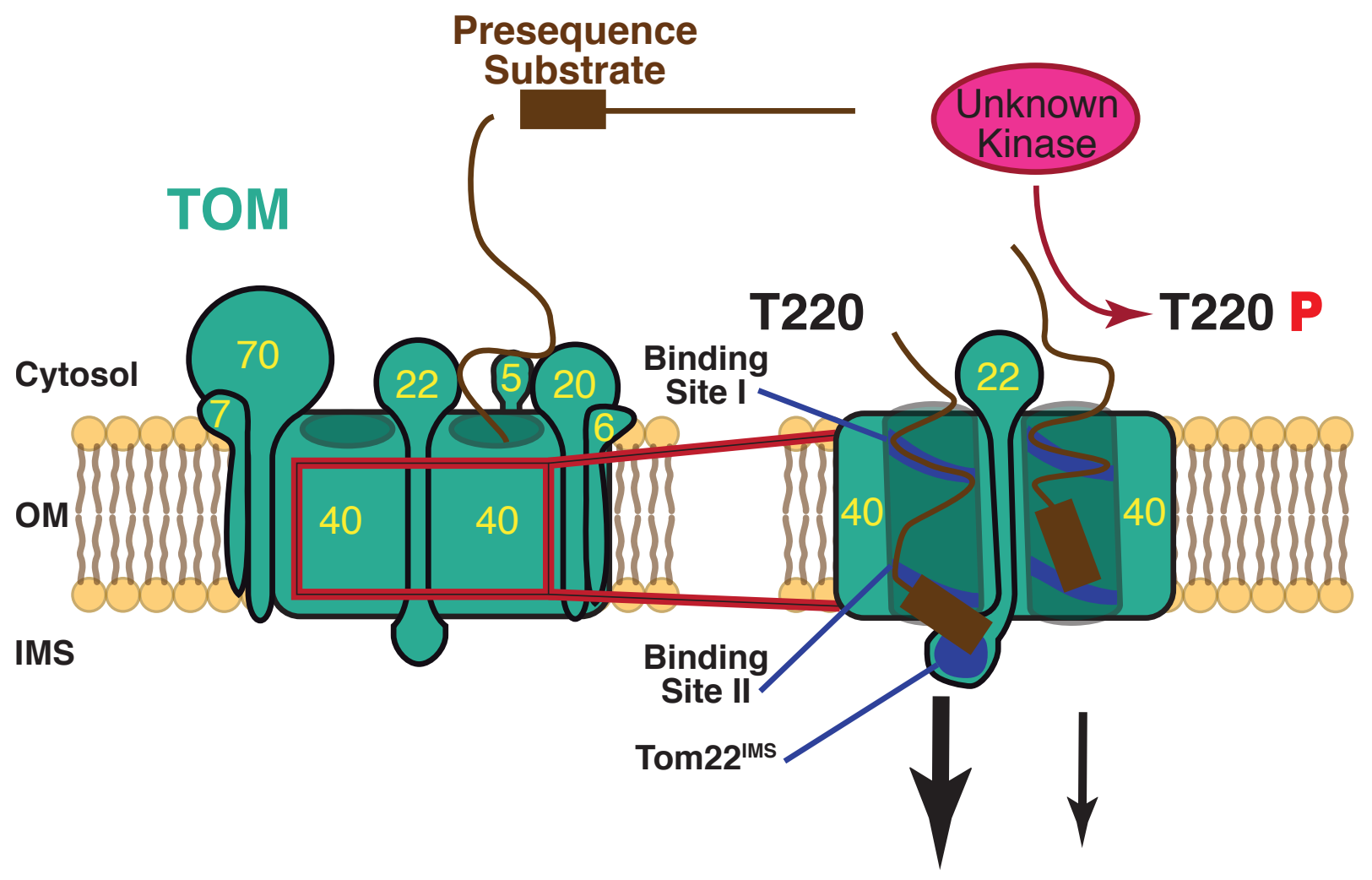

Figure 30. Schematic representation of presequence passage through Tom40 of the TOM complex. The TOM complex is illustrated on the left, depicting presequence mediated import guided by Tom20, Tom 22 (cytosolic domain), Tom5 and Tom40. On the right side, a cutout view of the 
TOM complex is presented, highlighting the Tom40 $\beta$-barrel presequence-binding domains (shown in blue) which were revealed in the study, as well as the intermembrane space (IMS) domain of Tom 22. Tom40 T220 phosphorylation by an elusive kinase functionally uncouples Tom $22^{\text {IMS }}$ from positive presequence-interaction and weakens Tom40's grip on the translocating presequence. OM, outer membrane.

In conclusion, Tom40 is identified in this study to serve a major role in OM presequence passage and is presented to function within a posttranslationally mediated presequence import TOM regulatory system.

\subsection{The dissociation of the Tim23 coordinated Tim21-Tim50 interaction is functionally linked to presequence translocase priming.}

The presequence translocase is a uniquely competent inner mitochondrial membrane translocase as it is versed in matrix translocation, IM integration as well as the recently demonstrated combination thereof (Bohnert et al., 2010). Enabling the accomplishment of this tall order, the TIM23 complex has been characterized to be present in two functionally distinct isoforms, namely Tim $23^{\text {SORT }}$ and Tim $23^{\text {MOTOR }}$ (see section 1.3.1.2. for a detailed description). The exact series of dynamic events required to convert a SORT TIM23 to a MOTOR TIM23 is not presently known, however, the TIM23 complex has been demonstrated on numerous occasions to exhibit functionally relevant intricate dynamic subunit exchange events described in depth within section 1.3.1. of this work.

Thus far, key publications have detailed some of these dynamic events and have concurrently begun to decipher the individual subunit exchanges in the context of an active presequence translocase (Alder et al., 2008; Chacinska et al., 2005; Gevorkyan-Airapetov et al., 2009; Marom et al., 2011; Mokranjac et al., 2009; Popov-Celeketić et al., 2008a; Popov-Čeleketić et al., 2011; Schulz et al., 2011; van der Laan et al., 2005; 2007). However, many critical junctions are still poorly understood, specifically what are the events that take place when the substrate is in transit from the trans side of the $\mathrm{OM}$ to the cis side of the IM? In this study, the dynamic events instilled upon the presequence translocase by an inbound signal sequence were investigated; enabling IMS subunit exchanges to be linked to IM/matrix localized translocase-priming events. 


\subsection{A presequence priming model of matrix import by the presequence translocase}

Placing the mechanistic knowledge gained in the current study within the confines of firmly established translocation-driving presequence translocase dynamic actions, the following mechanistic model of TIM23-mediated matrix import is advocated (schematic presentation portrayed in Fig. 31): (Fig. 31 - A) the default or inactive TIM23 complex is characterized by the core subunits of the presequence translocase Tim23, Tim17 and Mgr2, bound by the Tim 23 coordinated Tim50-Tim 21 complex, with the IMS domain of Tim50 serving to prevent TIM23 channel opening (Meinecke et al., 2006); (B) upon presequence emergence from the TOM channel, it is captured by Tim50's C-terminal presequence binding domain while still associated with the TOM complex (Schulz et al., 2011), triggering the release of the intra-TIM23 Tim50-Tim21 interaction; (C) Tim21 is released from the TIM23 complex upon Tim50-Tim23 interaction (Gevorkyan-Airapetov et al., 2009), and the presequence is handed-off to the IMS domain of Tim23 with the concurrent recruitment of Pam17, yielding a matrix import primed translocase; (D) the TIM23 channel is opened through a presequence and $\Delta \psi$ dependent mechanism (Truscott et al., 2001) together with the commencement of the force-conferring cyclic actions of the ATPdependent PAM complex through the dynamic association of PAM subunits with the presequence translocase (Popov-Celeketić et al., 2008a; Schiller, 2009; van der Laan et al., 2005). 

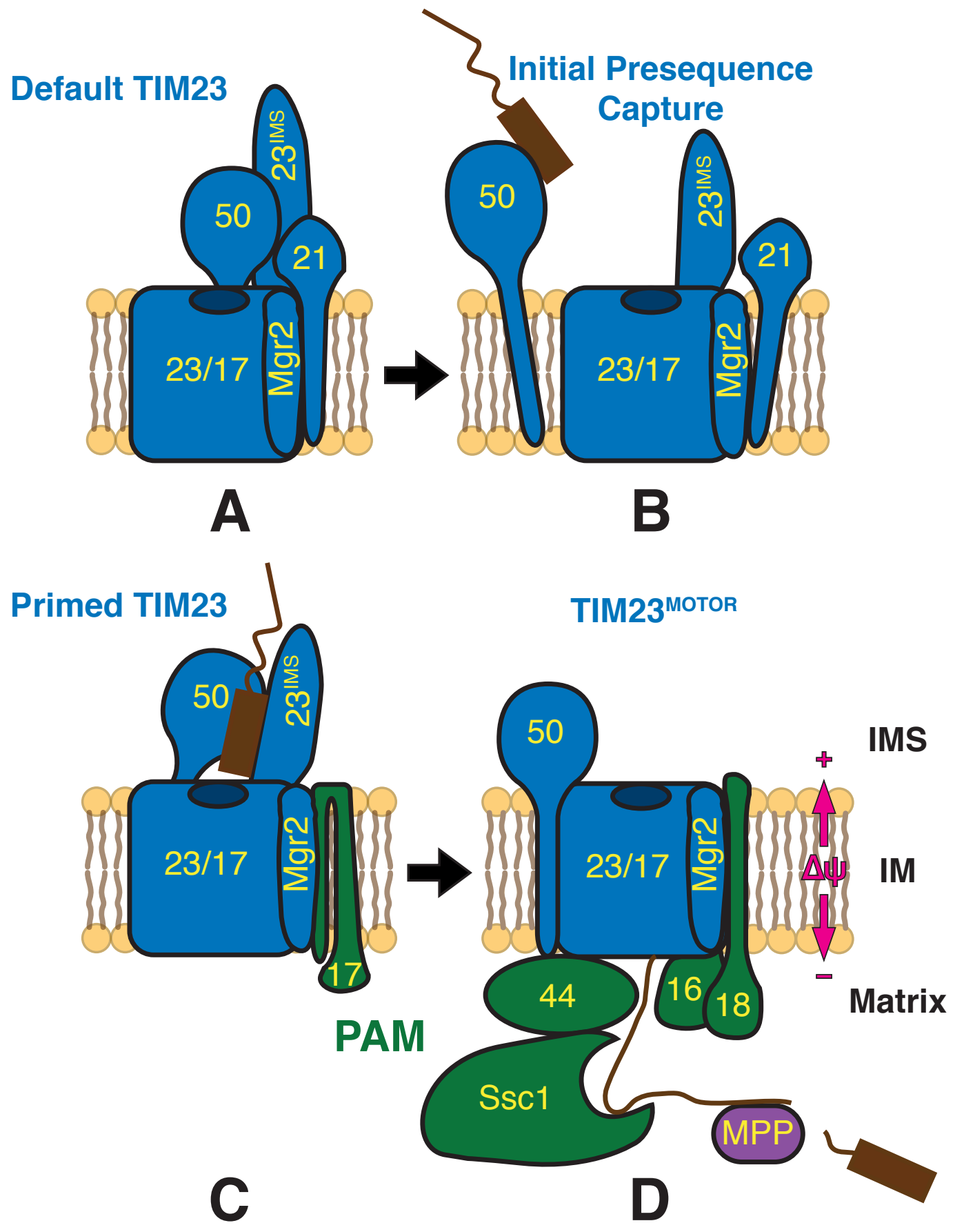

Figure 31. Schematic representation of the dynamic stages of the presequence translocase (TIM23) during matrix import. (A) In the default TIM23 complex Tim50 and Tim21 are in close proximity to each other, held together via the intra membrane space (IMS) domain of Tim23. (B) Tim50, the primary inner membrane (IM) bound presequence receptor initially captures the presequence as it exits the TOM channel at its C-terminal presequence-binding domain. Concomitantly, Tim50 disassociates from Tim21, weakening its association to the presequence translocase. (C) In the primed TIM23 complex, Tim50 associates with the IMS domain of Tim23, 
resulting in presequence hand-off. Pam17, an early acting presequence associated motor (PAM) component, associates with TIM23 leaving the translocase primed for matrix import. (D) The TIM23 $3^{\text {MOTOR }}$ complex is formed with the recruitment of additional PAM subunits, triggering the release of Pam17. The presequence-containing substrate is pulled into the matrix via the ATPdependent Ssc1 (mtHsp70) cycle, with the matrix processing peptidase (MPP) removing the presequence. Figure summarizes the previously published findings (Lytovchenko et al., 2013), and places them within the established translocation model.

\subsection{TIM23 dynamics}

The presequence translocase is one of the most, if not the most, dynamic mitochondrial translocase as it has been described on multiple occasions throughout literature to be present in at least two functionally relevant isoforms: the IM integration competent TIM $23^{\text {SORT }}$ and the matrix translocation competent PAM associated TIM23 ${ }^{\text {MOTOR }}$ form (Chacinska et al., 2005; 2010; Popov-Celeketić et al., 2008a; Saddar et al., 2008; van der Laan et al., 2007). This inherent state of dynamic heterogeneity observed within the presequence translocase greatly adds to its extreme fragility upon membrane solubilization and is likely the single largest hurdle along the path toward high resolution structural data, already available for other OM and IM translocases, namely the TOM and TIM22 complexes (Model et al., 2008; Rehling et al., 2003). The dynamic reorganization is presently believed to be the consequence of the TIM23 complex "reading" and integrating targeting information present within the incoming substrate. This targeting data is present in the form of a presequence with or without a downstream stop-transfer signal. The stop-transfer signal is known to initiate the reorganization of an active translocase (Chacinska et al., 2005; Popov-Celeketić et al., 2008a), allowing for the lateral release of the substrate under translocation into the IM from the TIM $23^{\text {SORT }}$ isoform (van der Laan et al., 2007). The multiple isoform TIM23 model is not without critics (Popov-Celeketić et al., 2008a; Popov-Čeleketić et al., 2011; Tamura et al., 2009), which argue for a single entity model, however, its highly dynamic nature upon substrate presentation is uncontested within both functional TIM23 models. Importantly, publications from groups of both schools of thought have independently confirmed the seemly antagonistic subunit exchange of Tim21 and Pam17 at the active TIM23 complex (Chacinska et al., 2010; Popov-Celeketić et al., 2008a), which is demonstrated here to occur during the early translocase priming stages. However, the dynamic steps undertaken are still not exhaustively characterized and, in particular, the mechanism by which the presequence 
translocase scans the bound substrate for downstream sorting information is currently purely speculative.

Previous works investigating TIM23 dynamics have employed various combinations of supercomplex or translocase isolation, native electrophoresis and various crosslinking techniques (Chacinska et al., 2005; 2010; Popov-Celeketić et al., 2008a; Popov-Čeleketić et al., 2011; Tamura et al., 2009), which suffer from either the lack of a push towards a particular isoform and/or the inability to examine the very early stages of translocase dynamics. Here, complex priming was achieved via presequence peptide incubation, in conjunction with tag-free complex isolation. This combination has allowed for the investigation of very early translocase dynamics in a near-native controlled fashion, monitoring the molecular consequences underlying isoform switching, observed here to involve novel dynamic intra-TIM23 complex subunit interactions.

Controversy amongst current models of TIM23 translocation is rooted in the degree of dynamic association exhibited by the PAM and Tim21 subunits. In this study, great strives were made to clarify these discrepancies between the two prevalent TIM23 models (TIM23 $3^{\text {MOTOR }}$ and TIM23 $3^{\text {SORT }}$ vs. the single entity model), especially in regards to Tim21 dynamics. Here, when, why and how Tim 21 is released from the presequence translocase are presented. When: Tim 21 is released from TIM23 at a very early stage following presequence binding to Tim50. Why: Tim 21 release allows for the subsequent recruitment of Pam17 (Popov-Celeketić et al., 2008a), which assists in ensuing PAM association (Schiller, 2009; van der Laan et al., 2005). How: Following presequence binding to Tim50 at a C-terminal presequence binding domain distant recognition site, Tim 21 dissociates from the TIM23 complex in a Tim23 dependent manner.

Recent studies have laid the framework for the targeted investigation of presequence-induced TIM23 dynamics, as Tim50 is now known to be the an obligatory primary presequence receptor (Marom et al., 2011; Qian et al., 2011; Schulz et al., 2011). Advancing upon previous knowledge, the present study identified a novel presequenceactive dynamic TIM23 interaction between Tim50 and Tim21. This interaction was shown to be mediated by the Tim23 IMS domain and to take place specifically at the presequence translocase and not within a free subunit pool. Importantly, presequences were shown to dissipate the Tim50-Tim21 interaction, resulting in the subsequent release of Tim21 from the TIM23 complex and the concomitant recruitment of Pam17, leaving the presequence translocase primed for further PAM association and ensuing matrix translocation. 
The initial priming events identified in the present study as well as other dynamic events described throughout literature allow for one to mechanistically unravel the presequence translocase, yet key translocation steps are still largely unknown. For example, what are the dynamic subunits allowing for the TIM23 complex to undergo isoform switching during the active translocation of a substrate and which subunits are responsible for the probing of a possible stop-transfer sequence the substrate under translocation? These questions represent only a sliver of the presently mysterious actions of the IM presequence translocase and indentify alluring areas of future investigation. 


\section{Summary and future perspectives}

The present study has investigated the role played by Tom40 during the import of presequence-containing substrates. Using a joint photo-crosslinking mass spectrometry approach, validated within the mitochondrial context (Schulz et al., 2011), the presequence binding sites of Tom 40 were mapped and placed within the recently biochemically validated VDAC based Tom40 homology model (Qiu et al., 2013). Pinpointing the locations of the long hypothesized cis and trans Tom40 presequence interaction sites (Kanamori et al., 1999; Mayer et al., 1995; Rapaport et al., 1998b), a previously identified posttranslationally modified residue (Schmidt et al., 2011), T220, was functionally investigated due to its unambiguous proximity to an identified presequence interaction groove. Through the creation of validated phospho-mimetic point mutants (Gerbeth et al., 2013; Schmidt et al., 2011) the site of presequence interaction was confirmed and functionally linked to the regulation of presequence-mediate OM passage. Moreover, an import conferring relationship was established between the characterized Tom40 presequence binding site and the trans-acting IMS domain of Tom22. The posttansloationally modified Tom 40 presequence recognition site investigated in this study was shown to affect the flux of a matrix-targeted substrate over the entirety of the presequence pathway.

Examining the very next functionally relevant step in presequence translocation, a novel intra-TIM23 subunit interaction was characterized and identified to play a key role in the early stages of TIM23 mediated presequence import. Through the use of a short chemical crosslinking reagent $(2-3 \AA), \mathrm{Cu}^{2+}$, Tim21 was identified as a Tim50 binding partner. The Tim50-Tim21 interaction was recapitulated in an in vitro interaction assay, where Tim23's IMS domain was observed to coordinate the interaction via a Tim23Tim50 interaction dependent mechanism. The Tim50-Tim21 interaction was seen to occur at the presequence translocase and dissociate upon the addition of presequence peptides, triggering in the association of Pam17, leaving the translocase primed for matrix import. Moreover, the presequence peptide mediated priming effect on the TIM23 complex was revealed to occur via presequence binding to a site distant from the established primary IM bound receptor groove within the C-terminus of Tim50 (Schulz et al., 2011). In summary, the stages of presequence initiated TIM23 priming described in this study link presequence presentation in the IMS with the recruitment of the PAM complex, facilitating matrix translocation of the substrate. 
Looking forward to future studies, the present work raises several intriguing questions. As Tom40 has now been confirmed to contain two spatially distinct binding sites, one might assume they act in concert pulling the presequence across the outer membrane, a concept supported by early publications (Mayer et al., 1995; Rapaport et al., 1998b). Utilizing the mapped Tom40 binding sites from the present study, the creation of cis or trans specific mutants would allow for the disentanglement of the individual binding grooves. Moreover, in order for these mutants to be created, the unambiguous assignment of the cis and trans faces of the $\beta$-barrel is obligatory.

Further investigation into the novel, posttranslational substrate regulatory mechanism, through the identification of corresponding Tom40 T220 kinase and phosphatase would enable the in-depth characterization of the OM fine-tuning mechanism. Importantly, the presented mode of substrate kinetic regulation allows for the immediate and direct alteration of mitochondrial substrate flux. Previously publications investigating TOM posttranslational regulation uncovered a mechanism that controls the steady-state levels of the complex through the selective phosphorylation of TOM assembly proteins such as Mim1 and Tom22 (Gerbeth et al., 2013; Schmidt et al., 2011). However, this mechanism is not fast acting due to its dependence on the removal of existing or the creation of de novo TOM complex. It would follow that a fast acting, posttranslational presequence import, regulatory system exists as hinted on in the present study, since a similar Tom70 centered, carrier pathway specific regulatory network has been presented (Schmidt et al., 2011). Moreover, the TOM phosphoproteome study by Schmidt and colleagues has uncovered an explicit IMS localized phosphorylation site within Tom $22^{\mathrm{IMS}}$, T129. It is tempting to connect this posttranslational modification to the Tom40 T220 centered import regulatory system addressed here, yet the exploration of IMS localized kinases and phosphatases is a largely unexplored field with only a small number of soluble perspective members recently identified (Vögtle et al., 2012). These two areas of presequence interaction are presented here to be functionally linked and represent a prime target for future investigations examining the functional consequences to presequence import brought about by Tom40 T220 and Tom22 T129 phosphorylation.

The import mediating substrate interactions of the trans face of the TOM complex is poorly understood to this day. This is highlighted when one compares the rich wealth of publications investigating the cytosolic facing subunits of the TOM complex with the nearly inexistent number examining the functionality of the IMS exposed subunits. In regards to the present findings, two underlying questions arise: (i) what is the nature of the 
observed functional cooperation of Tom 40 and Tom $22^{\text {IMS }}$ at the trans surface of the TOM complex, specifically does sandwiching of the presequence take place similarly to the speculated joint presequence binding by Tom 20 and Tom 22 at the cis face?; (ii) what is the role of IM anchored receptors during late stage TOM translocation, in particular the delineation of the previously observed stabilization function of Tim50 in TOM intermediates (Chacinska et al., 2005)?

At the IM, the TIM23 complex was shown to undergo a series of intra-translocase dynamic interactions, resulting in the matrix import primed presequence translocase. The knowledge gained in the present work would best be supplemented with the investigation of two prominent issues. The first of which is recognized by the restrictions to the priming mechanism presented here, as it is confined to the import of matrix-targeted substrates. This downfall highlights the present void of knowledge regarding the mechanism by which TIM23 scans the substrate under translocation for downstream sorting information and subsequently initiates isoform switching. The second issue is observed when one carefully scrutinizes the late stages of the model presented. Explicitly, how are the dynamics of the PAM subunits linked to the force conveying actions of the motor complex? This issue is presently under intense investigation within the mitochondrial import field (Chacinska et al., 2009; van der Laan et al., 2010), as a functional model of the PAM complex is lacking.

The notion of membrane spanning translocase cooperation is brought front and center when the two topics investigated in the present study are brought together, the functional characterization of late stage presequence-containing substrate TOM translocation and the indiscriminately associated presequence translocase priming steps. This concept has been toyed with since the initial observation of translocation supercomplexes (Schleyer and Neupert, 1985), yet the functional significance of supercomplex formation is still unknown. Expanding upon our understanding of coordinated inner and outer membrane bound receptor interactions will greatly contribute towards a comprehensive understanding of mitochondrial import and lead to the step-bystep mechanistic delineation of presequence mediated import. 


\section{References}

Abe, Y., Shodai, T., Muto, T., Mihara, K., Torii, H., Nishikawa, S., Endo, T., and Kohda, D. (2000). Structural basis of presequence recognition by the mitochondrial protein import receptor Tom20. Cell 100, 551-560.

Ahting, U., Thieffry, M., Engelhardt, H., Hegerl, R., Neupert, W., and Nussberger, S. (2001). Tom40, the pore-forming component of the protein-conducting TOM channel in the outer membrane of mitochondria. J. Cell Biol. 153, 1151-1160.

Ahting, U., Thun, C., Hegerl, R., Typke, D., Nargang, F.E., Neupert, W., and Nussberger, S. (1999). The TOM core complex: the general protein import pore of the outer membrane of mitochondria. J. Cell Biol. 147, 959-968.

Albrecht, R., Rehling, P., Chacinska, A., Brix, J., Cadamuro, S.A., Volkmer, R., Guiard, B., Pfanner, N., and Zeth, K. (2006). The Tim 21 binding domain connects the preprotein translocases of both mitochondrial membranes. EMBO Rep. 7, 1233-1238.

Albuquerque, C.P., Smolka, M.B., Payne, S.H., Bafna, V., Eng, J., and Zhou, H. (2008). A multidimensional chromatography technology for in-depth phosphoproteome analysis. Mol. Cell Proteomics 7, 1389-1396.

Alconada, A., Kübrich, M., Moczko, M., Hönlinger, A., and Pfanner, N. (1995). The mitochondrial receptor complex: the small subunit Mom8b/Isp6 supports association of receptors with the general insertion pore and transfer of preproteins. Mol. Cell. Biol. 15, 6196-6205.

Alder, N.N., Sutherland, J., Buhring, A.I., Jensen, R.E., and Johnson, A.E. (2008). Quaternary structure of the mitochondrial TIM23 complex reveals dynamic association between Tim23p and other subunits. Mol. Biol. Cell 19, 159-170.

Allen, S., Balabanidou, V., Sideris, D.P., Lisowsky, T., and Tokatlidis, K. (2005). Erv1 mediates the Mia40-dependent protein import pathway and provides a functional link to the respiratory chain by shuttling electrons to cytochrome c. J. Mol. Biol. 353, 937-944.

Allison, D.S., and Schatz, G. (1986). Artificial mitochondrial presequences. Proc. Natl. Acad. Sci. USA 83, 9011-9015.

Althoff, T., Mills, D.J., Popot, J.-L., and Kühlbrandt, W. (2011). Arrangement of electron transport chain components in bovine mitochondrial supercomplex I1III2IV1. EMBO J. $30,4652-4664$.

Andersson, G.E., Karlberg, O., Canback, B., and Kurland, C.G. (2003). On the origin of mitochondria: a genomics perspective. Philos. Trans. R. Soc. Lond., B, Biol. Sci. 358, $165-179$.

Asai, T., Takahashi, T., Esaki, M., Nishikawa, S.-I., Ohtsuka, K., Nakai, M., and Endo, T. (2004). Reinvestigation of the requirement of cytosolic ATP for mitochondrial protein import. J. Biol. Chem. 279, 19464-19470. 
Baker, K.P., Schaniel, A., Vestweber, D., and Schatz, G. (1990). A yeast mitochondrial outer membrane protein essential for protein import and cell viability. Nature 348, 605609.

Banci, L., Bertini, I., Calderone, V., Cefaro, C., Ciofi-Baffoni, S., Gallo, A., Kallergi, E., Lionaki, E., Pozidis, C., and Tokatlidis, K. (2011). Molecular recognition and substrate mimicry drive the electron-transfer process between MIA40 and ALR. Proc. Natl. Acad. Sci. USA $108,4811-4816$.

Banci, L., Bertini, I., Cefaro, C., Ciofi-Baffoni, S., Gallo, A., Martinelli, M., Sideris, D.P., Katrakili, N., and Tokatlidis, K. (2009). MIA40 is an oxidoreductase that catalyzes oxidative protein folding in mitochondria. Nat. Struct. Mol. Biol. 16, 198-206.

Barrientos, A., and Ugalde, C. (2013). I function, therefore I am: overcoming skepticism about mitochondrial supercomplexes. Cell Metab. 18, 147-149.

Bauer, M.F., Sirrenberg, C., Neupert, W., and Brunner, M. (1996). Role of Tim23 as voltage sensor and presequence receptor in protein import into mitochondria. Cell 87, 3341.

Becker, L., Bannwarth, M., Meisinger, C., Hill, K., Model, K., Krimmer, T., Casadio, R., Truscott, K.N., Schulz, G.E., Pfanner, N., et al. (2005). Preprotein translocase of the outer mitochondrial membrane: reconstituted Tom40 forms a characteristic TOM pore. J. Mol. Biol. 353, 1011-1020.

Becker, T., Wenz, L.S., Kruger, V., Lehmann, W., Muller, J.M., Goroncy, L., Zufall, N., Lithgow, T., Guiard, B., Chacinska, A., et al. (2011). The mitochondrial import protein Mim1 promotes biogenesis of multispanning outer membrane proteins. J. Cell Biol. 194, 387-395.

Becker, T., Guiard, B., Thornton, N., Zufall, N., Stroud, D.A., Wiedemann, N., and Pfanner, N. (2010). Assembly of the mitochondrial protein import channel: role of Tom5 in two-stage interaction of Tom40 with the SAM complex. Mol. Biol. Cell. 21, 3106-3113.

Becker, T., Pfannschmidt, S., Guiard, B., Stojanovski, D., Milenkovic, D., Kutik, S., Pfanner, N., Meisinger, C., and Wiedemann, N. (2008). Biogenesis of the mitochondrial TOM complex: Mim1 promotes insertion and assembly of signal-anchored receptors. J. Biol. Chem. 283, 120-127.

Beverly, K.N., Sawaya, M.R., Schmid, E., and Koehler, C.M. (2008). The Tim8-Tim13 complex has multiple substrate binding sites and binds cooperatively to Tim23. J. Mol. Biol. 382, 1144-1156.

Bhattacharya, D., Archibald, J.M., Weber, A.P.M., and Reyes-Prieto, A. (2007). How do endosymbionts become organelles? Understanding early events in plastid evolution. Bioessays 29, 1239-1246.

Bien, M., Longen, S., Wagener, N., Chwalla, I., Herrmann, J.M., and Riemer, J. (2010). Mitochondrial disulfide bond formation is driven by intersubunit electron transfer in Erv1 and proofread by glutathione. Mol. Cell 37, 516-528. 
Bihlmaier, K., Mesecke, N., Terziyska, N., Bien, M., Hell, K., and Herrmann, J.M. (2007). The disulfide relay system of mitochondria is connected to the respiratory chain. J. Cell Biol. 179, 389-395.

Bohnert, M., Rehling, P., Guiard, B., Herrmann, J.M., Pfanner, N., and van der Laan, M. (2010). Cooperation of stop-transfer and conservative sorting mechanisms in mitochondrial protein transport. Curr. Biol. 20, 1227-1232.

Bonneaud, N., Ozier-Kalogeropoulos, O., Li, G.Y., Labouesse, M., Minvielle-Sebastia, L., and Lacroute, F. (1991). A family of low and high copy replicative, integrative and singlestranded S. cerevisiae/E. coli shuttle vectors. Yeast 7, 609-615.

Bos, M.P., Robert, V., and Tommassen, J. (2007). Functioning of outer membrane protein assembly factor Omp85 requires a single POTRA domain. EMBO Rep. 8, 1149-1154.

Bömer, U., Meijer, M., Guiard, B., Dietmeier, K., Pfanner, N., and Rassow, J. (1997). The sorting route of cytochrome b2 branches from the general mitochondrial import pathway at the preprotein translocase of the inner membrane. J. Biol. Chem. 272, 30439-30446.

Brix, J., Dietmeier, K., and Pfanner, N. (1997). Differential recognition of preproteins by the purified cytosolic domains of the mitochondrial import receptors Tom20, Tom22, and Tom70. J. Biol. Chem. 272, 20730-20735.

Brix, J., Rüdiger, S., Bukau, B., Schneider-Mergener, J., and Pfanner, N. (1999).

Distribution of binding sequences for the mitochondrial import receptors Tom20, Tom22, and Tom70 in a presequence-carrying preprotein and a non-cleavable preprotein. J. Biol. Chem. 274, 16522-16530.

Brix, J., Ziegler, G.A., Dietmeier, K., Schneider-Mergener, J., Schulz, G.E., and Pfanner, N. (2000). The mitochondrial import receptor Tom70: identification of a $25 \mathrm{kDa}$ core domain with a specific binding site for preproteins. J. Mol. Biol. 303, 479-488.

Cavalier-Smith, T. (2006). Origin of mitochondria by intracellular enslavement of a photosynthetic purple bacterium. P. Roy. Soc. B-Biol. Sci. 273, 1943-1952.

Chacinska, A., Koehler, C.M., Milenkovic, D., Lithgow, T., and Pfanner, N. (2009). Importing mitochondrial proteins: machineries and mechanisms. Cell 138, 628-644.

Chacinska, A., Lind, M., Frazier, A.E., Dudek, J., Meisinger, C., Geissler, A., Sickmann, A., Meyer, H.E., Truscott, K.N., Guiard, B., et al. (2005). Mitochondrial presequence translocase: switching between TOM tethering and motor recruitment involves Tim 21 and Tim17. Cell 120, 817-829.

Chacinska, A., Pfannschmidt, S., Wiedemann, N., Kozjak, V., Sanjuán Szklarz, L.K., Schulze-Specking, A., Truscott, K.N., Guiard, B., Meisinger, C., and Pfanner, N. (2004). Essential role of Mia40 in import and assembly of mitochondrial intermembrane space proteins. EMBO J. 23, 3735-3746.

Chacinska, A., Rehling, P., Guiard, B., Frazier, A.E., Schulze-Specking, A., Pfanner, N., Voos, W., and Meisinger, C. (2003). Mitochondrial translocation contact sites: separation of dynamic and stabilizing elements in formation of a TOM-TIM-preprotein

supercomplex. EMBO J. 22, 5370-5381. 
Chacinska, A., van der Laan, M., Mehnert, C.S., Guiard, B., Mick, D.U., Hutu, D.P., Truscott, K.N., Wiedemann, N., Meisinger, C., Pfanner, N., et al. (2010). Distinct forms of mitochondrial TOM-TIM supercomplexes define signal-dependent states of preprotein sorting. Mol. Cell. Biol. 30, 307-318.

Chan, N.C., and Lithgow, T. (2008). The peripheral membrane subunits of the SAM complex function codependently in mitochondrial outer membrane biogenesis. Mol. Biol. Cell 19, 126-136.

Chi, A., Huttenhower, C., Geer, L.Y., Coon, J.J., Syka, J.E.P., Bai, D.L., Shabanowitz, J., Burke, D.J., Troyanskaya, O.G., and Hunt, D.F. (2007). Analysis of phosphorylation sites on proteins from Saccharomyces cerevisiae by electron transfer dissociation (ETD) mass spectrometry. Proc. Natl. Acad. Sci. USA 104, 2193-2198.

Cruciat, C.M., Brunner, S., Baumann, F., Neupert, W., and Stuart, R.A. (2000). The cytochrome $\mathrm{bc} 1$ and cytochrome $\mathrm{c}$ oxidase complexes associate to form a single supracomplex in yeast mitochondria. J. Biol. Chem. 275, 18093-18098.

Curran, S.P., Leuenberger, D., Oppliger, W., and Koehler, C.M. (2002). The Tim9pTim10p complex binds to the transmembrane domains of the ADP/ATP carrier. EMBO J. 21,942-953.

D'Silva, P.D., Schilke, B., Walter, W., Andrew, A., and Craig, E.A. (2003). J protein cochaperone of the mitochondrial inner membrane required for protein import into the mitochondrial matrix. Proc. Natl. Acad. Sci. USA 100, 13839-13844.

D'Silva, P.R., Schilke, B., Hayashi, M., and Craig, E.A. (2008). Interaction of the J-protein heterodimer Pam18/Pam16 of the mitochondrial import motor with the translocon of the inner membrane. Mol. Biol. Cell 19, 424-432.

D'Silva, P.R., Schilke, B., Walter, W., and Craig, E.A. (2005). Role of Pam16's degenerate $\mathrm{J}$ domain in protein import across the mitochondrial inner membrane. Proc. Natl. Acad. Sci. USA 102, 12419-12424.

Dabir, D.V., Leverich, E.P., Kim, S.-K., Tsai, F.D., Hirasawa, M., Knaff, D.B., and Koehler, C.M. (2007). A role for cytochrome c and cytochrome c peroxidase in electron shuttling from Erv1. EMBO J. 26, 4801-4811.

Davis, A.J., Alder, N.N., Jensen, R.E., and Johnson, A.E. (2007). The Tim9p/10p and Tim8p/13p complexes bind to specific sites on Tim23p during mitochondrial protein import. Mol. Biol. Cell 18, 475-486.

de Duve, C. (2007). The origin of eukaryotes: a reappraisal. Nat. Rev. Genet. 8, 395-403.

Dekker, P.J., Martin, F., Maarse, A.C., Bömer, U., Müller, H., Guiard, B., Meijer, M., Rassow, J., and Pfanner, N. (1997). The Tim core complex defines the number of mitochondrial translocation contact sites and can hold arrested preproteins in the absence of matrix Hsp70-Tim44. EMBO J. 16, 5408-5419.

Dekker, P.J., Ryan, M.T., Brix, J., Müller, H., Hönlinger, A., and Pfanner, N. (1998). Preprotein translocase of the outer mitochondrial membrane: molecular dissection and assembly of the general import pore complex. Mol. Cell. Biol. 18, 6515-6524. 
Dembowski, M., Künkele, K.P., Nargang, F.E., Neupert, W., and Rapaport, D. (2001). Assembly of Tom6 and Tom7 into the TOM core complex of Neurospora crassa. J. Biol. Chem. 276, 17679-17685.

Dietmeier, K., Hönlinger, A., Bömer, U., Dekker, P.J., Eckerskorn, C., Lottspeich, F., Kübrich, M., and Pfanner, N. (1997). Tom5 functionally links mitochondrial preprotein receptors to the general import pore. Nature 388, 195-200.

Dolezal, P., Likic, V., Tachezy, J., and Lithgow, T. (2006). Evolution of the molecular machines for protein import into mitochondria. Science 313, 314-318.

Dormán, G., and Prestwich, G.D. (1994). Benzophenone photophores in biochemistry. Biochemistry 33, 5661-5673.

Dudek, J., Rehling, P., and van der Laan, M. (2013). Mitochondrial protein import: common principles and physiological networks. Biochim. Biophys. Acta 1833, 274-285.

Dudkina, N.V., Kudryashev, M., Stahlberg, H., and Boekema, E.J. (2011). Interaction of complexes I, III, and IV within the bovine respirasome by single particle cryoelectron tomography. Proc. Natl. Acad. Sci. USA 108, 15196-15200.

Dyall, S.D. (2004). Ancient Invasions: From Endosymbionts to Organelles. Science 304, 253-257.

Endo, T., and Yamano, K. (2010). Transport of proteins across or into the mitochondrial outer membrane. Biochim. Biophys. Acta 1803, 706-714.

Endres, M., Neupert, W., and Brunner, M. (1999). Transport of the ADP/ATP carrier of mitochondria from the TOM complex to the TIM22.54 complex. EMBO J. 18, 3214-3221.

Esaki, M., Kanamori, T., Nishikawa, S.I., Shin, I., Schultz, P.G., and Endo, T. (2003). Tom40 protein import channel binds to non-native proteins and prevents their aggregation. Nat. Struct. Biol. 10, 988-994.

Frazier, A.E., Chacinska, A., Truscott, K.N., Guiard, B., Pfanner, N., and Rehling, P. (2003). Mitochondria use different mechanisms for transport of multispanning membrane proteins through the intermembrane space. Mol. Cell. Biol. 23, 7818-7828.

Frazier, A.E., Dudek, J., Guiard, B., Voos, W., Li, Y., Lind, M., Meisinger, C., Geissler, A., Sickmann, A., Meyer, H.E., et al. (2004). Pam16 has an essential role in the mitochondrial protein import motor. Nat. Struct. Mol. Biol. 11, 226-233.

Gabaldón, T., and Huynen, M.A. (2003). Reconstruction of the proto-mitochondrial metabolism. Science 301, 609.

Gabriel, K., Egan, B., and Lithgow, T. (2003). Tom40, the import channel of the mitochondrial outer membrane, plays an active role in sorting imported proteins. EMBO J. $22,2380-2386$.

Gabriel, K., Milenkovic, D., Chacinska, A., Müller, J., Guiard, B., Pfanner, N., and Meisinger, C. (2007). Novel mitochondrial intermembrane space proteins as substrates of the MIA import pathway. J. Mol. Biol. 365, 612-620. 
Gaikwad, A.S., and Cumsky, M.G. (1994). The use of chemical cross-linking to identify proteins that interact with a mitochondrial presequence. J. Biol. Chem. 269, 6437-6443.

Gambill, B.D., Voos, W., Kang, P.J., Miao, B., Langer, T., Craig, E.A., and Pfanner, N. (1993). A dual role for mitochondrial heat shock protein 70 in membrane translocation of preproteins. J. Cell Biol. 123, 109-117.

Gebert, M., Schrempp, S.G., Mehnert, C.S., Heißwolf, A.K., Oeljeklaus, S., Ieva, R., Bohnert, M., Malsburg, von der, K., Wiese, S., Kleinschroth, T., et al. (2012). Mgr2 promotes coupling of the mitochondrial presequence translocase to partner complexes. J. Cell Biol. 197, 595-604.

Gebert, N., Chacinska, A., Wagner, K., Guiard, B., Koehler, C.M., Rehling, P., Pfanner, N., and Wiedemann, N. (2008). Assembly of the three small Tim proteins precedes docking to the mitochondrial carrier translocase. EMBO Rep. 9, 548-554.

Gebert, N., Gebert, M., Oeljeklaus, S., Malsburg, von der, K., Stroud, D.A., Kulawiak, B., Wirth, C., Zahedi, R.P., Dolezal, P., Wiese, S., et al. (2011). Dual function of Sdh3 in the respiratory chain and TIM22 protein translocase of the mitochondrial inner membrane. Mol. Cell 44, 811-818.

Gerbeth, C., Schmidt, O., Rao, S., Harbauer, A.B., Mikropoulou, D., Opalińska, M., Guiard, B., Pfanner, N., and Meisinger, C. (2013). Glucose-induced regulation of protein import receptor Tom 22 by cytosolic and mitochondria-bound kinases. Cell Metab. 18, $578-587$.

Gessmann, D., Flinner, N., Pfannstiel, J., Schlösinger, A., Schleiff, E., Nussberger, S., and Mirus, O. (2011). Structural elements of the mitochondrial preprotein-conducting channel Tom40 dissolved by bioinformatics and mass spectrometry. Biochim. Biophys. Acta 1807, 1647-1657.

Gevorkyan-Airapetov, L., Zohary, K., Popov-Celeketić, D., Mapa, K., Hell, K., Neupert, W., Azem, A., and Mokranjac, D. (2009). Interaction of Tim23 with Tim50 Is essential for protein translocation by the mitochondrial TIM23 complex. J. Biol. Chem. 284, 48654872 .

Gietz, R.D., and Schiestl, R.H. (2007). High-efficiency yeast transformation using the LiAc/SS carrier DNA/PEG method. Nat. Protoc. 2, 31-34.

Glick, B.S., Brandt, A., Cunningham, K., Müller, S., Hallberg, R.L., and Schatz, G. (1992). Cytochromes $\mathrm{c} 1$ and $\mathrm{b} 2$ are sorted to the intermembrane space of yeast mitochondria by a stop-transfer mechanism. Cell 69, 809-822.

Gnad, F., de Godoy, L.M.F., Cox, J., Neuhauser, N., Ren, S., Olsen, J.V., and Mann, M. (2009). High-accuracy identification and bioinformatic analysis of in vivo protein phosphorylation sites in yeast. Proteomics 9, 4642-4652.

Gordon, D.M., Wang, J., Amutha, B., and Pain, D. (2001). Self-association and precursor protein binding of Saccharomyces cerevisiae Tom40p, the core component of the protein translocation channel of the mitochondrial outer membrane. Biochem. J. 356, 207-215. 
Gray, M.W., Burger, G., and Lang, B.F. (1999). Mitochondrial evolution. Science 283, $1476-1481$.

Gross, D.P., Burgard, C.A., Reddehase, S., Leitch, J.M., Culotta, V.C., and Hell, K. (2011). Mitochondrial Ccs 1 contains a structural disulfide bond crucial for the import of this unconventional substrate by the disulfide relay system. Mol. Biol. Cell 22, 3758-3767.

Gross, J., and Bhattacharya, D. (2009). Mitochondrial and plastid evolution in eukaryotes: an outsiders' perspective. Nat. Rev. Genet. 10, 495-505.

Grumbt, B., Stroobant, V., Terziyska, N., Israel, L., and Hell, K. (2007). Functional characterization of Mia40p, the central component of the disulfide relay system of the mitochondrial intermembrane space. J. Biol. Chem. 282, 37461-37470.

Habib, S.J., Waizenegger, T., Niewienda, A., Paschen, S.A., Neupert, W., and Rapaport, D. (2007). The N-terminal domain of Tob55 has a receptor-like function in the biogenesis of mitochondrial beta-barrel proteins. J. Cell Biol. 176, 77-88.

Hackenbrock, C.R., Chazotte, B., and Gupte, S.S. (1986). The random collision model and a critical assessment of diffusion and collision in mitochondrial electron transport. J. Bioenerg. Biomembr. 18, 331-368.

Harner, M., Neupert, W., and Deponte, M. (2011). Lateral release of proteins from the TOM complex into the outer membrane of mitochondria. EMBO J. 30, 3232-3241.

Hatefi, Y. (1985). The mitochondrial electron transport and oxidative phosphorylation system. Annu. Rev. Biochem. 54, 1015-1069.

Hill, K., Model, K., Ryan, M.T., Dietmeier, K., Martin, F., Wagner, R., and Pfanner, N. (1998). Tom40 forms the hydrophilic channel of the mitochondrial import pore for preproteins. Nature 395, 516-521.

Holt, L.J., Tuch, B.B., Villén, J., Johnson, A.D., Gygi, S.P., and Morgan, D.O. (2009). Global analysis of Cdk1 substrate phosphorylation sites provides insights into evolution. Science $325,1682-1686$.

Hoppins, S.C., and Nargang, F.E. (2004). The Tim8-Tim13 complex of Neurospora crassa functions in the assembly of proteins into both mitochondrial membranes. J. Biol. Chem. $279,12396-12405$.

Hönlinger, A., Bömer, U., Alconada, A., Eckerskorn, C., Lottspeich, F., Dietmeier, K., and Pfanner, N. (1996). Tom7 modulates the dynamics of the mitochondrial outer membrane translocase and plays a pathway-related role in protein import. EMBO J. 15, 2125-2137.

Hulett, J.M., Lueder, F., Chan, N.C., Perry, A.J., Wolynec, P., Likic, V.A., Gooley, P.R., and Lithgow, T. (2008). The transmembrane segment of Tom 20 is recognized by Mim1 for docking to the mitochondrial TOM complex. J. Mol. Biol. 376, 694-704.

Hwang, D.K., Claypool, S.M., Leuenberger, D., Tienson, H.L., and Koehler, C.M. (2007). Tim $54 p$ connects inner membrane assembly and proteolytic pathways in the mitochondrion. J. Cell Biol. 178, 1161-1175. 
Imai, K., Gromiha, M.M., and Horton, P. (2008). Mitochondrial beta-barrel proteins, an exclusive club? Cell 135, 1158-9-authorreply1159-60.

Ishikawa, D., Yamamoto, H., Tamura, Y., Moritoh, K., and Endo, T. (2004). Two novel proteins in the mitochondrial outer membrane mediate beta-barrel protein assembly. J. Cell Biol. 166, 621-627.

Jahn, O., Eckart, K., Brauns, O., Tezval, H., and Spiess, J. (2002). The binding protein of corticotropin-releasing factor: ligand-binding site and subunit structure. Proc. Natl. Acad. Sci. USA 99, 12055-12060.

Janke, C., Magiera, M.M., Rathfelder, N., Taxis, C., Reber, S., Maekawa, H., MorenoBorchart, A., Doenges, G., Schwob, E., Schiebel, E., et al. (2004). A versatile toolbox for PCR-based tagging of yeast genes: new fluorescent proteins, more markers and promoter substitution cassettes. Yeast 1-16.

Juin, P., Thieffry, M., Henry, J.P., and Vallette, F.M. (1997). Relationship between the peptide-sensitive channel and the mitochondrial outer membrane protein translocation machinery. J. Biol. Chem. 272, 6044-6050.

Kanamori, T., Nishikawa, S., Nakai, M., Shin, I., Schultz, P.G., and Endo, T. (1999). Uncoupling of transfer of the presequence and unfolding of the mature domain in precursor translocation across the mitochondrial outer membrane. Proc. Natl. Acad. Sci. USA 96, 3634-3639.

Kang, P.J., Ostermann, J., Shilling, J., Neupert, W., Craig, E.A., and Pfanner, N. (1990). Requirement for hsp70 in the mitochondrial matrix for translocation and folding of precursor proteins. Nature 348, 137-143.

Kassenbrock, C.K., Cao, W., and Douglas, M.G. (1993). Genetic and biochemical characterization of ISP6, a small mitochondrial outer membrane protein associated with the protein translocation complex. EMBO J. 12, 3023-3034.

Kato, H., and Mihara, K. (2008). Identification of Tom5 and Tom6 in the preprotein translocase complex of human mitochondrial outer membrane. Biochem. Biophys. Res. Commun. 369, 958-963.

Kawamata, H., and Manfredi, G. (2010). Import, maturation, and function of SOD1 and its copper chaperone CCS in the mitochondrial intermembrane space. Antioxid. Redox Signal. $13,1375-1384$.

Kawano, S., Yamano, K., Naoé, M., Momose, T., Terao, K., Nishikawa, S.I., Watanabe, N., and Endo, T. (2009). Structural basis of yeast Tim40/Mia40 as an oxidative translocator in the mitochondrial intermembrane space. Proc. Natl. Acad. Sci. USA 106, 14403-14407.

Kearse, M., Moir, R., Wilson, A., Stones-Havas, S., Cheung, M., Sturrock, S., Buxton, S., Cooper, A., Markowitz, S., Duran, C., et al. (2012). Geneious Basic: an integrated and extendable desktop software platform for the organization and analysis of sequence data. Bioinformatics 28, 1647-1649. 
Kemper, C., Habib, S.J., Engl, G., Heckmeyer, P., Dimmer, K.S., and Rapaport, D. (2008). Integration of tail-anchored proteins into the mitochondrial outer membrane does not require any known import components. J. Cell Sci. 121, 1990-1998.

Klöppel, C., Suzuki, Y., Kojer, K., Petrungaro, C., Longen, S., Fiedler, S., Keller, S., and Riemer, J. (2011). Mia40-dependent oxidation of cysteines in domain I of Ccs1 controls its distribution between mitochondria and the cytosol. Mol. Biol. Cell 22, 3749-3757.

Koehler, C.M., Merchant, S., Oppliger, W., Schmid, K., Jarosch, E., Dolfini, L., Junne, T., Schatz, G., and Tokatlidis, K. (1998). Tim9p, an essential partner subunit of Tim10p for the import of mitochondrial carrier proteins. EMBO J. 17, 6477-6486.

Koll, H., Guiard, B., Rassow, J., Ostermann, J., Horwich, A.L., Neupert, W., and Hartl, F.U. (1992). Antifolding activity of hsp60 couples protein import into the mitochondrial matrix with export to the intermembrane space. Cell 68, 1163-1175.

Komiya, T., Rospert, S., Koehler, C., Looser, R., Schatz, G., and Mihara, K. (1998). Interaction of mitochondrial targeting signals with acidic receptor domains along the protein import pathway: evidence for the "acid chain" hypothesis. EMBO J. 17, 38863898.

Kornmann, B., Currie, E., Collins, S.R., Schuldiner, M., Nunnari, J., Weissman, J.S., and Walter, P. (2009). An ER-mitochondria tethering complex revealed by a synthetic biology screen. Science 325, 477-481.

Kovermann, P., Truscott, K.N., Guiard, B., Rehling, P., Sepuri, N.B., Müller, H., Jensen, R.E., Wagner, R., and Pfanner, N. (2002). Tim22, the essential core of the mitochondrial protein insertion complex, forms a voltage-activated and signal-gated channel. Mol. Cell 9 , $363-373$.

Kozany, C., Mokranjac, D., Sichting, M., Neupert, W., and Hell, K. (2004). The J domainrelated cochaperone Tim16 is a constituent of the mitochondrial TIM23 preprotein translocase. Nat. Struct. Mol. Biol. 11, 234-241.

Krayl, M., Lim, J.H., Martin, F., Guiard, B., and Voos, W. (2007). A cooperative action of the ATP-dependent import motor complex and the inner membrane potential drives mitochondrial preprotein import. Mol. Cell. Biol. 27, 411-425.

Kurland, C.G., and Andersson, S.G.E. (2000). Origin and Evolution of the Mitochondrial Proteome. Microbiol. Mol. Biol. R. 64, 786-820.

Kutik, S., Stojanovski, D., Becker, L., Becker, T., Meinecke, M., Krüger, V., Prinz, C., Meisinger, C., Guiard, B., Wagner, R., et al. (2008). Dissecting Membrane Insertion of Mitochondrial $\beta$-Barrel Proteins. Cell 132, 1011-1024.

Künkele, K.P., Juin, P., Pompa, C., Nargang, F.E., Henry, J.P., Neupert, W., Lill, R., and Thieffry, M. (1998). The isolated complex of the translocase of the outer membrane of mitochondria. Characterization of the cation-selective and voltage-gated preproteinconducting pore. J. Biol. Chem. 273, 31032-31039.

Laemmli, U.K. (1970). Cleavage of structural proteins during the assembly of the head of bacteriophage T4. Nature 227, 680-685. 
Lapuente-Brun, E., Moreno-Loshuertos, R., Acin-Perez, R., Latorre-Pellicer, A., Colás, C., Balsa, E., Perales-Clemente, E., Quirós, P.M., Calvo, E., Rodriguez-Hernandez, M.A., et al. (2013). Supercomplex assembly determines electron flux in the mitochondrial electron transport chain. Science 340, 1567-1570.

Li, X., Gerber, S.A., Rudner, A.D., Beausoleil, S.A., Haas, W., Villén, J., Elias, J.E., and Gygi, S.P. (2007). Large-scale phosphorylation analysis of alpha-factor-arrested Saccharomyces cerevisiae. J. Proteome Res. 6, 1190-1197.

Li, Y., Dudek, J., Guiard, B., Pfanner, N., Rehling, P., and Voos, W. (2004). The presequence translocase-associated protein import motor of mitochondria. Pam 16 functions in an antagonistic manner to Pam18. J. Biol. Chem. 279, 38047-38054.

Lipinski, K.A., Kaniak-Golik, A., and Golik, P. (2010). Maintenance and expression of the S. cerevisiae mitochondrial genome--from genetics to evolution and systems biology. Biochim. Biophys. Acta 1797, 1086-1098.

Liu, Q., D'Silva, P., Walter, W., Marszalek, J., and Craig, E.A. (2003). Regulated cycling of mitochondrial Hsp70 at the protein import channel. Science 300, 139-141.

Luciano, P., Vial, S., Vergnolle, M.A., Dyall, S.D., Robinson, D.R., and Tokatlidis, K. (2001). Functional reconstitution of the import of the yeast ADP/ATP carrier mediated by the TIM10 complex. EMBO J. 20, 4099-4106.

Lueder, F., and Lithgow, T. (2009). The three domains of the mitochondrial outer membrane protein Mim1 have discrete functions in assembly of the TOM complex. FEBS Lett. 583, 1475-1480.

Lytovchenko, O., Melin, J., Schulz, C., Kilisch, M., Hutu, D.P., and Rehling, P. (2013). Signal recognition initiates reorganization of the presequence translocase during protein import. EMBO J. 32, 886-898.

Mahendran, K.R., Romero-Ruiz, M., Schlösinger, A., Winterhalter, M., and Nussberger, S. (2012). Protein Translocation through Tom40: Kinetics of Peptide Release. Biophys. J. $102,39-47$.

Malsburg, von der, K., Müller, J.M., Bohnert, M., Oeljeklaus, S., Kwiatkowska, P., Becker, T., Loniewska-Lwowska, A., Wiese, S., Rao, S., Milenkovic, D., et al. (2011). Dual role of mitofilin in mitochondrial membrane organization and protein biogenesis. Dev. Cell 21, 694-707.

Marom, M., Dayan, D., Demishtein-Zohary, K., Mokranjac, D., Neupert, W., and Azem, A. (2011). Direct Interaction of Mitochondrial Targeting Presequences with Purified Components of the TIM23 Protein Complex. J. Biol. Chem. 286, 43809-43815.

Martin, J., Mahlke, K., and Pfanner, N. (1991). Role of an energized inner membrane in mitochondrial protein import. Delta psi drives the movement of presequences. J. Biol. Chem. 266, 18051-18057.

Martinez-Caballero, S., Grigoriev, S.M., Herrmann, J.M., Campo, M.L., and Kinnally, K.W. (2007). Tim $17 p$ regulates the twin pore structure and voltage gating of the mitochondrial protein import complex TIM23. J. Biol. Chem. 282, 3584-3593. 
Mayer, A., Neupert, W., and Lill, R. (1995). Mitochondrial protein import: reversible binding of the presequence at the trans side of the outer membrane drives partial translocation and unfolding. Cell 80, 127-137.

Meier, S., Neupert, W., and Herrmann, J.M. (2005). Conserved N-terminal negative charges in the Tim17 subunit of the TIM23 translocase play a critical role in the import of preproteins into mitochondria. J. Biol. Chem. 280, 7777-7785.

Meinecke, M., Wagner, R., Kovermann, P., Guiard, B., Mick, D.U., Hutu, D.P., Voos, W., Truscott, K.N., Chacinska, A., Pfanner, N., et al. (2006). Tim50 maintains the permeability barrier of the mitochondrial inner membrane. Science 312, 1523-1526.

Meisinger, C., Ryan, M.T., Hill, K., Model, K., Lim, J.H., Sickmann, A., Müller, H., Meyer, H.E., Wagner, R., and Pfanner, N. (2001). Protein import channel of the outer mitochondrial membrane: a highly stable Tom40-Tom 22 core structure differentially interacts with preproteins, small tom proteins, and import receptors. Mol. Cell. Biol. 21, 2337-2348.

Meisinger, C., Pfanner, N., and Truscott, K.N. (2006). Isolation of yeast mitochondria. Methods Mol. Biol. 313, 33-39.

Meisinger, C., Rissler, M., Chacinska, A., Szklarz, L.K.S., Milenkovic, D., Kozjak, V., Schönfisch, B., Lohaus, C., Meyer, H.E., Yaffe, M.P., et al. (2004). The mitochondrial morphology protein Mdm10 functions in assembly of the preprotein translocase of the outer membrane. Dev. Cell 7, 61-71.

Mesecke, N., Terziyska, N., Kozany, C., Baumann, F., Neupert, W., Hell, K., and Herrmann, J.M. (2005). A disulfide relay system in the intermembrane space of mitochondria that mediates protein import. Cell 121, 1059-1069.

Miao, B., Davis, J.E., and Craig, E.A. (1997). Mge1 functions as a nucleotide release factor for Ssc1, a mitochondrial Hsp70 of Saccharomyces cerevisiae. J. Mol. Biol. 265, $541-552$.

Milenkovic, D., Gabriel, K., Guiard, B., Schulze-Specking, A., Pfanner, N., and Chacinska, A. (2007). Biogenesis of the essential Tim9-Tim10 chaperone complex of mitochondria: site-specific recognition of cysteine residues by the intermembrane space receptor Mia40. J. Biol. Chem. 282, 22472-22480.

Milenkovic, D., Ramming, T., Müller, J.M., Wenz, L.-S., Gebert, N., Schulze-Specking, A., Stojanovski, D., Rospert, S., and Chacinska, A. (2009). Identification of the signal directing Tim9 and Tim 10 into the intermembrane space of mitochondria. Mol. Biol. Cell $20,2530-2539$.

Miller, B.R., and Cumsky, M.G. (1993). Intramitochondrial sorting of the precursor to yeast cytochrome c oxidase subunit Va. J. Cell Biol. 121, 1021-1029.

Moczko, M., Bömer, U., Kübrich, M., Zufall, N., Hönlinger, A., and Pfanner, N. (1997). The intermembrane space domain of mitochondrial Tom 22 functions as a trans binding site for preproteins with N-terminal targeting sequences. Mol. Cell. Biol. 17, 6574-6584. 
Model, K., Meisinger, C., and Kühlbrandt, W. (2008). Cryo-electron microscopy structure of a yeast mitochondrial preprotein translocase. J. Mol. Biol. 383, 1049-1057.

Mokranjac, D., Berg, A., Adam, A., Neupert, W., and Hell, K. (2007). Association of the Tim14.Tim16 subcomplex with the TIM23 translocase is crucial for function of the mitochondrial protein import motor. J. Biol. Chem. 282, 18037-18045.

Mokranjac, D., Bourenkov, G., Hell, K., Neupert, W., and Groll, M. (2006). Structure and function of Tim14 and Tim16, the J and J-like components of the mitochondrial protein import motor. EMBO J. 25, 4675-4685.

Mokranjac, D., Popov-Celeketić, D., Hell, K., and Neupert, W. (2005). Role of Tim21 in mitochondrial translocation contact sites. J. Biol. Chem. 280, 23437-23440.

Mokranjac, D., Sichting, M., Neupert, W., and Hell, K. (2003). Tim14, a novel key component of the import motor of the TIM23 protein translocase of mitochondria. EMBO J. 22, 4945-4956.

Mokranjac, D., Sichting, M., Popov-Celeketić, D., Mapa, K., Gevorkyan-Airapetov, L., Zohary, K., Hell, K., Azem, A., and Neupert, W. (2009). Role of Tim50 in the transfer of precursor proteins from the outer to the inner membrane of mitochondria. Mol. Biol. Cell $20,1400-1407$.

Müller, J.M., Milenkovic, D., Guiard, B., Pfanner, N., and Chacinska, A. (2008). Precursor oxidation by Mia40 and Erv1 promotes vectorial transport of proteins into the mitochondrial intermembrane space. Mol. Biol. Cell 19, 226-236.

Naoé, M., Ohwa, Y., Ishikawa, D., Ohshima, C., Nishikawa, S.I., Yamamoto, H., and Endo, T. (2004). Identification of Tim40 that mediates protein sorting to the mitochondrial intermembrane space. J. Biol. Chem. 279, 47815-47821.

Neuhoff, V., Arold, N., Taube, D., and Ehrhardt, W. (1988). Improved staining of proteins in polyacrylamide gels including isoelectric focusing gels with clear background at nanogram sensitivity using Coomassie Brilliant Blue G-250 and R-250. Electrophoresis 9, $255-262$.

Neupert, W., and Herrmann, J.M. (2007). Translocation of Proteins into Mitochondria. Annu. Rev. Biochem. 76, 723-749.

Palade, G.E. (1964). The Organization of Living Matter. Proc. Natl. Acad. Sci. USA 52, 613-634.

Papa, S., Martino, P.L., Capitanio, G., Gaballo, A., De Rasmo, D., Signorile, A., and Petruzzella, V. (2012). The oxidative phosphorylation system in mammalian mitochondria. Adv. Exp. Med. Biol. 942, 3-37.

Papic, D., Krumpe, K., Dukanovic, J., Dimmer, K.S., and Rapaport, D. (2011). Multispan mitochondrial outer membrane protein Ugo1 follows a unique Mim1-dependent import pathway. J. Cell Biol. 194, 397-405. 
Pfanner, N., Tropschug, M., and Neupert, W. (1987). Mitochondrial protein import: nucleoside triphosphates are involved in conferring import-competence to precursors. Cell $49,815-823$.

Pfanner, N., Wiedemann, N., Meisinger, C., and Lithgow, T. (2004). Assembling the mitochondrial outer membrane. Nat. Struct. Mol. Biol. 11, 1044-1048.

Pon, L., Moll, T., Vestweber, D., Marshallsay, B., and Schatz, G. (1989). Protein import into mitochondria: ATP-dependent protein translocation activity in a submitochondrial fraction enriched in membrane contact sites and specific proteins. J. Cell Biol. 109, 26032616.

Popov-Celeketić, D., Mapa, K., Neupert, W., and Mokranjac, D. (2008a). Active remodelling of the TIM23 complex during translocation of preproteins into mitochondria. EMBO J. 27, 1469-1480.

Popov-Celeketić, J., Waizenegger, T., and Rapaport, D. (2008b). Mim1 functions in an oligomeric form to facilitate the integration of Tom 20 into the mitochondrial outer membrane. J. Mol. Biol. 376, 671-680.

Popov-Čeleketić, D., Waegemann, K., Mapa, K., Neupert, W., and Mokranjac, D. (2011). Role of the import motor in insertion of transmembrane segments by the mitochondrial TIM23 complex. EMBO Rep. 12, 542-548.

Qian, X., Gebert, M., Höpker, J., Yan, M., Li, J., Wiedemann, N., van der Laan, M., Pfanner, N., and Sha, B. (2011). Structural basis for the function of Tim50 in the mitochondrial presequence translocase. J. Mol. Biol. 411, 513-519.

Qiu, J., Wenz, L.-S., Zerbes, R.M., Oeljeklaus, S., Bohnert, M., Stroud, D.A., Wirth, C., Ellenrieder, L., Thornton, N., Kutik, S., et al. (2013). Coupling of Mitochondrial Import and Export Translocases by Receptor-Mediated Supercomplex Formation. Cell 154, 596608 .

Rahman, B., Kawano, S., Yunoki-Esaki, K., Anzai, T., and Endo, T. (2014). NMR analyses on the interactions of the yeast Tim50 C-terminal region with the presequence and Tim50 core domain. FEBS Lett. 588, 678-684.

Rapaport, D., Künkele, K.P., Dembowski, M., Ahting, U., Nargang, F.E., Neupert, W., and Lill, R. (1998a). Dynamics of the TOM complex of mitochondria during binding and translocation of preproteins. Mol. Cell. Biol. 18, 5256-5262.

Rapaport, D., Mayer, A., Neupert, W., and Lill, R. (1998b). cis and trans sites of the TOM complex of mitochondria in unfolding and initial translocation of preproteins. J. Biol. Chem. 273, 8806-8813.

Rapaport, D., Neupert, W., and Lill, R. (1997). Mitochondrial protein import. Tom40 plays a major role in targeting and translocation of preproteins by forming a specific binding site for the presequence. J. Biol. Chem. 272, 18725-18731. 
Rassow, J., Guiard, B., Wienhues, U., Herzog, V., Hartl, F.U., and Neupert, W. (1989). Translocation arrest by reversible folding of a precursor protein imported into mitochondria. A means to quantitate translocation contact sites. J. Cell Biol. 109, 14211428.

Reddehase, S., Grumbt, B., Neupert, W., and Hell, K. (2009). The disulfide relay system of mitochondria is required for the biogenesis of mitochondrial Ces1 and Sod1. J. Mol. Biol. $385,331-338$.

Rehling, P., Model, K., Brandner, K., Kovermann, P., Sickmann, A., Meyer, H.E., Kühlbrandt, W., Wagner, R., Truscott, K.N., and Pfanner, N. (2003). Protein insertion into the mitochondrial inner membrane by a twin-pore translocase. Science 299, 1747-1751.

Reinhold, R., Krüger, V., Meinecke, M., Schulz, C., Schmidt, B., Grunau, S.D., Guiard, B., Wiedemann, N., van der Laan, M., Wagner, R., et al. (2012). The channel-forming Sym1 protein is transported by the TIM23 complex in a presequence-independent manner. Mol. Cell. Biol. 32, 5009-5021.

Rodríguez-Castañeda, F., Maestre-Martínez, M., Coudevylle, N., Dimova, K., Junge, H., Lipstein, N., Lee, D., Becker, S., Brose, N., Jahn, O., et al. (2010). Modular architecture of Munc13/calmodulin complexes: dual regulation by $\mathrm{Ca}^{2+}$ and possible function in shortterm synaptic plasticity. EMBO J. 29, 680-691.

Ruprecht, J.J., Hellawell, A.M., Harding, M., Crichton, P.G., McCoy, A.J., and Kunji, E.R.S. (2014). Structures of yeast mitochondrial ADP/ATP carriers support a domainbased alternating-access transport mechanism. Proc. Natl. Acad. Sci. USA 111, E426E434.

Ryan, M.T., Müller, H., and Pfanner, N. (1999). Functional staging of ADP/ATP carrier translocation across the outer mitochondrial membrane. J. Biol. Chem. 274, 20619-20627.

Saddar, S., Dienhart, M.K., and Stuart, R.A. (2008). The F1F0-ATP synthase complex influences the assembly state of the cytochrome bc1-cytochrome oxidase supercomplex and its association with the TIM23 machinery. J. Biol. Chem. 283, 6677-6686.

Saitoh, T., Igura, M., Obita, T., Ose, T., Kojima, R., Maenaka, K., Endo, T., and Kohda, D. (2007). Tom 20 recognizes mitochondrial presequences through dynamic equilibrium among multiple bound states. EMBO J. 26, 4777-4787.

Schäfer, E., Seelert, H., Reifschneider, N.H., Krause, F., Dencher, N.A., and Vonck, J. (2006). Architecture of active mammalian respiratory chain supercomplexes. J. Biol. Chem. 281, 15370-15375.

Schägger, H., and Jagow, von, G. (1991). Blue native electrophoresis for isolation of membrane protein complexes in enzymatically active form. Anal. Biochem. 199, 223-231.

Schägger, H., and Pfeiffer, K. (2000). Supercomplexes in the respiratory chains of yeast and mammalian mitochondria. EMBO J. 19, 1777-1783.

Schiller, D. (2009). Pam17 and Tim44 act sequentially in protein import into the mitochondrial matrix. Int. J. Biochem. Cell Biol. 41, 2343-2349. 
Schleyer, M., and Neupert, W. (1985). Transport of proteins into mitochondria: translocational intermediates spanning contact sites between outer and inner membranes. Cell 43, 339-350.

Schlossmann, J., Lill, R., Neupert, W., and Court, D.A. (1996). Tom71, a novel homologue of the mitochondrial preprotein receptor Tom70. J. Biol. Chem. 271, 17890-17895.

Schmidt, O., Harbauer, A.B., Rao, S., Eyrich, B., Zahedi, R.P., Stojanovski, D., Schönfisch, B., Guiard, B., Sickmann, A., Pfanner, N., Meisinger C. (2011). Regulation of Mitochondrial Protein Import by Cytosolic Kinases. Cell 144, 227-239.

Schmitt, S., Ahting, U., Eichacker, L., Granvogl, B., Go, N.E., Nargang, F.E., Neupert, W., and Nussberger, S. (2005). Role of Tom5 in maintaining the structural stability of the TOM complex of mitochondria. J. Biol. Chem. 280, 14499-14506.

Schneider, H.C., Berthold, J., Bauer, M.F., Dietmeier, K., Guiard, B., Brunner, M., and Neupert, W. (1994). Mitochondrial Hsp70/MIM44 complex facilitates protein import. Nature 371, 768-774.

Schneider, H.C., Westermann, B., Neupert, W., and Brunner, M. (1996). The nucleotide exchange factor MGE exerts a key function in the ATP-dependent cycle of mt-Hsp70Tim44 interaction driving mitochondrial protein import. EMBO J. 15, 5796-5803.

Schulz, C., Lytovchenko, O., Melin, J., Chacinska, A., Guiard, B., Neumann, P., Ficner, R., Jahn, O., Schmidt, B., and Rehling, P. (2011). Tim50's presequence receptor domain is essential for signal driven transport across the TIM23 complex. J. Cell Biol. 195, 643-656.

Schülke, N., Sepuri, N.B., and Pain, D. (1997). In vivo zippering of inner and outer mitochondrial membranes by a stable translocation intermediate. Proc. Natl. Acad. Sci. USA 94, 7314-7319.

Schwaiger, M., Herzog, V., and Neupert, W. (1987). Characterization of translocation contact sites involved in the import of mitochondrial proteins. J. Cell Biol. 105, 235-246.

Setoguchi, K., Otera, H., and Mihara, K. (2006). Cytosolic factor- and TOM-independent import of C-tail-anchored mitochondrial outer membrane proteins. EMBO J. 25, 56355647.

Sherman, E.L., Taylor, R.D., Go, N.E., and Nargang, F.E. (2006). Effect of Mutations in Tom40 on Stability of the Translocase of the Outer Mitochondrial Membrane (TOM) Complex, Assembly of Tom40, and Import of Mitochondrial Preproteins. J. Biol. Chem. 281, 22554-22565.

Sherman, E.L., Go, N.E., and Nargang, F.E. (2005). Functions of the small proteins in the TOM complex of Neurospora crasssa. Mol. Biol. Cell 16, 4172-4182.

Shiota, T., Mabuchi, H., Tanaka-Yamano, S., Yamano, K., and Endo, T. (2011). In vivo protein-interaction mapping of a mitochondrial translocator protein Tom 22 at work. Proc. Natl. Acad. Sci. USA 108, 15179-15183. 
Sideris, D.P., Petrakis, N., Katrakili, N., Mikropoulou, D., Gallo, A., Ciofi-Baffoni, S., Banci, L., Bertini, I., and Tokatlidis, K. (2009). A novel intermembrane space-targeting signal docks cysteines onto Mia40 during mitochondrial oxidative folding. J. Cell. Biol. 187, 1007-1022.

Sikorski, R.S., and Hieter, P. (1989). A system of shuttle vectors and yeast host strains designed for efficient manipulation of DNA in Saccharomyces cerevisiae. Genetics 122, $19-27$.

Sirrenberg, C., Endres, M., Becker, K., Bauer, M.F., Walther, E., Neupert, W., and Brunner, M. (1997). Functional cooperation and stoichiometry of protein translocases of the outer and inner membranes of mitochondria. J. Biol. Chem. 272, 29963-29966.

Sirrenberg, C., Endres, M., Fölsch, H., Stuart, R.A., Neupert, W., and Brunner, M. (1998). Carrier protein import into mitochondria mediated by the intermembrane proteins Tim10/Mrs11 and Tim12/Mrs5. Nature 391, 912-915.

Soding, J., Biegert, A., and Lupas, A.N. (2005). The HHpred interactive server for protein homology detection and structure prediction. Nucleic Acids Res. 33, W244-W248.

Stan, T., Ahting, U., Dembowski, M., Künkele, K.P., Nussberger, S., Neupert, W., and Rapaport, D. (2000). Recognition of preproteins by the isolated TOM complex of mitochondria. EMBO J. 19, 4895-4902.

Steger, H.F., Söllner, T., Kiebler, M., Dietmeier, K.A., Pfaller, R., Trülzsch, K.S., Tropschug, M., Neupert, W., and Pfanner, N. (1990). Import of ADP/ATP carrier into mitochondria: two receptors act in parallel. J. Cell Biol. 111, 2353-2363.

Stojanovski, D., Guiard, B., Kozjak-Pavlovic, V., Pfanner, N., and Meisinger, C. (2007). Alternative function for the mitochondrial SAM complex in biogenesis of alpha-helical TOM proteins. J. Cell Biol. 179, 881-893.

Stojanovski, D., Milenkovic, D., Müller, J.M., Gabriel, K., Schulze-Specking, A., Baker, M.J., Ryan, M.T., Guiard, B., Pfanner, N., and Chacinska, A. (2008). Mitochondrial protein import: precursor oxidation in a ternary complex with disulfide carrier and sulfhydryl oxidase. J. Cell Biol. 183, 195-202.

Stroud, D.A., Becker, T., Qiu, J., Stojanovski, D., Pfannschmidt, S., Wirth, C., Hunte, C., Guiard, B., Meisinger, C., Pfanner, N., et al. (2011). Biogenesis of mitochondrial $\beta$-barrel proteins: the POTRA domain is involved in precursor release from the SAM complex. Mol. Biol. Cell 22, 2823-2833.

Stuart, R.A. (2008). Supercomplex organization of the oxidative phosphorylation enzymes in yeast mitochondria. J. Bioenerg. Biomembr. 40, 411-417.

Suzuki, H., Kadowaki, T., Maeda, M., Sasaki, H., Nabekura, J., Sakaguchi, M., and Mihara, K. (2004). Membrane-embedded C-terminal segment of rat mitochondrial TOM40 constitutes protein-conducting pore with enriched beta-structure. J. Biol. Chem. 279, 50619-50629. 
Tamura, Y., Harada, Y., Shiota, T., Yamano, K., Watanabe, K., Yokota, M., Yamamoto, H., Sesaki, H., and Endo, T. (2009). Tim23-Tim50 pair coordinates functions of translocators and motor proteins in mitochondrial protein import. J. Cell Biol. 184, 129141.

Tamura, Y., Harada, Y., Yamano, K., Watanabe, K., Ishikawa, D., Ohshima, C., Nishikawa, S.-I., Yamamoto, H., and Endo, T. (2006). Identification of Tam41 maintaining integrity of the TIM23 protein translocator complex in mitochondria. J. Cell Biol. 174, 631-637.

Terziyska, N., Grumbt, B., Kozany, C., and Hell, K. (2009). Structural and functional roles of the conserved cysteine residues of the redox-regulated import receptor Mia40 in the intermembrane space of mitochondria. J. Biol. Chem. 284, 1353-1363.

Thornton, N., Stroud, D.A., Milenkovic, D., Guiard, B., Pfanner, N., and Becker, T. (2010). Two modular forms of the mitochondrial sorting and assembly machinery are involved in biogenesis of alpha-helical outer membrane proteins. J. Mol. Biol. 396, 540 549.

Truscott, K.N., Kovermann, P., Geissler, A., Merlin, A., Meijer, M., Driessen, A.J., Rassow, J., Pfanner, N., and Wagner, R. (2001). A presequence- and voltage-sensitive channel of the mitochondrial preprotein translocase formed by Tim23. Nat. Struct. Biol. 8 , 1074-1082.

Truscott, K.N., Voos, W., Frazier, A.E., Lind, M., Li, Y., Geissler, A., Dudek, J., Müller, H., Sickmann, A., Meyer, H.E., et al. (2003). A J-protein is an essential subunit of the presequence translocase-associated protein import motor of mitochondria. J. Cell Biol. $163,707-713$.

Truscott, K.N., Wiedemann, N., Rehling, P., Müller, H., Meisinger, C., Pfanner, N., and Guiard, B. (2002). Mitochondrial import of the ADP/ATP carrier: the essential TIM complex of the intermembrane space is required for precursor release from the TOM complex. Mol. Cell. Biol. 22, 7780-7789.

Ujwal, R., Cascio, D., Colletier, J.-P., Faham, S., Zhang, J., Toro, L., Ping, P., and Abramson, J. (2008). The crystal structure of mouse VDAC1 at $2.3 \AA$ resolution reveals mechanistic insights into metabolite gating. Proc. Natl. Acad. Sci. USA 105, 1774217747.

Ungermann, C., Neupert, W., and Cyr, D.M. (1994). The role of Hsp70 in conferring unidirectionality on protein translocation into mitochondria. Science $266,1250-1253$.

van der Laan, M., Urbanus, M.L., Hagen-Jongman, Ten, C.M., Nouwen, N., Oudega, B., Harms, N., Driessen, A.J.M., and Luirink, J. (2003). A conserved function of YidC in the biogenesis of respiratory chain complexes. Proc. Natl. Acad. Sci. USA 100, 5801-5806.

van der Laan, M., Chacinska, A., Lind, M., Perschil, I., Sickmann, A., Meyer, H.E., Guiard, B., Meisinger, C., Pfanner, N., and Rehling, P. (2005). Pam17 is required for architecture and translocation activity of the mitochondrial protein import motor. Mol. Cell. Biol. 25, 7449-7458. 
van der Laan, M., Hutu, D.P., and Rehling, P. (2010). On the mechanism of preprotein import by the mitochondrial presequence translocase. Biochim. Biophys. Acta 1803, 732739.

van der Laan, M., Meinecke, M., Dudek, J., Hutu, D.P., Lind, M., Perschil, I., Guiard, B., Wagner, R., Pfanner, N., and Rehling, P. (2007). Motor-free mitochondrial presequence translocase drives membrane integration of preproteins. Nat. Cell Biol. 9, 1152-1159.

van der Laan, M., Wiedemann, N., Mick, D.U., Guiard, B., Rehling, P., and Pfanner, N. (2006). A role for Tim 21 in membrane-potential-dependent preprotein sorting in mitochondria. Curr. Biol. 16, 2271-2276.

van Wilpe, S., Ryan, M.T., Hill, K., Maarse, A.C., Meisinger, C., Brix, J., Dekker, P.J., Moczko, M., Wagner, R., Meijer, M., et al. (1999). Tom22 is a multifunctional organizer of the mitochondrial preprotein translocase. Nature 401, 485-489.

Vestweber, D., Brunner, J., Baker, A., and Schatz, G. (1989). A 42K outer-membrane protein is a component of the yeast mitochondrial protein import site. Nature 341, 205209.

Voisine, C., Craig, E.A., Zufall, N., Ahsen, von, O., Pfanner, N., and Voos, W. (1999). The protein import motor of mitochondria: unfolding and trapping of preproteins are distinct and separable functions of matrix Hsp70. Cell 97, 565-574.

Vögtle, F.-N., Burkhart, J.M., Rao, S., Gerbeth, C., Hinrichs, J., Martinou, J.-C., Chacinska, A., Sickmann, A., Zahedi, R.P., and Meisinger, C. (2012). Intermembrane space proteome of yeast mitochondria. Mol. Cell Proteomics 11, 1840-1852.

Vögtle, F.-N., Wortelkamp, S., Zahedi, R.P., Becker, D., Leidhold, C., Gevaert, K., Kellermann, J., Voos, W., Sickmann, A., Pfanner, N., et al. (2009). Global analysis of the mitochondrial N-proteome identifies a processing peptidase critical for protein stability. Cell 139, 428-439.

Wagner, K., Gebert, N., Guiard, B., Brandner, K., Truscott, K.N., Wiedemann, N., Pfanner, N., and Rehling, P. (2008). The assembly pathway of the mitochondrial carrier translocase involves four preprotein translocases. Mol. Cell. Biol. 28, 4251-4260.

Waizenegger, T., Habib, S.J., Lech, M., Mokranjac, D., Paschen, S.A., Hell, K., Neupert, W., and Rapaport, D. (2004). Tob38, a novel essential component in the biogenesis of beta-barrel proteins of mitochondria. EMBO Rep. 5, 704-709.

Walsh, P., Bursać, D., Law, Y.C., Cyr, D., and Lithgow, T. (2004). The J-protein family: modulating protein assembly, disassembly and translocation. EMBO Rep. 5, 567-571.

Webb, C.T., Gorman, M.A., Lazarou, M., Ryan, M.T., and Gulbis, J.M. (2006). Crystal Structure of the Mitochondrial Chaperone TIM9•10 Reveals a Six-Bladed $\alpha$-Propeller. Mol. Cell 21, 123-133.

Wideman, J.G., Lackey, S.W.K., Srayko, M.A., Norton, K.A., and Nargang, F.E. (2013). Analysis of mutations in Neurospora crassa ERMES components reveals specific functions related to $\beta$-barrel protein assembly and maintenance of mitochondrial morphology. PLoS ONE 8, e71837. 
Wiedemann, N., Pfanner, N., and Ryan, M.T. (2001). The three modules of ADP/ATP carrier cooperate in receptor recruitment and translocation into mitochondria. EMBO J. 20, 951-960.

Wiedemann, N., Frazier, A.E., and Pfanner, N. (2004a). The protein import machinery of mitochondria. J. Biol. Chem. 279, 14473-14476.

Wiedemann, N., Kozjak, V., Chacinska, A., Schönfisch, B., Rospert, S., Ryan, M.T., Pfanner, N., and Meisinger, C. (2003). Machinery for protein sorting and assembly in the mitochondrial outer membrane. Nature 424, 565-571.

Wiedemann, N., Pfanner, N., and Rehling, P. (2006). Import of precursor proteins into isolated yeast mitochondria. Methods Mol. Biol. 313, 373-383.

Wiedemann, N., Truscott, K.N., Pfannschmidt, S., Guiard, B., Meisinger, C., and Pfanner, N. (2004b). Biogenesis of the protein import channel Tom40 of the mitochondrial outer membrane: intermembrane space components are involved in an early stage of the assembly pathway. J. Biol. Chem. 279, 18188-18194.

Wiedemann, N., van der Laan, M., Hutu, D.P., Rehling, P., and Pfanner, N. (2007). Sorting switch of mitochondrial presequence translocase involves coupling of motor module to respiratory chain. J. Cell Biol. 179, 1115-1122.

Wimley, W.C. (2003). The versatile beta-barrel membrane protein. Curr. Opin. Struct. Biol. 13, 404-411.

Winge, D.R. (2012). Sealing the mitochondrial respirasome. Mol. Cell. Biol. 32, 26472652.

Wittelsberger, A., Thomas, B.E., Mierke, D.F., and Rosenblatt, M. (2006). Methionine acts as a "magnet" in photoaffinity crosslinking experiments. FEBS Lett. 580, 1872-1876.

Wrobel, L., Trojanowska, A., Sztolsztener, M.E., and Chacinska, A. (2013). Mitochondrial protein import: Mia40 facilitates Tim22 translocation into the inner membrane of mitochondria. Mol. Biol. Cell 24, 543-554.

Yamano, K., Yatsukawa, Y.-I., Esaki, M., Hobbs, A.E.A., Jensen, R.E., and Endo, T. (2008). Tom 20 and Tom 22 share the common signal recognition pathway in mitochondrial protein import. J. Biol. Chem. 283, 3799-3807.

Young, J.C., Hoogenraad, N.J., and Hartl, F.U. (2003). Molecular chaperones Hsp90 and Hsp70 deliver preproteins to the mitochondrial import receptor Tom70. Cell 112, 41-50.

Youngman, M.J., Hobbs, A.E.A., Burgess, S.M., Srinivasan, M., and Jensen, R.E. (2004). $\mathrm{Mmm} 2 \mathrm{p}$, a mitochondrial outer membrane protein required for yeast mitochondrial shape and maintenance of mtDNA nucleoids. J. Cell Biol. 164, 677-688.

Zara, V., Ferramosca, A., Robitaille-Foucher, P., Palmieri, F., and Young, J.C. (2009). Mitochondrial carrier protein biogenesis: role of the chaperones Hsc70 and Hsp90.

Biochem. J. 419, 369-375. 
Zimmermann, R., and Neupert, W. (1980). Transport of proteins into mitochondria. Posttranslational transfer of ADP/ATP carrier into mitochondria in vitro. Eur. J. Biochem. 109, 217-229. 


\section{Acknowledgments}

First and foremost, I would like to thank my supervisor Prof. Dr. Peter Rehling for going above and beyond what was expected, enabling me to work on fascinating projects and counseling me along every step of the way. His close guidance throughout my years in his lab made researching an enjoyable and intellectually rewarding experience.

To my thesis committee members, Prof. Dr. Ivo Feussner and Prof. Dr. Christian Griesinger, thank you for making my committee meetings a pleasant experience and for sharing with me your valuable insight.

To Dr. Christian Schulz, thank you for passing on a great deal of technical and intellectual knowledge. A significant portion of this work would not have been possible if it was not for your excellent guidance.

Dr. Oleksandr Lytovchenko first introduced me to work in a devoted protein biochemistry lab and through his kind and patient manner, he instilled upon me a fondness for this new field. To him, I am especially grateful for the many years of fruitful collaborative research.

The past and present members of the Rehling lab have made my years as a $\mathrm{PhD}$ student an enjoyable experience, and to them I am grateful.

The excellent management of the IMPRS by Steffen Burkhardt and Kerstin Grüniger made my academic transition to Germany a trouble-free and pleasurable experience, thank you for all of your assistance.

Last, but certainly not least, I would like to express my deepest gratitude to my parents and my incredibly supportive wife: without their continued support my academic goals would have never been reached! 


\title{
Jonathan R. Melin
}

\author{
PERSONAL INFORMATION
}

\section{Date of birth \\ Family status Citizenship}

\author{
July $3^{\text {rd }}, 1986$ in Memphis, TN, USA \\ Married, no children \\ USA and Germany
}

\begin{abstract}
EDUCATION
University of Göttingen Medical School, Göttingen, Germany. Doctor of Natural Sciences (PhD) Sept 2010-June 2014

International Max Planck Research School, Göttingen, Germany. Masters of Science

Sept 2009-Aug 2010

University of California, Irvine, Irvine, CA, USA. Bachelor of Science in Biology

Sept 2005-June 2009

King's College London, London, United Kingdom.

Sept 2007-June 2008

Westlake High School, Thousand Oaks, CA, USA.

Sept 2001-June 2005
\end{abstract}

\section{PUBLICATIONS}

Melin J., Schulz C., Wrobel L., Bernhard O., Chacinska A., Jahn O., Schmidt B., Rehling P. "Presequence recognition by the Tom40 channel promotes precursor translocation towards the inner membrane translocase." In revision.

Lytovchenko O., Melin J., Schulz C., Kilisch M., Hutu D.P., and Rehling P. "Signal recognition initiates reorganization of the presequence translocase during protein import." EMBO J. Mar. 2013: 32(6): 886-98.

Schulz C., Lytovchenko O., Melin J., Chacinska A., Guiard B., Neumann P., Ficner R., Jahn O., Schmidt B. and Rehling P. "Tim50's presequence receptor domain is essential for signal driven transport across the TIM23 complex." J. Cell Biol. Nov. 2011: 195(4): 643-56.

Furst D.E., Saag K.G. and Melin J. "Strategies for treating rheumatoid arthritis: new guidelines from the ACR." Int. J. Clin. Rheumatol. Feb. 2009: 4(1): 3-5.

\section{AWARDS}

Excellence Stipend Recipient International Max Planck Research School

Dean's List (Biology) University of California, Irvine

John Hollowell Research Writing Award University of California, Irvine Title of Paper: The Uninsured States of America
Sept. 2009-Aug. 2010

Fall 2005-Spring 2007

Spring 2006 